NBER WORKING PAPER SERIES

\title{
DISTORTIONS IN PRODUCTION NETWORKS
}

\author{
Saki Bigio \\ Jennifer La'O \\ Working Paper 22212 \\ http://www.nber.org/papers/w22212
NATIONAL BUREAU OF ECONOMIC RESEARCH
1050 Massachusetts Avenue
Cambridge, MA 02138
April 2016

We thank Daron Acemoglu, Marios Angeletos, Enghin Atalay, David Baqaee, Vasco Carvalho, Gabriel Chodorow-Reich, Mark Gertler, Michael Gofman, Bob Hall, James Hamilton, Pat Kehoe, Pete Klenow, Ricardo Lagos, Ezra Oberfield, Valerie Ramey, Ricardo Reis, Alireza Tahbaz-Salehi, and Pierre-Daniel Sarte for their valuable feedback and suggestions. We owe special thanks to V.V. Chari, Ian Dew-Becker, Chad Jones, Marco Ortiz, and Alberto Trejos for their insightful conference discussions of this paper. Finally, we have benefited greatly from the comments received in numerous conferences and seminars. The views expressed herein are those of the authors and do not necessarily reflect the views of the National Bureau of Economic Research.

NBER working papers are circulated for discussion and comment purposes. They have not been peerreviewed or been subject to the review by the NBER Board of Directors that accompanies official NBER publications.

(C) 2016 by Saki Bigio and Jennifer La'O. All rights reserved. Short sections of text, not to exceed two paragraphs, may be quoted without explicit permission provided that full credit, including @ notice, is given to the source. 
Distortions in Production Networks

Saki Bigio and Jennifer La'O

NBER Working Paper No. 22212

April 2016, Revised August 2019

JEL No. C67,E32,E42,G01,G10

\begin{abstract}
$\underline{\text { ABSTRACT }}$
How does an economy's production structure determine its macroeconomic response to sectoral distortions? We study a static framework in which production is organized in an input-output network and firms' production decisions are distorted. We show how sectoral distortions manifest at the aggregate level via two channels: total factor productivity and the labor wedge. The strength of each channel depends jointly on the input-output structure and the distribution of shocks. Near efficiency, distortions generate zero first-order effects on TFP but non-zero first-order effects on the labor wedge; the latter we show to be determined by the sector's network "centrality." We apply the model to the 2008-09 Financial Crisis and find that the U.S. input-ouput network may have amplified financial distortions by roughly a factor of two relative to a counterfactual economy devoid of intermediate good trade.
\end{abstract}

\title{
Saki Bigio
}

Department of Economics

University of California at Los Angeles

8283 Bunche Hall

Box 951477

Los Angeles, CA 90095-1477

and NBER

sbigio@econ.ucla.edu

Jennifer La'O

Columbia University

Department of Economics

1105A International Affairs Building

420 West 118th Street

New York, NY 10027

and NBER

j14196@columbia.edu 


\section{Introduction}

A downward glance from an airplane window can often attest to the astounding complexity behind economic production. Warehouses, silos, and freight loading zones adjoin manufacturing and energy plants. A mosaic of intersecting highways, railroads, and waterways transport shipments to and from producers. By and large, the economy's gross domestic product is the outcome of an elaborate, multi-step production process involving millions of inter-firm transactions.

Actors in this system are not, however, free from disturbances. Firms face a changing array of frictions - e.g. taxes, financial constraints, and monopolistic competition - all of which actively distort individual production choices away from their optima. In a complex network of intermediate good trade, individual decisions affect not only the firms themselves but their trading partners, their trading partners' partners, and so on. The aggregate effects of such frictions depend on the overall network architecture. In this paper we examine how an economy's production structure determines its macroeconomic response to micro-level distortions.

The Framework. We build a static, multi-sector, general equilibrium model of inter-sectoral trade à la Long and Plosser (1983), and more recently Acemoglu et al. (2012). Firms operate Cobb-Douglas technologies, employ labor, demand intermediate goods, and face sectoral productivity shocks. Intermediate goods are produced by all sectors; the matrix of cross-sector input requirements constitutes the input-output network. A representative household consumes final goods and supplies labor elastically.

Within this framework, we allow sectoral production choices to be distorted. Distortions are captured by wedges between firms' marginal revenue and marginal costs. While we remain agnostic regarding the microeconomic origin of these distortions, we show how they may easily be rationalized by tax rates, mark-ups, financial constraints, and the like. Regardless of their underlying source, however, distortions result in excess revenue over costs, e.g. in the form of profits or tax revenue. We allow for two outcomes of this excess revenue: either they are rebated lump-sum to the household or they are completely destroyed - thrown into the ocean.

Firms in our model are thereby subject to three classes of sectoral shocks: (i) productivity shocks, (ii) distortions whose proceeds are thrown out, what we refer to as "wasted distortions," and (iii) distortions whose proceeds are rebated lump-sum to the household, or "rebated distortions." For arbitrary network structures and distributions of these shocks, we characterize the equilibrium of the economy and derive the following three main results.

A Macroeconomic Decomposition. Our first main result is a decomposition of the macroeconomic effects of sectoral distortions into two distinct channels: movement in total factor produc- 
tivity (TFP) and movement in the labor wedge. Specifically, we show that the economy admits an aggregate representation à la Chari et al. (2007) in which the equilibrium may be characterized by two equations: an aggregate production function and an intratemporal condition. The production function admits a TFP component that is a function of the input-output network and all three types of sectoral shocks. The intratemporal condition admits a wedge between the household's marginal rate of substitution between consumption and labor and the economy's marginal rate of transformation. Following the literature, we call this wedge the labor wedge and we show that it is a non-linear function of the underlying input-output network and the rebated distortions alone.

Our decomposition of the aggregate effects of sectoral shocks into these two channels highlights the disparity by which the three types of shocks - sectoral productivities, wasted distortions, and rebated distortions - manifest at the aggregate level. In terms of equilibrium allocations, wasted distortions are isomorphic to productivity shocks: they lead to a true reduction of output per worker. As a result, these shocks operate through TFP alone.

Rebated distortions, on the other hand, distort the choices of firms but do not actively destroy resources. Instead, these shocks simply move sectors away from their optimal scale, resulting in an inefficient reallocation of inputs across the network. This reduction in aggregate productive efficiency manifests as a fall in TFP. Furthermore, as distorted firm choices aggregate within the production network, they ultimately drive a wedge between the economy's marginal product of labor and the real wage, thereby generating an aggregate labor wedge.

The First-Order Effects of Distortions. Following this decomposition, we examine the firstorder effects of rebated distortions. Our second main result is the following: near the efficient benchmark, rebated distortions have zero first-order effects on TFP but non-zero first-order effects on the aggregate labor wedge.

Rebated distortions reduce the productive efficiency of the economy via misallocation. Regardless of the network structure, misallocation generates a fall in TFP through second- and higherorder terms alone. This is in stark contrast to the aggregate effects of wasted distortions which have non-zero first-order effects on TFP globally. This suggests that the modeling of the payments of distortions is crucial for inferring their macroeconomic implications.

Sectoral rebated distortions do, however, have first-order effects on the aggregate labor wedge. We show that these effects may be succinctly characterized by a vector of constants signifying each sectors' network "centrality."

To understand this result, first consider the aggregate effects of sectoral productivity shocks in multi-sector Cobb-Douglas input-output economies, as studied by Long and Plosser (1983); Gabaix (2011); Acemoglu et al. (2012). In this class of economies, it is well-known that the aggregate effects of sectoral productivities may be neatly summarized by a vector of constants determined by the 
Leontief inverse matrix, see Burress (1994). The Leontief inverse matrix captures the entire infinite sequence of all higher-order network effects; in fact, its mathematical structure is closely related to the concept of Bonacich centrality. From this we infer that sectors with higher "centrality" in the network - sectors with more direct and indirect downstream customers - exhibit greater influence on TFP than sectors with lower centrality. Acemoglu et al. (2012) term this vector the "influence vector," but to distinguish across the different shocks and the two aggregate channels in our model, we instead refer more specifically to this vector as the "TFP influence of productivities."

Now consider sectoral distortions. We show that within this class of economies, the first-order effects of small rebated distortions on the aggregate labor wedge are captured by a vector of nonzero constants. This vector, which we term the "labor wedge influence of distortions," we show to be approximately equal to the TFP influence of productivities. A major implication of this result is that the "centrality" of a sector - as indicated by the Leontief inverse matrix - determines not only the contribution of the sector's productivity shock to TFP but also, to a first-order approximation, the contribution of the sector's distortion to the aggregate labor wedge.

Finally, our third main result regards the measurement of the labor wedge influence vector. We show that sufficient statistics for the elements of this vector are the Domar weights: the ratio of sectoral sales to GDP. It is a well-known result that the Domar weights are sufficient statistics for the first-order effects of sectoral productivities on TFP; this is the celebrated Hulten's theorem, Hulten (1978). Given that the first-order effects of rebated distortions on the aggregate labor wedge are equal to the first-order effects of productivities on TFP, and combining this with the fact that the Domar weights are sufficient statistics for the latter, we infer that they are also sufficient statistics for the former.

Simple Network Examples. While the centrality results for the general network economy may be helpful for intuition, one must keep in mind that they hold only up to to a first-order approximation around efficiency. To the extent that the economy is away from efficiency, the labor wedge influence of distortions depends jointly on the network structure and the entire distribution of the shocks. In this case, the simple notion of network centrality is no more sufficient.

We thereby consider a few simple network economies. We use these to demonstrate how different network structures result in different ways by which sectoral distortions manifest in TFP and the labor wedge. The general lesson from these examples is that sectors can differ substantially in their influence across the two aggregate channels: distortions in certain key "bottleneck" sectors can have disproportionately large effects on the aggregate labor wedge but at the same time contribute the least to movements in TFP. 
A Quantitative Illustration. In the final section of the paper we consider a particular quantitative application of the model. We calibrate the production network to the US input-output tables provided by the Bureau of Economic Analysis (BEA) and evaluate the extent to which the U.S. network structure may have amplified financial frictions during the 2008-2009 Financial Crisis. We abstract from productivity shocks and focus on rebated distortions caused by financial constraints. In particular, we use panel data on sectoral credit spreads constructed and provided by Gilchrist and Zakrajšek (2012) as our proxy for financial distortions. We feed the "GZ distortion" series into our calibrated model and produce the following results.

First, we find that the model generates quantitatively larger movements in the aggregate labor wedge than in aggregate TFP over our sample period (1998-2015). Given that we abstract entirely from productivity shocks in this exercise, this result squares with the theory: small distortions have only second and higher-order effects on TFP, but first-order effects on the aggregate labor wedge. Moreover, we find that the model-implied movements in the aggregate labor wedge are surprisingly similar to those we compute in the data both in size and in correlation.

Next we focus on the years surrounding the financial crisis and ask: what is the role played by the input-output network in amplifying sectoral financial frictions during the Financial Crisis? We construct a measure we call the "labor wedge network multiplier" defined as the ratio between the percentage drop in the aggregate labor wedge generated by the calibrated network economy and that generated by an otherwise equivalent economy without input-output linkages. Our model generates a labor wedge network multiplier of roughly 2 , indicating that the U.S. input-output network may have generated considerable amplification of financial shocks during the crisis.

A certain back-of-the-envelope calculation helps one interpret this number. Our theoretical results indicate that near efficiency, a sufficient statistic for the labor wedge network multiplier is the sum of the Domar weights which itself is equal to total sales over GDP. In our data, total sales over GDP is roughly 2.

Finally, we examine the calibrated model's cross-sectional implications. We find that the calibrated input-output network can better match the observed cross-sectional correlation in sales growth during this period relative to an otherwise equivalent economy without input-output linkages. In fact, given the same GZ distortion series, the calibrated network outperforms $99.5 \%$ of random network economies in matching cross-sectoral co-movements in sales.

Related Literature. Following the canonical multi-sector model of Long and Plosser (1983), an extensive literature has studied the aggregate effects of sectoral productivity shocks. The primary focus of this literature has been the transmission of sector-specific productivity shocks via the input-output network; see Horvath (1998, 2000); Dupor (1999) and more recently, Gabaix (2011) and Acemoglu et al. (2012). Carvalho (2014) provides an extensive survey. 
Our paper likewise builds on the input-output model of Long and Plosser (1983), but rather than examine the aggregate effects of productivity shocks, we instead shift focus toward the aggregate effects of sectoral distortions. Our paper thereby belongs to an adjacent field of research on the transmission of distortions in economies with intermediate good trade. Influential work by Basu (1995), Ciccone (2002), Yi (2003), Jones (2011), and Asker et al. (2014) allow for distortions in within specific input-output structures. ${ }^{1}$ Our paper, along with the work by Jones (2013), Baqaee and Farhi (2019a), and Liu (2017), study the macroeconomic effects of distortions in economies with general input-output structures. We discuss the relationship to these three papers here but also throughout our analysis.

Our paper builds heavily on Jones (2013). In the closed-economy variant of Jones (2013), producers use both intermediate goods and primary factors - physical and human capital - in production. Jones (2013) demonstrates how sectoral distortions generate input-misallocation and how misallocation may be amplified by the economy's input-output structure. Differences in production networks may thereby help explain long run cross-country growth differentials.

As in our paper, Jones (2013) restricts the class of production functions and the household's consumption bundle to be Cobb-Douglas. In contrast, Baqaee and Farhi (2019a) allow for greater generality in production: firms operate technologies homogeneous of degree one and employ any finite number of primary factors. Baqaee and Farhi (2019a) show how even in their very general environment one may characterize the macroeconomic effects of productivity shocks and distortions. In particular, they generalize earlier work by Hulten (1978) and provide an elegant decomposition of the first-order effects of productivities and distortions on TFP both at and away from efficiency.

The primary difference between our model and those of Jones (2013) and Baqaee and Farhi (2019a) is the treatment of primary factors. While the supply of primary factors in those papers are exogenously fixed, we allow for the supply of labor - our primary factor of production - to be endogenously determined. ${ }^{2}$ This modeling choice has the following implication. With primary factors fixed, the distortions in both Jones (2013) and Baqaee and Farhi (2019a) manifest solely in total factor productivity (TFP). Jones (2013) obtains a closed-form solution for TFP in a CobbDouglas economy while Baqaee and Farhi (2019a) characterize the first-order effects of distortions on TFP both at and away from efficiency in a far more general economy. ${ }^{3}$

\footnotetext{
${ }^{1}$ Jones (2011) examines a single-intermediate input economy and focuses on different elasticities of substitution. Ciccone (2002) similarly develops a multiplier formula on the growth of an economy with intermediate good production. In the economic fluctuations literature, Basu (1995) shows that in a demand-driven business cycle model, the combination of counter-cyclical markups (distortions) and input-output production can result in pro-cyclical TFP. Finally, Yi (2003) finds that tariffs can be amplified when intermediate goods are traded through multiple stages of production.

${ }^{2}$ Jones (2013) assumes a fixed, exogenous aggregate supply of two primary factors: physical and human capital. Baqaee and Farhi (2019a), on the other hand, allow for any finite number of primary factors all in exogenous fixed supply.

${ }^{3}$ Hulten (1978) was the first to show through macro-envelope conditions that in efficient economies the first-order
} 
In contrast, we allow for the primary factor in our model, labor, to be supplied elastically by the household. Sectoral distortions thereby arise not only in TFP but also in the aggregate labor wedge. We provide a closed-form solution for the labor wedge for any arbitrary network structure and distribution of distortions within the class of multi-sector, Cobb-Douglas inputoutput economies. Our characterization of the aggregate labor wedge and all subsequent results derived from it-including its first-order approximation - are, to the best of our knowledge, novel contributions of this paper. ${ }^{4}$

Finally, in both Jones (2013) and Baqaee and Farhi (2019a), distortions are modeled as taxes in which the revenue is rebated entirely to the household, what we call "rebated" distortions. Assuming that payments from distortions are redistributed lump-sum to the household is also the approach generally taken in the misallocation literature; see e.g. Restuccia and Rogerson (2008).

Thus, a secondary difference between our paper and Jones (2013) and Baqaee and Farhi (2019a) is that in addition to these shocks, we allow for "wasted" distortions. This draws an immediate connection between our paper and that of Liu (2017). Liu (2017) similarly studies a multi-sector economy with a general input-output network, but instead focuses exclusively on distortions whose payments are discarded. He studies the effects of industrial policy: within his model he asks whether a central government should intervene and subsidize production in select sectors when they are subject to this class of shocks.

Therefore, in contrast to Jones (2013), Baqaee and Farhi (2019a), and to Liu (2017), we allow for both rebated and wasted distortions. Rather than take a stand on which class is more realistic, we characterize the aggregate effects of both and show how they have vastly different macroeconomic implications. In the context of our model we show that wasted distortions are isomorphic to productivity shocks; we thereby forge a connection between the literature on sectoral wasted distortions and the aforementioned literature on the effects of sectoral productivity shocks.

Finally, we apply our model to the 2008-2009 Financial Crisis. In this quantitative application, we interpret the distortions specifically as financial frictions generated by working capital constraints. One clear limitation of our approach is that we treat financial constraints as exogenous: we do not explicitly model how firms obtain credit. Recent papers by Altinoglu (2018), Reischer (2019), and Luo (2016) extend our framework by endogenizing the financial friction. These papers study input-output economies in which firms finance working capital through either bank or trade credit. The latter type of credit is provided by intermediate good suppliers and, as a result,

effects of firm productivity shocks on TFP are equal to the Domar weights, i.e. firm sales as a share of GDP. In Hulten (1978), all shocks were efficient. Baqaee and Farhi (2019a) generalize Hulten (1978) to equilibrium allocations away from efficiency using micro-envelope conditions. See also Baqaee and Farhi (2019b) for a characterization of the second-order effects of sectoral productivity shocks at the efficient allocation in general economies.

${ }^{4}$ Fadinger et al. (2018) also study a Cobb-Douglas input-output economy based on Jones (2013). Like Jones (2013), they too assume an exogenous primary factor supply. 
input-output linkages coincide with trade credit linkages. We provide a more detailed summary of these papers in our quantitative application section; our model is however nested in all three as the reduced-form in which working capital constraints are exogenous.

Layout. This paper is organized as follows. Section 2 introduces the framework. Section 3 defines and characterizes equilibrium allocations. Section 4 presents our key aggregation results on how sectoral distortions manifest at the macro level. Section 5 presents three simple, tractable examples that help illustrate the theoretical lessons of our paper. Section 6 provides a quantitative illustration of the model applied to the 2008-2009 Financial Crisis. Finally, Section 7 concludes. The Appendix contains all proofs and supporting material.

\section{The Environment}

The economy is static and populated by a representative household and $N$ production sectors, indexed by $i \in I=\{1, \ldots, N\}$. Each sector consists of two types of firms: (i) a unit mass of monopolistically-competitive firms, indexed by $k \in[0,1]$, producing differentiated goods and (ii) a perfectly-competitive producer whose sole purpose is to aggregate the industry's differentiated goods into a uniform sectoral good. The output of each industry can be either consumed by the household or used as an intermediate input to production. Finally there is a government which may levy sector-specific revenue taxes or subsidies and distributes part or all of the proceeds lump-sum back to the household.

Production. Technology is identical within sectors but heterogeneous across sectors. The production of firm $k$ in sector $i$ is a constant returns-to-scale Cobb-Douglas technology given by

$$
y_{i, k}=z_{i} \ell_{i, k}^{\alpha_{i}}\left(x_{i, k}\right)^{1-\alpha_{i}}
$$

where $y_{i, k}$ is the firm's output, $\ell_{i, k}$ its labor, and $x_{i, k}$ is a composite of the firm's intermediate inputs. The parameter $\alpha_{i} \in[0,1]$ denotes the firm's labor share and $z_{i}$ is a sector-specific productivity shock. The firm's intermediate goods basket is a Cobb-Douglas composite given by

$$
x_{i, k} \equiv \prod_{j \in I} x_{i j, k}^{g_{i j}}
$$

where $x_{i j, k}$ is the amount it purchases of the sectoral commodity $j, g_{i j} \in[0,1]$ denotes the share of good $j$ in this composite and $\sum_{j \in I} g_{i j}=1, \forall i \in I$. We let $\mathbf{x}_{i, k} \equiv\left(x_{i 1, k}, \ldots, x_{i N, k}\right)^{\prime}$ denote the 
vector of the firm's intermediate good purchases. The profits of the firm $k$ in sector $i$ are given by

$$
\pi_{i, k}=\left(1-\tau_{i}\right) p_{i, k} y_{i, k}-\ell_{i, k}-\sum_{j \in I} p_{j} x_{i j, k}
$$

where $p_{i, k}$ is its own output price, $p_{j}$ is the price at which it purchases input $x_{i j, k}$, and $\tau_{i}$ is a sector-specific revenue tax (or subsidy). We let labor be the numeraire, and thereby normalize the wage to one.

Firms within a sector are monopolistically competitive. To facilitate this, within each sector $i \in I$, we assume there is a producer who aggregates sectoral goods according to the following CES production function with elasticity of substitution $\theta_{i}$ :

$$
y_{i}=\left[\int y_{i, k}^{\frac{\theta_{i}-1}{\theta_{i}}} d k\right]^{\frac{\theta_{i}}{\theta_{i}-1}} .
$$

This aggregator firm is perfectly-competitive, i.e. it takes all prices as given, and maximizes profits given by $\bar{\pi}_{i}=p_{i} y_{i}-\int p_{i, k} y_{i, k} d k$ where $p_{i}$ is the price of the good $i$. We include this producer-which has zero value added and makes zero profits - for exposition only: it ensures that a homogenous good is produced by each industry, while at the same time allowing for monopolistic competition among firms within the industry.

At the economy-wide level, we let $\boldsymbol{\alpha}=\left(\alpha_{1}, \alpha_{2}, \ldots, \alpha_{N}\right)^{\prime}$ denote the vector of sectoral labor shares and $\mathbf{G}$ denote the $N \times N$ input-output matrix with entries $g_{i j}$. The rows of $\mathbf{G}$ sum to one. ${ }^{5}$

The Household. The preferences of the representative household are given by

$$
U(C)-V(L)
$$

where $C$ is its final consumption basket and $L$ its labor supply. We assume the typical regularity conditions: $U$ and $V$ are twice differentiable with $U^{\prime}, V^{\prime}>0, U^{\prime \prime}<0, V^{\prime \prime}>0$, and satisfy the Inada conditions. The final consumption basket is a Cobb-Douglas composite of the sectoral goods:

$$
\mathcal{C}\left(c_{1}, \ldots, c_{N}\right)=\prod_{i \in I} c_{i}^{v_{i}}
$$

The coefficient $v_{i} \in[0,1]$ is the household's expenditure share on good $i$ and $\sum_{j=1}^{N} v_{j}=1$. We denote the vector of household expenditure shares by $\mathbf{v}=\left(v_{1}, v_{2}, \ldots, v_{N}\right)^{\prime}$. The household owns

\footnotetext{
${ }^{5}$ The column sum of $\mathbf{G}$ is its weighted outdegree: the share of sector $i$ 's output in the input uses of the other sectors in the economy.
} 
all firms; its budget constraint is given by

$$
\sum_{i \in I} p_{i} c_{i} \leq L+\sum_{i \in I}\left[\int \pi_{i k} d k+\bar{\pi}_{i}\right]+T .
$$

The left hand side is total expenditure on consumption; the right hand side includes the wage income of the household, dividends from all firms, and lump sum transfers.

Distortions, Rebates, and Market Clearing. There are two types of sectoral distortions in our economy: revenue taxes and monopolistic markups. We use these as simple accounting devices for any type of sectoral distortion. It will however matter for allocations whether the proceeds resulting from these distortions, i.e. tax revenue or profits, are rebated back to the household.

To allow for this possibility we assume that only a fraction $1-\delta_{i}$ of tax proceeds collected from sector $i$ are rebated lump-sum to the household so that $T=\sum_{i \in I}\left(1-\delta_{i}\right) \tau_{i} p_{i} y_{i}$. The remainder $\delta_{i} \in[0,1]$ is thrown out; we let $h_{i} \equiv \delta_{i} \tau_{i} y_{i}$ denote sector $i$ 's output that is wasted.

As a result, the uniform commodity produced by sector $i$ may be either consumed by the household, used as intermediate goods, or thrown out. Goods market clearing is thus given by

$$
y_{i}=c_{i}+h_{i}+\sum_{j \in I} x_{j i}, \quad \forall i \in I
$$

where $x_{j i} \equiv \int x_{j i, k} d k$ is the total amount of good $i$ purchased by all firms in sector $j$. Labor-market clearing satisfies $L=\sum_{i \in I} \ell_{i}$, with $\ell_{i} \equiv \int \ell_{i, k} d k$.

\section{Equilibrium Definition and Characterization}

We will consider the equilibrium effects of shocks to sectoral productivities and distortions. We begin by first defining the aggregate state. Let $\mathbf{z} \equiv\left(z_{1}, \ldots, z_{N}\right)^{\prime}$ denote the vector sectoral productivities, $\boldsymbol{\tau} \equiv\left(\tau_{1}, \ldots, \tau_{N}\right)^{\prime}$ and $\boldsymbol{\theta} \equiv\left(\theta_{1}, \ldots, \theta_{N}\right)^{\prime}$ the vectors of taxes and elasticities of substitution, respectively, and $\boldsymbol{\delta} \equiv\left(\delta_{1}, \ldots, \delta_{N}\right)^{\prime}$ the vector of waste parameters. We assume all of these objects are exogenous and stochastic.

We thus let $s \equiv(\mathbf{z}, \boldsymbol{\tau}, \boldsymbol{\theta}, \boldsymbol{\delta})$ denote the aggregate state. Nature draws $s \in S$ from some arbitrary distribution, $\mu(s) \in \Delta(S)$. We represent an allocation in this economy, $\xi \equiv(\xi(s))_{s \in S}$, by a set of functions,

$$
\xi(s) \equiv\left\{\left(\left(y_{i, k}(s), \ell_{i, k}(s), \mathbf{x}_{i, k}(s)\right)_{k \in[0,1]}, c_{i}(s), h_{i}(s)\right)_{i \in I}, C(s), L(s)\right\}
$$

mapping the realization of the state to quantities chosen by the firms in each sector, the amount 
consumed and thrown out of each good, and aggregate quantities. We similarly represent a price system $\varrho \equiv(\varrho(s))_{s \in S}$ as a set of functions,

$$
\varrho(s) \equiv\{\mathbf{p}(s), P(s), 1\}
$$

mapping the realization of the state to a vector of sectoral prices, $\mathbf{p}(s) \equiv\left(p_{1}(s), \ldots, p_{N}(s)\right)^{\prime}$, the aggregate price level (the price of the household's consumption bundle), and the wage which has been normalized to one. An equilibrium is defined as follows.

Definition 1. An equilibrium is an allocation and a price system, $(\xi, \varrho)$, such that for any realization of $s \in S$,

(i) the firms' output and input bundles maximize firm profits;

(ii) the household's consumption bundle and labor supply are chosen optimally;

(iii) all markets clear.

The set of equilibrium allocations may be characterized as follows.

Proposition 1. An allocation $\xi$ is part of an equilibrium if and only if there exists a set of functions $\phi_{i}, \psi_{i}, q_{i}: S \rightarrow \mathbb{R}_{+}$, for all $i \in I$ defined by:

$$
\begin{gathered}
\phi_{i}(s) \equiv\left(\frac{\theta_{i}(s)-1}{\theta_{i}(s)}\right) \frac{1-\tau_{i}(s)}{1-\delta_{i}(s) \tau_{i}(s)}, \quad \psi_{i}(s) \equiv 1-\delta_{i}(s) \tau_{i}(s), \quad \text { and } \\
q_{i}(s) \equiv \psi_{i}(s) y_{i}(s)=\psi_{i}(s) z_{i}(s) \ell_{i}(s)^{\alpha_{i}} x_{i}(s)^{1-\alpha_{i}}
\end{gathered}
$$

such that the allocation and these functions jointly satisfy the following set of conditions:

$$
\begin{aligned}
& V^{\prime}(L(s))=\phi_{i}(s) U^{\prime}(C(s)) v_{i} \frac{C(s)}{c_{i}(s)} \alpha_{i} \frac{q_{i}(s)}{\ell_{i}(s)}, \quad \forall i \in I, k \in[0,1], s \in S, \\
& \frac{v_{j} C(s) / c_{j}(s)}{v_{i} C(s) / c_{i}(s)}=\phi_{i}(s)\left(1-\alpha_{i}\right) g_{i j} \frac{q_{i}(s)}{x_{i j}(s)}, \quad \forall i, j \in I, k \in[0,1], s \in S,
\end{aligned}
$$

and resource constraints,

$$
L(s)=\sum_{i \in I} \ell_{i}(s) \text { and } q_{i}(s)=c_{i}(s)+\sum_{j \in I} x_{j i}(s), \forall i \in I
$$

Proposition 1 fully characterizes the set of equilibrium allocations by introducing two functions, $\phi_{i}(s)$ and $\psi_{i}(s)$, as transformations of the underlying distortions. The function $\psi_{i}(s)$ defined in (6) represents the fraction of resources thrown out. If we redefine quantities of production from $y_{i}(s)$ to $q_{i}(s)$ via equation $(7)$, then $\psi_{i}(s)$ appears isomorphic to a productivity shock, $z_{i}(s)$. 
This is because the private incentives of a firm are affected in the same way by a distortion as a productivity shock. Moreover, from a social standpoint these resources are lost. This consideration allows one to interpret $q_{i}(s)$ as produced quantities net of wasted resources, while $y_{i}(s)$ represents gross production. Resource constraints may thereby be restated as in (10) with net production equated to total usage of the good as consumption and intermediates. This transformation thereby makes evident the allocational equivalence between $\psi_{i}(s)$ and $z_{i}(s)$.

Next consider the set of equilibrium conditions in (8) and (9). As all firms within a sector are identical, they make identical choices and we henceforth drop the subscript $k$. The first set of equilibrium conditions in (8) indicate that the marginal rate of substitution between consumption of any good and labor is equated to their marginal rate of transformation modulo a wedge denoted by $\phi_{i}(s)$. The second set of equilibrium conditions in (9) similarly indicates that the marginal rate of substitution between any two goods should equal their marginal rate of transformation, again modulo the same wedge. The distortionary wedge $\phi_{i}(s)$ is simply the result of the revenue tax and the monopolistic markup; it is defined in (6) in terms of net quantities rather than gross quantities. ${ }^{6}$

In summary, a simple change-of-variables makes evident the allocational distinction between distortions whose proceeds are rebated to the household and those whose proceeds are not. We henceforth refer to $\phi_{i}(s)$ as the rebated distortion of sector $i$ and to $\psi_{i}(s)$ as its wasted distortion. We let $\boldsymbol{\phi}=\left(\phi_{1}, \ldots, \phi_{N}\right)^{\prime}$ and $\boldsymbol{\psi}=\left(\psi_{1}, \ldots, \psi_{N}\right)^{\prime}$ denote the vectors of realized distortions and in light of this transformation, we henceforth reduce the aggregate state to $s=(\mathbf{z}, \boldsymbol{\phi}, \boldsymbol{\psi})$.

Financial Frictions as Distortions. The reduced-form distortions $\phi$ and $\boldsymbol{\psi}$ have thus far been microfounded as the result of monopolistic-competition and revenue taxes. However, later on in our quantitative application we consider a different source of distortions: financial frictions. To anticipate this interpretation, we consider the following extension of the baseline model; we call this the financial frictions economy.

To simplify, we abstract from both revenue taxes and markup distortions: $\tau_{i}(s)=0$ and $\theta_{i}(s) \rightarrow$ $\infty, \forall i \in I, s \in S$. Firms face a limited enforcement constraint in which they must pay for all working capital costs, i.e. intermediate good purchases and the wage bill, upfront at the beginning of the period. As firm sales are not realized until the end of the period, firms must take out an intraperiod loan in order to finance its working capital. Specifically, firms borrow from a banking sector

\footnotetext{
${ }^{6}$ The left hand side of condition (8) is the marginal cost of labor: the marginal disutility of labor of the household. The right hand side is the marginal benefit of labor times the wedge. The marginal benefit consists of three multiplicative components: the marginal product of labor in the production of commodity $i$, the marginal product of good $i$ in the production of the final good, and the marginal utility of consumption of the final good. Similarly, the left hand side of equation (9) is the ratio of marginal utilities from consumption of either good, simply their marginal rate of substitution. The right hand side is the marginal product of good $i$ in the production of good $j$ times the distortionary wedge. Finally, the primitive wedge is given by $\left(\frac{\theta_{i}(s)-1}{\theta_{i}(s)}\right)\left(1-\tau_{i}(s)\right)$. However, due to the fact that some tax proceeds are thrown out, $\phi_{i}(s)$ defined in (6) recasts the wedge in terms of the net quantities.
} 
at an exogenous sector-specific intra-period interest rate of $1+r_{i}$, implying end-of-period profits $\pi_{i, k}=p_{i, k} y_{i, k}-\left(1+r_{i}\right)\left(\ell_{i}+\sum_{j \in I} p_{j} x_{i j, k}\right)$. We furthermore assume that all interest payments accrued to the banking sector are rebated lump-sum to the household in the form of bank profits. Equilibrium allocations in this economy may be characterized as follows. ${ }^{7}$

Proposition 2. In the financial frictions economy, an allocation $\xi$ is part of an equilibrium if and only if the conditions in Proposition 1 hold, with $\boldsymbol{\phi}$ and $\boldsymbol{\psi}$ redefined as follows:

$$
\phi_{i}(s) \equiv \frac{1}{1+r_{i}(s)}, \quad \text { and } \quad \psi_{i}(s) \equiv 1, \quad \forall i \in I, s \in S
$$

Therefore, rebated distortions may likewise emerge from working capital constraints. Note that financial friction severity is related to the intra-period cost of borrowing: if a sector's cost of borrowing is high, firms in that sector are more distorted. Furthermore, if a portion of bank profits were thrown out rather than paid to the household, then this portion would manifest as wasted distortions.

A clear limitation of our simplified model of financial frictions is that we abstract completely from how firms obtain credit. Specifically, working capital constraints are entirely exogenous, and we allow only for bank credit but no trade credit. We refer the reader to Altinoglu (2018), Reischer (2019), and Luo (2016) for models of endogenous financial constraints and supplier trade credit.

\section{Aggregation}

We now demonstrate that there exists an isomorphism between the multi-sector production network economy described above and a certain representative firm, representative household economy. For any vector $\mathbf{w} \in \mathbb{R}_{+}^{N}$, let us define $\operatorname{diag}(\mathbf{w})$ as a square matrix with the elements of vector $\mathbf{w}$ on the main diagonal and all off-diagonal elements equal to zero. We obtain the following aggregation.

Theorem 1. There exists functions TFP:S $\rightarrow \mathbb{R}_{+}$and $\Lambda: \mathbb{R}_{+}^{N} \rightarrow \mathbb{R}_{+}$such that equilibrium quantities aggregate to a prototypical representative firm, representative household economy with a constant returns-to-scale aggregate production function given by

$$
C(s)=T F P(s) L(s),
$$

\footnotetext{
${ }^{7}$ There exist multiple ways to model financial frictions, most notably collateral constraints as in Bernanke and Gertler (1989) and Kiyotaki and Moore (1997) and frictions in the financial intermediation sector as in Gertler and Karadi (2011). We abstract from these types of constraints; instead our model is closer in spirit to the intraperiod working capital constraints present in Jermann and Quadrini (2011) and Bigio (2015) but with the addition of intermediate good purchases.
} 
and an equilibrium intratemporal condition given by

$$
\frac{V^{\prime}(L(s))}{U^{\prime}(C(s))}=\Lambda(\phi(s)) \frac{C(s)}{L(s)} .
$$

(i) The aggregate labor wedge is given by

$$
\Lambda(\boldsymbol{\phi}(s)) \equiv \boldsymbol{\alpha}^{\prime}\left\{\operatorname{diag}(\boldsymbol{\phi}(s))^{-1}-\mathbf{G}^{\prime} \operatorname{diag}(\mathbf{1}-\boldsymbol{\alpha})\right\}^{-1} \boldsymbol{v}
$$

and satisfies $\Lambda(\mathbf{1})=1$.

(ii) Total factor productivity is given by

$$
T F P(s) \equiv \kappa A(\mathbf{z}(s)) A(\boldsymbol{\psi}(s)) A(\phi(s)) \Lambda(\phi(s))^{-1},
$$

where $\kappa>0$ is a strictly-positive scalar and for any vector $\mathbf{w} \in \mathbb{R}_{+}^{N}$, the function $A: \mathbb{R}_{+}^{N} \rightarrow \mathbb{R}_{+}$is a log-linear operator defined by

$$
\log A(\mathbf{w}) \equiv \boldsymbol{a}^{\prime} \log \mathbf{w}
$$

where $\boldsymbol{a}^{\prime} \equiv \mathbf{v}^{\prime} \mathbf{B}$ and $\mathbf{B}$ is the Leontief inverse matrix:

$$
\mathbf{B} \equiv\left[\mathbb{I}_{N}-\operatorname{diag}(\mathbf{1}-\boldsymbol{\alpha}) \mathbf{G}\right]^{-1}
$$

Furthermore, this function satisfies $A(\mathbf{1})=1$.

Theorem 1 establishes that for the class of economies we consider-static, multi-sector, CobbDouglas input-output economies with sectoral wasted and rebated distortions - equilibrium allocations aggregate to a prototypical representative-firm, representative-household economy. An equilibrium allocation satisfies a CRS aggregate technology and an intratemporal condition. Using the the terminology of Chari et al. (2007), Theorem 1 establishes that the sectoral shocks manifest as either movements in the aggregate efficiency wedge, the aggregate labor wedge, or both. The efficiency wedge, also known as total factor productivity (TFP), is the multiplicative term in the CRS aggregate production function in (12). The labor wedge in (13) distorts the household's marginal rate of substitution between consumption and labor away from the economy's marginal rate of transformation. ${ }^{8}$

How much each sectoral shock matters in the aggregate depends on both the type of shock and the underlying input-output network. Notably, TFP is a log-separable function of all three types

\footnotetext{
${ }^{8}$ The left hand side of (13) is simply the representative household's marginal rate of substitution between consumption and labor. The right-hand side consists of two components: (i) the economy's marginal rate of transformation between consumption and labor - i. e. the representative firm's marginal product of labor - and the aggregate labor wedge.
} 
of underlying shocks, $\mathbf{z}, \boldsymbol{\psi}, \boldsymbol{\phi}$, while the labor wedge is a function of the rebated distortions, $\phi$, alone. The log-separability of these components is a by-product of the Cobb-Douglas assumption on preferences and technologies. ${ }^{9}$

Theorem 1 thereby generalizes the aggregation results of Jones (2013) to allow for both wasted distortions and an elastic labor supply. ${ }^{10}$ If the primary factor in our model were inelastic, as it is in Jones (2013), then there would be no labor wedge and sectoral rebated distortions would manifest in TFP alone. The aggregation of the rebated distortions into the labor wedge is therefore a novel contribution of this paper; we show in due course that this generalization is of first-order importance in terms of the macroeconomic effects of distortions.

In what follows we consider the three types of sectoral shocks and how each may lead to aggregate movements in the economy.

\subsection{The aggregate effects of productivity shocks}

We first consider the aggregate effects of sectoral productivity shocks. Productivities affect the aggregate allocation through TFP alone; their total effect is given by the term $A(\mathbf{z}(s)$ ), written in logs here:

$$
\log A(\mathbf{z}(s))=\boldsymbol{a}^{\prime} \log \mathbf{z}(s)=\mathbf{v}^{\prime} \mathbf{B} \log \mathbf{z}(s)
$$

where $\mathbf{B}$ denotes the Leontief inverse matrix (Burress, 1994). As $A(\cdot)$ is a log-linear operator, the total effect of each shock on aggregate TFP is succinctly represented by the vector of constants $\boldsymbol{a}$; each component gives the cumulative network effect of an individual productivity shock:

$$
\frac{d \log A(\mathbf{z}(s))}{d \log z_{i}}=a_{i}=\mathbf{v}^{\prime}\left[\mathbb{I}_{N}-\operatorname{diag}(\mathbf{1}-\boldsymbol{\alpha}) \mathbf{G}\right]^{-1} e_{i}
$$

where $e_{i}$ is a column vector of length $N$ composed of all zeros except a 1 as its $i$ th element. ${ }^{11}$ Acemoglu et al. (2012) terms this vector the "influence vector." 12 However, in order to delineate across the three different types of shocks and the two distinct aggregate channels in our paper, we more specifically refer to it as the "TFP influence of productivities," or the "TFP influence vector" for short. Note that the TFP influence vector is independent of the aggregate state; it is a function

\footnotetext{
${ }^{9}$ By log-separability of TFP, we simply mean that in logs it is additively-separable in the three types of shocks:

$$
\log T F P(s)=\log A(\mathbf{z}(s))+\log A(\boldsymbol{\psi}(s))+\log \Phi(\phi(s))
$$

where $\log \Phi(\phi(s)) \equiv \log A(\phi(s))-\log \Lambda(\phi(s))$. This property is non-generic, it is the result of the Cobb-Douglas assumption; we refer the reader to Baqaee and Farhi (2019a) for results with more general production functions.

${ }^{10}$ We may nest the closed-economy version of Jones (2013) by assuming in our model that labor supply is fixed and by turning off the $\psi$ shocks. Formally we may do this by assuming infinitely inelastic labor supply and setting $\delta=0$.

${ }^{11}$ Here the column vector $e_{i}$ simply chooses the $i$ th element of the vector $\boldsymbol{v}^{\prime} \mathbf{B}$.

${ }^{12}$ We may nest the economy in Acemoglu et al. (2012) as a special case of our model as follows. We assume that the labor share is constant across all sectors and that the household's consumption shares are constant and equal across all commodities: $\alpha_{i}=\bar{\alpha}$ and $v_{i}=1 / N$, for all $i$. In this case, the TFP influence vector reduces $\frac{1}{N} \mathbf{1}^{\prime}\left[\mathbb{I}_{N}-(1-\bar{\alpha}) \mathbf{G}\right]^{-1}$ as in Acemoglu et al. (2012).
} 
of the network structure and the primitives alone.

To gain intuition for the TFP influence vector, one may expand the Leontief inverse matrix:

$$
\left[\mathbb{I}_{N}-\operatorname{diag}(\mathbf{1}-\boldsymbol{\alpha}) \mathbf{G}\right]^{-1}=\mathbb{I}_{N}+\operatorname{diag}(\mathbf{1}-\boldsymbol{\alpha}) \mathbf{G}+(\operatorname{diag}(\mathbf{1}-\boldsymbol{\alpha}) \mathbf{G})^{2}+\cdots
$$

Consider a negative productivity shock to sector $i$. The first term in this expansion yields the direct effect on this sector: in response to a negative productivity shock, sectoral output falls and simultaneously its price rises. This price increase implies that the downstream customers of sector $i$, i.e. the sectors that purchase sector $i$ 's good and use it as an input, buy less of that input in equilibrium and thereby produce less output. The second term in the above expansion thus gives the secondary effect of the shock on sector $i$ 's customers: their output falls and simultaneously their price increases. But this implies that those sectors' downstream customers also buy less inputs, produce less output, and increase their own output prices. The effect on sector $i$ 's customers' customers is thereby given in the third term of this expansion, and so on. Therefore, the Leontief Inverse matrix captures the entire infinite hierarchy of network effects; the extent to which a productivity shock propagates through the network and manifests as aggregate movement in TFP depends crucially on the input-output structure embedded in G.

The Leontief inverse matrix is closely related to the mathematical notion of Bonacich centrality. Thus, productivity shocks in sectors with high centrality — sectors with more direct and/or indirect downstream customers - have larger effects on TFP than shocks to sectors with low centrality. We refer the reader to Acemoglu et al. (2012) and Carvalho (2014) for more discussion on this topic. ${ }^{13}$

\subsection{The aggregate effects of wasted distortions}

We next consider the aggregate effects of wasted distortions, $\boldsymbol{\psi}(s)$. As previously established in Proposition 1, these shocks are isomorphic to productivity shocks. As such, the sole channel through which these shocks operate is via TFP; their total effect on TFP is likewise given by the term $A(\boldsymbol{\psi}(s))$. Given that neither the wasted distortions nor productivity shocks contribute to the aggregate labor wedge, the following corollary of Theorem 1 is immediate.

Corollary 1. Consider the limit of our economy in which there are no markups and all tax proceeds are wasted: $\phi_{i}(s) \rightarrow 1$ and $\psi_{i}(s) \rightarrow 1-\tau_{i}(s), \forall i \in I$. In this limit, the prototypical representative firm, representative household economy operates an aggregate production function $C(s)=A(\mathbf{z}(s)) A(\boldsymbol{\psi}(s)) L(s)$, and there is no labor wedge: $\Lambda(\mathbf{1})=1$.

\footnotetext{
${ }^{13}$ To be clear, the aggregate effects of productivity shocks in a network economy are not a novel part of our paper; they have been studied extensively in the literature. The results in (18) merely reproduce results previously derived in Burress (1994); Gabaix (2011); Acemoglu et al. (2012).
} 


\subsection{The aggregate effects of rebated distortions}

We now consider the aggregate effects of the rebated distortions, $\phi(s)$. Theorem 1 establishes that rebated distortions manifest as movements in both TFP and the labor wedge.

Rebated distortions and the labor wedge. The economics behind the emergence of the aggregate labor wedge is rather intuitive. The household itself faces no distortion: its marginal rate of substitution between consumption and labor is equated with the real wage. On the other hand, sectoral distortions drive a wedge between each producer's marginal revenue and marginal cost. Such a wedge causes firms within a sector to purchase less inputs, produce less output, and charge a higher price. Downstream sectors thereby face higher input prices and hence higher marginal costs. If these sectors are in turn subject to their own distortion, this causes them to further reduce output and raise prices above their already distorted marginal costs. This raises input costs for their downstream customers, and so on. As a result, the price of the final consumption good is distorted above its marginal cost of production. Equivalently, the real wage is lower than the marginal product of labor; this implicit wedge constitutes the labor wedge. ${ }^{14}$

Unlike the $A(\cdot)$ function discussed above, the aggregate labor wedge function $\Lambda(\cdot)$ is not $\log$ linear. As a consequence, the aggregate effect of a sectoral distortion cannot be summarized by a constant as in the case of productivity shocks. To see this and to mirror the terminology introduced above for productivity shocks, we define the "labor wedge influence of distortions" as

$$
\chi_{i}(s) \equiv \frac{d \log \Lambda(\phi(s))}{d \log \phi_{i}}
$$

and let $\chi(s)=\left(\chi_{1}(s), \ldots, \chi_{N}(s)\right)^{\prime}$ denote the realized vector of these influences. Unlike $\boldsymbol{a}$, this vector depends jointly on the input-output network structure, $\mathbf{G}$, as well as the realized vector of distortions $\phi(s)$. We explore what factors contribute to labor wedge influence at the end of this section and later on with a few simple examples. Prior to that, we consider TFP.

Rebated distortions and TFP. As noted above, TFP is log-separable in the three types of underlying shocks. Thus, let the function $\Phi: \mathbb{R}_{+}^{N} \rightarrow \mathbb{R}_{+}$denote the component of TFP that is due to sectoral rebated distortions alone: $\Phi(\phi(s)) \equiv A(\phi(s)) \Lambda(\phi(s))^{-1}$. We may thus rewrite TFP as follows:

$$
\begin{gathered}
\log \operatorname{TFP}(s)=\log A(\mathbf{z}(s))+\log A(\boldsymbol{\psi}(s))+\log \Phi(\phi(s)), \\
\text { where } \quad \log \Phi(\phi(s)) \equiv \log A(\phi(s))-\log \Lambda(\phi(s)) .
\end{gathered}
$$

\footnotetext{
${ }^{14}$ A large literature has documented significant movement of the labor wedge at business cycle frequency (see e.g. Hall, 1997; Rotemberg and Woodford, 1999; Chari et al., 2007).
} 
Consider the first term on the right hand side of (23), that is, $\log A(\phi(s))$. This term is identical to the term that captures the aggregate TFP effects of productivity shocks and wasted distortions. This is because all three types of shocks - productivity shocks, wasted distortions, and rebated distortions - affect individual firms' optimality conditions in the same way; it is the aggregation of these optimality conditions that produces these identical components. ${ }^{15}$

To understand this, recall that the way in which sectoral productivity shocks propagate through the system is through movements in prices - movements which translate to movements in input costs for downstream customers, downstream customers' customers, and so on. But note that shocks to distortions lead to movements in prices in much the same way. The downstream effects of these price movements remain the same regardless of whether the original culprit is a rebated distortion, a wasted distortion, or a productivity shock; in fact, the vector of equilibrium prices is given by

$$
\log \mathbf{p}(s)=-\mathbf{B}[\log \mathbf{z}(s)+\log \boldsymbol{\psi}(s)+\log \boldsymbol{\phi}(\mathbf{s})]
$$

where $\mathbf{B}$ is the Leontief inverse matrix. Thus, all three types of shocks are equivalent in terms of their equilibrium price effects.

However, despite inducing equivalent movement in equilibrium prices, rebated distortions are unlike productivity shocks and wasted distortions in that they do not actually destroy resources: all production possibilities remain intact. ${ }^{16}$ It is for this reason that the second term in $(23), \log \Lambda(s)$, emerges. Although distortions alter a sector's input and output decisions, the excess revenue created by this behavior is transferred lump-sum to the household; it is this increase in household spending that accounts for the second term. The second term thereby acts as a countervailing force against the first: household demand dampens the TFP effect of rebated distortions on relative to that of productivity shocks or wasted distortions. ${ }^{17}$

Taken together, the only way by which rebated distortions generate a fall in TFP is through input misallocation: rebated distortions lower the productive efficiency of the economy by altering the flow of inputs away from their most efficient uses. We illustrate this point next.

\footnotetext{
${ }^{15}$ To see this directly, consider the equilibrium conditions in (8) and (9). It is clear that a fall in $\phi_{i}(s)$ in these conditions is isomorphic to a fall in $q_{i}(s)$ due to either $z_{i}(s)$ or $\psi_{i}(s)$.

${ }^{16}$ To see this directly, recall the resource constraint in (10): while a fall in either $z_{i}(s)$ or $\psi_{i}(s)$ has a direct effect on this constraint, the $\phi_{i}(s)$ distortion is entirely absent.

${ }^{17}$ This point highlights the crucial difference between the rebated distortions and the wasted distortions. While the $\boldsymbol{\psi}(s)$ distortions affect individual firm decisions in the same way as $\phi(s)$ distortions, the excess revenue of the former are discarded while those of the latter are rebated. As a result, there is no increase in household spending from $\boldsymbol{\psi}(s)$ to offset the firms' incentives.
} 


\subsection{The first-order effects of rebated distortions}

We now examine the first-order effects of rebated distortions. We show that for small perturbations, rebated distortions have zero first-order effects on TFP but non-zero first-order effects on the labor wedge.

Again invoking its log-separability, we need only consider the component of TFP that is due to rebated distortions: $\Phi(\phi(s))$. A first-order log approximation of this component around any vector $\phi(s)$ is given by:

$$
d \log \Phi(\phi(s))=\left\{\left.\frac{d \log A(\phi(s))}{d \log \phi_{i}}\right|_{\phi(s)}-\left.\frac{d \log \Lambda(\phi(s))}{d \log \phi_{i}}\right|_{\phi(s)}\right\} d \log \phi_{i} .
$$

We say that an equilibrium allocation is efficient if $\boldsymbol{\phi}=\mathbf{1} .{ }^{18}$ Evaluating the above expression at $\phi=1$ yields the following result.

Theorem 2. To a first-order log approximation around efficiency, $\boldsymbol{\phi}=\mathbf{1}$ :

(i) Rebated distortions have zero first-order effects on TFP.

$$
\left.\frac{d \log \Phi(\phi(s))}{d \log \phi_{i}}\right|_{\phi=1}=0, \quad \forall i \in I
$$

(ii) Rebated distortions have non-zero first-order effects on the labor wedge. Moreover, the first-order effects of rebated distortions on the labor wedge are equal to the TFP influence of productivities.

$$
\left.\frac{d \log \Lambda(\phi(s))}{d \log \phi_{i}}\right|_{\phi=\mathbf{1}}=\left.\frac{d \log A(\phi(s))}{d \log \phi_{i}}\right|_{\phi=\mathbf{1}}=a_{i}, \quad \forall i \in I .
$$

Part (i) of Theorem 2 should come as no surprise: this result holds in more general economies and is simply a consequence of the envelope theorem, see Baqaee and Farhi (2019a). ${ }^{19}$ The equilibrium allocation at $\phi=\mathbf{1}$ maximizes productive efficiency in the Diamond and Mirrlees (1971) sense. By the envelope theorem, small distortions generate zero first-order effects in productive efficiency; misallocation manifests in second and higher-order terms alone. ${ }^{20}$

This result highlights another stark difference between rebated distortions, wasted distortions, and productivity shocks. While rebated distortions have only second and higher-order effects on

\footnotetext{
${ }^{18}$ It is clear from the first welfare theorem that the equilibrium allocation with $\phi=\mathbf{1}$ coincides with the welfaremaximizing choice of the planner. We need not take a stand on the vector of wasted distortions. It is a matter of interpretation whether the wasted distortions are controlled by the planner; however, due to the log-separability of TFP, the vector of wasted distortions does not matter for our subsequent results.

${ }^{19}$ A corollary of Baqaee and Farhi (2019a)'s main theorem pertains to the first-order effects of productivities and distortions on TFP at the efficient benchmark. Our results in part (i) of Theorem 2 coincide with theirs albeit in the more restricted Cobb-Douglas economy. Baqaee and Farhi (2019a) further characterize the first-order effects of distortions and productivity shocks on TFP away from efficiency.

${ }^{20}$ Jones (2013) writes, "small departures from the optimal allocation of labor have tiny effects on TFP (an application of the envelope theorem), but significant misallocation can have very large effects."
} 
TFP, the latter two types of shocks result in first-order TFP movement. The first-order TFP effects of wasted distortions and productivity shocks are captured by the elements of the TFP influence vector given in (19); this is true not only at the efficient allocation but globally.

Consider now part (ii) of Theorem 2. Recall that in general the labor wedge influence of distortions, defined in (21), depends jointly on the network structure as well as the entire realized distribution of rebated distortions. If we now define $\left.\chi_{i}^{*} \equiv \chi_{i}(s)\right|_{\phi=1}$ as the "labor wedge influence of distortions at efficiency" and we let $\chi^{*}$ denote the vector of such influences, part (ii) of Theorem 2 tells us that to a first order approximation,

$$
\chi_{i}^{*}=a_{i}=\mathbf{v}^{\prime}\left[\mathbb{I}_{N}-\operatorname{diag}(\mathbf{1}-\boldsymbol{\alpha}) \mathbf{G}\right]^{-1} e_{i}, \quad \forall i \in I
$$

That is, the labor wedge influence of distortions at efficiency is approximately equal to the TFP influence of productivities! This implies that the same intuition for how sectoral productivity shocks affect TFP applies equally well to how small rebated distortions affect the aggregate labor wedge. Small distortions in sectors with high centrality - sectors with more direct and/or indirect downstream customers - have larger effects on the aggregate labor wedge than small distortions in sectors with low centrality. Yet, despite heterogeneity in their aggregate labor wedge influence, all rebated distortions have zero first-order effects on TFP.

There are two quantitative implications of this result. The first concerns how one may measure the labor wedge influence of distortions. Applying Hulten (1978)'s theorem for productivities to our result on distortions, we immediately obtain the following result.

Corollary 2. At the efficient benchmark, a sufficient statistic for the first-order effects of sectoral rebated distortions on the aggregate labor wedge are the Domar weights:

$$
\chi_{i}^{*}=\lambda_{i} \quad \text { where } \quad \lambda_{i} \equiv \frac{p_{i} y_{i}}{C} \quad \forall i \in I
$$

Domar weights are sectoral sales as a share of GDP; in any economy with intermediate good trade, the sum of these weights across sectors is greater than 1. In an efficient economy, the Domar weights are sufficient statistics for the first-order effects of sectoral productivities on TFP (Hulten, 1978). Given that the first-order effects of productivities on TFP are equal to the first-order effects of distortions on the labor wedge near efficiency, and given that the Domar weights are sufficient statistics for the former, we may also infer that they are sufficient statistics for the latter.

The second quantitative implication is the following. Consider an advanced economy with little misallocation. In this economy, relative to TFP the labor wedge may in fact be the quantitatively stronger aggregate channel by which sectoral distortions manifest. We return to this possibility in Section 6 . 


\section{$5 \quad$ Simple Network Economies}

While the results for small distortions may be helpful for intuition, they hold only up to a first-order approximation around efficiency. To the extent that the economy is away from efficiency, network centrality is no more sufficient to capture these effects. Instead, the entire topology of the network matters in conjunction with the distribution of shocks.

We explore some possibilities in this section. We warm-up with two extremely simple network structures: the first is an economy in which production is organized via a vertical supply chain; the second is a horizontal economy with zero intermediate good trade. The vertical and horizontal economies are similar to the "snake" and "spider" economies studied in the Global Value Chains literature (Antràs et al., 2017; Antràs and de Gortari, 2017; Baldwin and Venables, 2013). We then consider a hybrid economy which nests both the vertical and horizontal economies as limit cases. By varying two parameters in this economy, we may move continuously between the vertical and examine some special intermediate cases.

We use these examples to provide intuition on how different network structures result in different ways by which sectoral distortions affect TFP and the labor wedge. The general lesson is that sectors can differ substantially in their influence across the two aggregate channels: in particular, distortions in certain key "bottleneck" sectors can have disproportionately large effects on the aggregate labor wedge but at the same time contribute the least to movements in TFP.

\subsection{The Pure Vertical and the Pure Horizontal Economy}

Throughout this section we abstract from both productivity shocks and wasted distortions, allowing us to focus exclusively on the effects of rebated distortions. ${ }^{21}$ We first consider two economies, each of which consists only of three sectors indexed by $i=1,2,3$.

The pure vertical economy. Sectors are arranged in a vertical supply chain: the first sector uses labor as its sole input to production, while each consecutive sector $i$ uses the intermediate good produced by sector $i-1$ as its sole input. At the end of the supply chain, the third sector produces a final good consumed by the household. Formally, the sectors' production functions are:

$$
y_{1}=\ell, \quad y_{2}=x_{21}, \quad y_{3}=x_{32} .
$$

Market clearing satisfies $x_{21}=y_{1}, x_{32}=y_{2}$, and $C=y_{3} \cdot{ }^{22}$

\footnotetext{
${ }^{21}$ Formally we set the vectors $\mathbf{z}(s)=\psi(s)=(1, \ldots, 1)^{\prime}, \forall s \in S$.

${ }^{22}$ This model is similar to Kalemli-Ozcan et al. (2014) who study financial frictions in vertical economies.
} 
Proposition 3. In the pure vertical economy, TFP and the labor wedge satisfy:

$$
T F P(s)=1 \quad \text { and } \quad \Lambda(\phi(s))=\phi_{1}(s) \phi_{2}(s) \phi_{3}(s) .
$$

In this economy rebated distortions do not induce an aggregate efficiency wedge: there is no movement in TFP not only near the efficient allocation but globally. This is because the pure vertical production structure allows for only one route through which the primary factor, labor, may travel in order to be transformed into the final consumption good. Even if sectoral input choices are distorted, inputs cannot be misallocated away from its one path. As a result, distortions have zero effect on TFP.

While sectoral distortions do not generate any loss in productive efficiency, they do induce a labor wedge. As labor and inputs move downstream along the vertical supply chain en route to becoming the final good, output prices are distorted along the path away from marginal costs. As a result, the price of the final consumption good is distorted above its true marginal cost of production; by implication, a labor wedge - equal to the product of the sectoral distortions - arises. $^{23}$

The pure horizontal economy. Consider now the polar opposite of the previous example: a pure horizontal economy in which there is no intermediate good trade. Each sector hires labor as its sole input and produces a final good consumed by the household. Production functions are:

$$
y_{1}=\ell_{1}, \quad y_{2}=\ell_{2}, \quad y_{3}=\ell_{3}
$$

The goods of each sector are consolidated into a final consumption basket, given by $C=c_{1}^{v_{1}} c_{2}^{v_{2}} c_{3}^{v_{3}}$, where we normalize $v_{3}=1-v_{2}-v_{2}$.

Proposition 4. In the pure horizontal economy, TFP and the labor wedge satisfy:

$$
\begin{gathered}
\operatorname{TFP}(s)=\frac{\phi_{1}(s)^{v_{1}} \phi_{2}(s)^{v_{2}} \phi_{3}(s)^{v_{3}}}{v_{1} \phi_{1}(s)+v_{2} \phi_{2}(s)+v_{3} \phi_{2}(s)}, \quad \text { and } \\
\Lambda(\phi(s))=v_{1} \phi_{1}(s)+v_{2} \phi_{2}(s)+v_{3} \phi_{2}(s)
\end{gathered}
$$

Rebated distortions manifest in both TFP and the labor wedge. Because there are no inputoutput linkages, the extent to which distortions affect TFP and the labor wedge depend only on relative consumption shares.

In this economy an aggregate efficiency wedge arises if and only if rebated distortions are asymmetric. To see this, consider the special case in which the rebated distortions are identical across sectors; suppose there is a non-empty subset of states $\bar{S} \subset S$ defined as follows: $\bar{S} \equiv$

\footnotetext{
${ }^{23}$ Equivalently, the real wage falls relative to the marginal product of labor.
} 


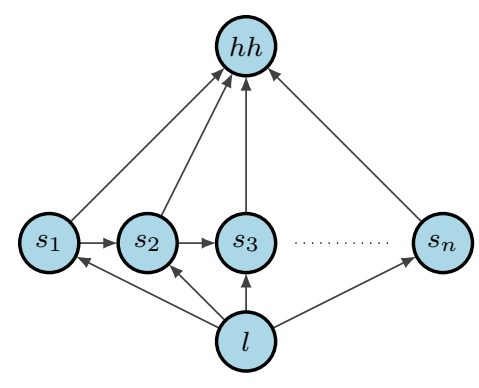

Hybrid Supply Chain

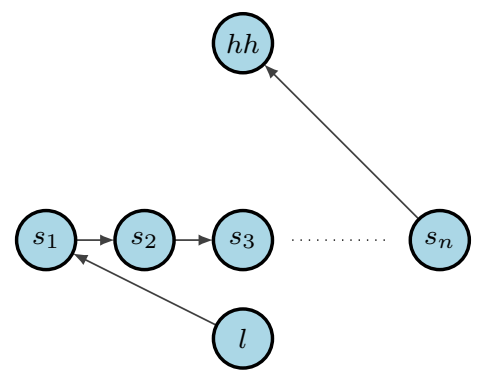

Vertical limit: $\alpha \rightarrow 0, \rho \rightarrow 0$

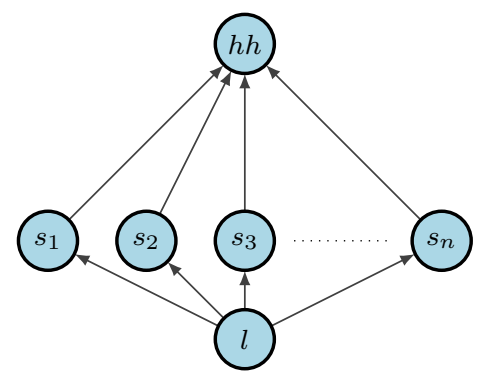

Horizontal limit: $\alpha \rightarrow 1$

Figure 1: The Hybrid Supply Chain Economy and its limits

$\left\{s \in S \mid \phi_{i}(s)=\bar{\phi}(s), \quad \forall i \in I\right\}$. In states in which distortions are symmetric, the economy admits no efficiency wedge and only an aggregate labor wedge:

$$
\operatorname{TFP}(s)=1 \text { and } \Lambda(\phi(s))=\bar{\phi}(s) \quad \text { for all } s \in \bar{S}
$$

If sectors are equally distorted, then labor cannot be misallocated. As a result, there is no loss in productive efficiency à la Diamond and Mirrlees (1971).

In the generic case of asymmetric distortions, an efficiency wedge does emerge. Asymmetries induce labor to move from more distorted sectors to less distorted ones, thereby lowering productive efficiency. The horizontal structure is the structure most typically found in standard business cycle models, and the misallocation of factors due to (rebated) distortions in horizontal CES economies has been studied quite extensively, e.g. Restuccia and Rogerson (2008) and Hsieh and Klenow (2009).

\subsection{The Hybrid Supply Chain Economy}

In the vertical economy, rebated distortions result in an aggregate labor wedge but not an efficiency wedge. This is because there is only one path through which the primary factor may be transformed into consumption. In any generic network, however, there are multiple paths through which primary factors are transformed into final goods. We now demonstrate that the idea that disruptions along a large portion of these paths may have a disproportionately strong effect on the labor wedge but a disproportionately weak effect on the TFP can be extended more generally.

We consider the following, more general, hybrid supply chain economy. There are $N$ sectors with production functions given by

$$
y_{i}=\ell_{i}^{\alpha_{i}} x_{i, i-1}^{1-\alpha_{i}} \text {, where } \alpha_{i}=\alpha^{i-1}, \forall i \in I
$$


for some scalar $\alpha \in(0,1]$. Similar to the vertical economy, each sector uses the intermediate good produced by its neighboring upstream sector as an input to production. Unlike the pure vertical economy, however, each sector produces using labor. The most upstream sector, sector 1 , has the greatest sectoral labor share equal to one and it decreases exponentially at a rate $\alpha$ as one moves down the supply chain.

Similar to the horizontal economy each sector produces a final good sold to the household. The household's expenditure shares are given by

$$
v_{i}=\frac{1}{\xi} \rho^{N-i}
$$

for some scalar $\rho \in(0,1]$, where we set $\xi=\sum_{j=1}^{N} \rho^{N-j}$ in order to normalize the sum to one. Therefore the most downstream sector, sector $N$, has the greatest consumption share equal to $1 / \xi$. The consumption share of each sector decreases exponentially at a rate $\rho$ as one moves up the supply chain. Finally, market clearing is given by $y_{i}=c_{i}+x_{i+1, i}$, for all $i \in\{1, \ldots N-1\}$, and $y_{N}=c_{N}$ for sector $N$. This network is presented in the first panel of Figure 1.

Proposition 5. In the hybrid chain economy, the labor wedge is given by

$$
\Lambda(\phi(s))=\frac{1}{\xi} \sum_{i=1}^{N} \alpha^{i-1}\left(\rho^{N-i} \phi_{i}(s)+\sum_{j=i+1}^{N} \rho^{N-j}\left[\prod_{m=i}^{j-1}\left(1-\alpha^{m}\right) \prod_{m=i}^{j} \phi_{m}(s)\right]\right)
$$

and total factor productivity by $\operatorname{TFP}(s)=A(\phi(s)) \Lambda(\phi(s))^{-1}$ with coefficients

$$
a_{i}=\frac{1}{\xi}\left(\rho^{N-i}+\sum_{j=i+1}^{N} \rho^{N-j}\left[\prod_{m=i}^{j-1}\left(1-\alpha^{m}\right)\right]\right)
$$

The hybrid chain economy is thereby parameterized by only two scalars, $\alpha$ and $\rho$. Proposition 5 characterizes the equilibrium of this economy for any pair of values $(\alpha, \rho) \in(0,1]^{2}$. By varying these two parameters, one may jointly control the degree of "verticality" and "horizontality" in this economy. To see this, we next demonstrate how one may nest both the pure vertical and the pure horizontal economies as particular limit cases of the hybrid economy.

Lemma 1. Consider the following two limits of the hybrid chain economy.

(i) The hybrid chain economy nests the pure vertical economy in the limit in which $\alpha \rightarrow 0$ and $\rho \rightarrow 0$. In this limit, for any vector of rebated distortions, TFP $(s)=1$. Regardless of the state $s$, sectors have equal labor wedge influence: $\chi_{i}(s)=1, \forall i \in I, s \in S$.

(ii) The hybrid chain economy nests the pure horizontal economy when $\alpha=1$ for any $\rho$. In this limit, the labor wedge influence satisfies $\chi_{i}(s) \propto v_{i} \phi_{i}(s), \forall i \in I, s \in S$. 
Consider part (i) of Lemma 1. In this limit, labor is used only by sector 1 and the household consumes only the good produced by sector $N$. This nests the pure vertical economy: there is only one route through which labor is ultimately transformed into the final good, and hence inputs cannot be misallocated. In part (ii) of Lemma 1, the labor share of each sector approaches 1; equivalently, the intermediate good share of each sector approaches zero. This economy thereby nests the pure horizontal economy in which there is no intermediate good trade. These economies are presented in the second and third panels of Figure 1.

Bottlenecks. One can thereby move from the horizontal economy to the vertical economy by reducing both $\alpha$ and $\rho$ to zero. We now consider two intermediate limit cases in which we reduce only one parameter.

Proposition 6. Consider the following two limits of the hybrid chain economy.

(i) The supply-bottleneck. Let $\alpha \rightarrow 0$ for fixed $\rho$. The labor wedge approaches

$$
\Lambda(s)=\frac{1}{\xi}\left(\sum_{i=1}^{N} \rho^{N-i}\left[\prod_{m=1}^{i} \phi_{m}(s)\right]\right)
$$

and the labor wedge influence of distortions satisfies the following strict ordering:

$$
1=\chi_{1}(s)>\chi_{2}(s)>\cdots>\chi_{N}(s), \quad \forall s \in S .
$$

$\operatorname{TFP}(s)$ is a function of $\left\{\phi_{2}(s), \ldots, \phi_{N}(s)\right\}$ but is independent of $\phi_{1}(s), \forall s \in S$.

(ii) The demand-bottleneck. Let $\rho \rightarrow 0$ for fixed $\alpha$. The aggregate labor wedge approaches

$$
\Lambda(s)=\alpha^{N-1} \phi_{N}(s)+\phi_{N}(s) \sum_{i=1}^{N-1} \alpha^{i-1}\left(\prod_{m=i}^{N-1}\left(1-\alpha^{m}\right) \phi_{m}(s)\right)
$$

and the labor wedge influence of distortions satisfies the following strict ordering:

$$
\chi_{1}(s)<\chi_{2}(s)<\cdots<\chi_{N}(s)=1, \quad \forall s \in S .
$$

$\operatorname{TFP}(s)$ is a function of $\left\{\phi_{1}(s), \ldots, \phi_{N-1}(s)\right\}$ but is independent of $\phi_{N}(s), \forall s \in S$.

Consider the limit in part (i) of Proposition 6. In this limit, labor is used only by the most upstream sector: sector $1 .{ }^{24}$ However, the household continues to consume all sectoral goods and as such there remain multiple paths by which labor is transformed into consumption. In contrast, consider the limit featured in part (ii). In this limit, the household consumes only the good produced

\footnotetext{
${ }^{24}$ Note that as $\alpha \rightarrow 0$ the vector of labor shares approaches $\boldsymbol{\alpha} \rightarrow(1,0,0, \cdots, 0)^{\prime}$. Similarly, as $\rho \rightarrow 0$, the vector of expenditure shares approaches $\mathbf{v} \rightarrow(0,0, \cdots 0,1)^{\prime}$.
} 


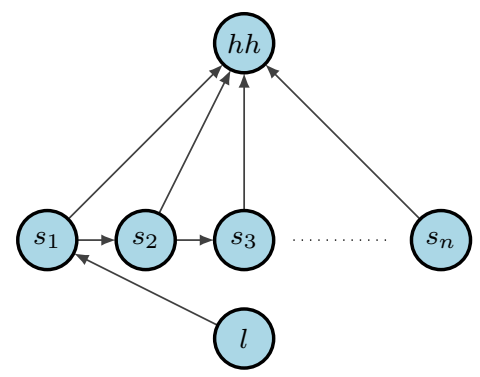

Supply Bottleneck: $\alpha \rightarrow 0, \rho \in(0,1)$

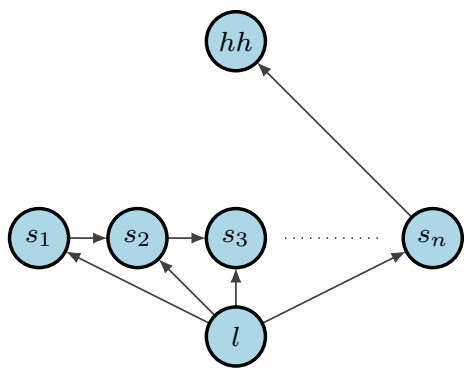

Demand Bottleneck: $\rho \rightarrow 0, \alpha \in(0,1)$

Figure 2: Supply and Demand Bottlenecks

by the most downstream sector: sector $N$. However, all sectors continue to use labor as an input and as such there remain multiple paths by which labor is transformed into consumption. These economies are presented in Figure 2.

Despite the existence of multiple labor-to-consumption paths, in either economy there exists a bottleneck sector. We call a bottleneck sector a sector through which all labor-to-consumption paths must pass. In the limit economy in part (i), even though the household consumes all goods, all labor must first pass through sector 1 . Thus sector 1 serves as a supply-bottleneck. In the limit economy in part (ii), although labor enters the production of every sector, all intermediate inputs are ultimately funneled through sector $N$ en route to consumption. Thus sector $N$ serves as a demand-bottleneck.

The results in Proposition 6 show that in either case, the bottleneck sector is crucial: a distortion to that sector distorts all labor-to-consumption paths. As a result, the bottleneck sector always exhibits the greatest labor wedge influence of all sectors. Moreover, there is a 1-to-1 pass-through of a bottleneck distortion to the aggregate labor wedge. Proposition 6 furthermore states that the labor wedge influence of sectors monotonically decreases as one moves away from the bottleneck sector and this strict ordering is invariant to the aggregate state.

Yet, despite the fact that bottleneck sectors exert the greatest labor wedge influence, a distortion in a bottleneck sector has zero effect on TFP! In either economy, TFP is independent of the bottleneck sector's distortion but not independent of the distortions in all other sectors. This is because a rebated distortion in the bottleneck sector generates zero misallocation.

Thus far we have explored different limits of the hybrid economy. There is, however, an entire continuum of economies within these limits. In Appendix B we consider intermediate values of $(\alpha, \rho) \in(0,1]^{2}$ and examine the comparative statics of labor wedge influence as we move continuously within the extremes. 


\subsection{Lessons from the Simple Networks}

In any generic production network, there are multiple paths through which primary factors may be transformed into final goods. In the absence of distortions, primary factors and intermediate inputs flow through these paths in a way that maximizes productive efficiency in the Diamond and Mirrlees (1971) sense. Misallocation occurs only when distortions alter the flow of inputs through the network in a way that deviates from the optimal allocation. The extent to which inputs may be misallocated along these paths is what lowers productive efficiency and thereby lowers TFP.

The hybrid supply chain economy highlights the general insight that there may be critical bottleneck sectors through which most or all of these paths must pass. A distortion within a bottleneck sector can lead to large movement in the aggregate labor wedge but little to no movement in TFP. This is because a bottleneck distortion disrupts more labor-to-consumption paths and thereby distorts a larger portion of the returns to labor; as a result, it has a disproportionately large effect in the aggregate labor wedge. On the other hand, a bottleneck distortion generates very little misallocation and thereby has a disproportionately small effect on TFP.

\section{A Quantitative Illustration}

We now study a particular quantitative application of our framework: we consider the network amplification of financial frictions during the 2008-09 Financial Crisis. We calibrate the model to the US input-output tables and use the Gilchrist and Zakrajšek (2012) excess bond premia as a proxy for sectoral financial distortions. We then examine the model's aggregate and cross-sectional implications.

The goal of this quantitative exercise is not to explain a significant part of what happened during the 2008-09 Financial Crisis. Instead, it is simply to illustrate how the US input-output structure may have worked to amplify the financial frictions documented by Gilchrist and Zakrajšek (2012) during this period.

This section is organized as follows. In subsection 6.1 we describe the data and our calibration. In subsection 6.2 we produce the model-implied time series for TFP and the aggregate labor wedge. We then focus on the financial crisis and provide a quantification of the amplification generated by the US input-output network in subsection 6.3. Finally, we test the model's ability to match crosssectoral co-movement in subsection 6.4. Supporting figures and analysis are provided in Appendix $\mathrm{C}$ and a detailed description of the data is provided in Appendix D. 


\subsection{The Data and Calibration}

Our primary data sources are the Bureau of Economic Analysis's (BEA) input-output tables. These tables are produced annually from 1997 to 2015 and cover the 3-digit level NAICS industries. For each sector $i$ in year $t$, we observe the following: (i) its total sales, the empirical analogue of $p_{i t} y_{i t}$, (ii) its expenditure on goods produced by sector $j$ in year $t$, the empirical analogue of $p_{j t} x_{i j, t}$, (iii) its labor expenses, the empirical analogue of $w_{i t} \ell_{i t}$ where we let $w_{i t}$ denote the sectoral wage rate, and (iv) the final use of the sector's production, our empirical analogue of $p_{i t} c_{i t}$.

We use this data to construct annual labor and consumption share parameters according to the model's first-order conditions:

$$
\alpha_{i t}=\frac{w_{i t} \ell_{i t}}{w_{i t} \ell_{i t}+\sum_{j} p_{j t} x_{i j, t}}, \text { and } v_{i t}=\frac{p_{i t} c_{i t}}{\sum_{i} p_{i t} c_{i t}} \text { for all } i, t
$$

along with the following input shares:

$$
g_{i j t}=\frac{p_{j t} x_{i j, t}}{\sum_{j} p_{j t} x_{i j, t}}, \text { for all } i, j, t
$$

As these values are relatively stable from year to year, for the rest of our analysis we drop their time subscript and simply use their 2007 values, the year prior to the Great Recession. In Appendix C we document the heterogeneity in these parameters across industries. ${ }^{25}$

A measure of financial distortions: the GZ excess bond premia. In this application we return to the financial frictions economy presented at the end of Section 3 in which we interpret the distortions as the result of working capital constraints. We use the sector-level excess bond premia of Gilchrist and Zakrajšek (2012) as our measure of financial frictions.

Gilchrist and Zakrajšek (2012) construct their "GZ credit spread" as follows. First, the authors take individual fixed-income securities and discount their promised cash-flows according to zerocoupon US Treasury yields. This procedure delivers an overall credit spread for each security: the yield of the corporate bond over a hypothetical Treasury security with exactly the same cash flows.

The "GZ excess bond premium" for each security is then constructed as the portion of the overall credit spread that cannot be accounted for by individual predictors of default nor bond-specific characteristics. Specifically, the authors regress their credits spreads on a firm-specific measure of expected default and a vector of bond-specific characteristics; the residual of this regression is the GZ excess bond premium.

\footnotetext{
${ }^{25}$ See "Heterogeneity in Labor, Intermediate Good, and Consumption Shares" in Appendix C. We report the top 20 industries in our sample with the greatest labor shares of expenditure $\alpha_{i}$, the top 20 industries with the greatest intermediate good shares of expenditure $1-\alpha_{i}$, and the top 20 industries with the greatest shares in final uses, $v_{i}$.
} 
A word should be said on how the authors measure a firm's probability of default at each point in time. The authors construct a distance to default measure based on the Merton (1974) model. The equity of the firm can be viewed as a call option on the underlying value of the firm with a strike price equal to the face value of the firm's debt. Assuming that the firm's value follows a geometric Brownian motion, one may then apply the Black-Scholes-Merton option-pricing formula and calculate distance to default with observable asset price data. As Gilchrist and Zakrajšek (2012) state, "the distance to default should summarize all available information regarding the risk of default, according to the Merton model."

We use sector-level indices of the GZ excess bond premia, generously provided to us by the authors, as our financial frictions measure. This measure has several virtues that make it ideal for our application. By orthogonalizing out default probabilities, these premia are meant to capture time- and sector-varying changes in the compensation demanded by the financial sector in providing credit - above and beyond expected losses. Gilchrist and Zakrajšek (2012) find that adverse shocks to highly leveraged financial intermediaries during the 2008-09 crisis led to sharp increases in their measure. This evidence is consistent with the notion that the GZ excess bond premium reflects a reduction in the risk-bearing capacity of the financial sector, and as a result, an increase in the cost of credit extended to firms. We refer the reader to their paper for more details.

For each sector $i$ in year $t$, we denote the GZ sectoral excess bond premia by $r_{i t}^{G Z}$. We interpret this premia as the intra-period interest rate faced by sector $i$ in period $t$, i.e. the sector's cost of borrowing. As shown in Proposition 2, sectoral distortions in the model may be related to the cost of borrowing as follows:

$$
\phi_{i t}=\frac{1}{1+r_{i t}^{G Z}}
$$

We thereby construct our baseline measure of sectoral distortions according to equation (31) and hereinafter refer to these as the "GZ distortions."

Returns to Scale (or Factor Income Shares). Finally, our baseline model makes the simplifying assumption that there are constant returns-to-scale to production and that labor is the only primary factor. In our quantitative analysis, we relax this assumption and allow for sector-specific fixed factors in a reduced-form way. We modify the production function as follows:

$$
y_{i t}=z_{i, t}\left(\ell_{i t}^{\alpha_{i}} x_{i t}^{1-\alpha_{i}}\right)^{\eta_{i}}
$$

One may either interpret the new sector-specific parameter $\eta_{i} \in(0,1]$ as the sector's decreasing returns-to-scale parameter or equivalently, in a model with fixed factors, $\eta_{i}$ is the share of total income accrued to labor and intermediates. In this latter interpretation, $1-\eta_{i}$ is the share of total income accrued to all other fixed factors, e.g. physical capital or land. In either case, the labor 
share of total income for a sector is $\alpha_{i} \eta_{i}$.

We assume $\eta_{i}$ may be heterogeneous across sectors but constant over time. The question then becomes how one may calibrate this parameter. In each sector we observe total expenditure on inputs and gross sales each period. Therefore, we may compute each sector's expenditure-to-sales ratio, which we denote here by $\varepsilon_{i t}$ :

$$
\varepsilon_{i t} \equiv \frac{w_{i t} \ell_{i t}+\sum_{j} p_{j t} x_{i j, t}}{p_{i t} y_{i t}}=\phi_{i t} \eta_{i}
$$

In a frictionless economy with Cobb-Douglas technologies, this ratio is constant over time. With distortions, this ratio satisfies $\varepsilon_{i t}=\phi_{i t} \eta_{i}$. Unfortunately, we have no data to separately identify $\eta_{i}$ from $\phi_{i t}{ }^{26}$

Overcoming this limitation is rather difficult; we take one approach. We exploit the fact that in each sector $\varepsilon_{i t}$ varies over time while $\eta_{i}$ does not; we believe the latter assumption is reasonable at least within a short time span like the financial crisis. We then construct the entire set of values for $\eta_{i}$ consistent with both the data in our sample and the model. We denote $\eta_{i} \equiv \max _{t} \varepsilon_{i t}$ as the greatest value of the expenditure-to-sales ratio observed for sector $i$ in our sample period. Provided that we restrict $\phi_{i t} \in(0,1], \eta_{i}$ must lie in the closed set

$$
\eta_{i} \in\left[\eta_{i}, 1\right]
$$

Why is this the admissible set? First, if $\phi_{i t} \leq 1$, then $\underline{\eta}_{i}$ is the lowest possible value for $\eta_{i}$. At the other extreme, the highest possible value is 1 , as in the case of CRS technology and no other primary factors. ${ }^{27}$

For all subsequent quantitative analysis we provide results at both extremes: for $\eta_{i}=\eta_{i}$ and for $\eta_{i}=1$. We refer to the former as the lowest possible decreasing returns-to-scale (DRS) model and to the latter as the constant returns-to-scale (CRS) model.

Measures of Sectoral and Aggregate Quantities. While not necessary for our calibration, we construct sectoral quantities using sectoral prices provided by the the BEA. The BEA provides producer price indices for each sector $i$ in year $t$, the empirical analogues of $p_{i t}$. This data allows us to construct output and consumption (final use) quantities, $y_{i t}$ and $c_{i t}$, for all $i, t$. We furthermore construct individual input quantities $x_{i j, t}$ for all $i, j, t$ and use these to construct each sector's

\footnotetext{
${ }^{26}$ Concerning this particular issue Jones (2013) writes, "there is a fundamental identification problem: we see data on observed intermediate good shares and we do not know how to decompose that data into distortions and differences in technologies."

${ }^{27}$ By setting $\underline{\eta_{i}}$ to the maximum observation of (33), we implicitly assume that there is at least one year in our sample in which sector $i$ faces no distortions; in this year, $\phi_{i t}=1$. In all other years, $\phi_{i t}<1$. Clearly it could also be the case that the sector always faces distortions in all periods, that is, $\phi_{i t}<1$ in all years $t$; in this case $\eta_{i}$ would be bounded away from $\underline{\eta_{i}}$ and would fall in the interior of the set in 34 .
} 


\begin{tabular}{|c|c|c|}
\hline & Mean & Median \\
\hline std dev $\left(\Delta \log \phi_{i t}\right)$ & 0.014 & 0.013 \\
\hline $\operatorname{corr}\left(\Delta \log \phi_{i t}, \Delta \log y_{i t}\right)$ & 0.313 & 0.289 \\
\hline $\operatorname{corr}\left(\Delta \log \phi_{i t}, \Delta \log l_{i t}\right)$ & 0.348 & 0.394 \\
\hline $\operatorname{corr}\left(\Delta \log \phi_{i t}, \Delta \log x_{i t}\right)$ & 0.277 & 0.336 \\
\hline $\operatorname{corr}\left(\Delta \log \phi_{i t}, \Delta \log \epsilon_{i t}\right)$ & 0.195 & 0.225 \\
\hline
\end{tabular}

Table 1: Sectoral Distortions Cross-Sectional means of within-sector correlations

basket of intermediate goods, $x_{i, t}$, according to (2). We obtain measurements of labor in each sector, $\ell_{i t}$, calculated as hours worked from the Bureau of Labor Statistics (BLS). In Appendix C, we document their within-sector time-series properties. ${ }^{28}$

We construct aggregate consumption $C_{t}$ by aggregating over $c_{i t}$ according to the Cobb Douglas consumption basket in (4). Therefore, our constructed series for $c_{i t}$ and $C_{t}$ actually correspond to sectoral and aggregate final use quantities in the data rather than consumption. Aggregate labor $L_{t}$ is calculated as the sum of hours worked.

None of the constructed quantities described here are ever used in calibrating the model; we construct them simply for diagnostic purposes at the sectoral level. The aggregates $C_{t}$ and $L_{t}$ are used only in computing our data analogue of the labor wedge.

\section{Time-Series and Cross-Sectional Properties of the GZ Distortions}

We now consider both the within-sector time-series and the within-year cross-sectional properties of the sectoral GZ distortions series.

Within-sector time-series. Within each sector we calculate the standard deviation over time of $\Delta \log \phi_{i t}$ and report the cross-sectional mean and median in the first row of Table 1 . The mean standard deviation for $\Delta \log \phi_{i t}$ over our sample is around $1.4 \%$. We furthermore compute the within-sector time-series correlations between log changes in GZ distortions and in the constructed series of output, hours, and inputs; we report their cross-sectional means and medians in the second to fourth rows of Table 1. Typically, within a sector there is positive co-movement between $\log$ changes in distortions and log changes in output (31\%), hours (35\%), and inputs (28\%).

Within-year cross-section. For each year we compute the cross-sectional mean of the log changes in GZ distortions, output, and hours; we plot their time series over our sample period in the left panel of Figure 3. The cross sectional mean of $\Delta \log \phi_{i t}$ is indicated by the solid blue line (measured against the right axis); the cross-sectional means of $\Delta \log y_{i t}$ and $\Delta \log \ell_{i t}$ are plotted by the dashed dark red line and the dotted grey line, respectively (both measured against the left axis). The

\footnotetext{
${ }^{28}$ See "Time-Series Statistics for Constructed Quantities" in Appendix C.
} 
correlations of these series are fairly high: log changes in distortions are $70 \%$ correlated with output and $74 \%$ with hours. Comparing these to the numbers reported in Table 1, movements in distortions appear to be more strongly correlated with movements in hours and output at the aggregate level than at the sectoral level. Furthermore, it is clear that our data picks up two recessions: the dotcom-crash of 2001 and the financial crisis of 2008-2009.
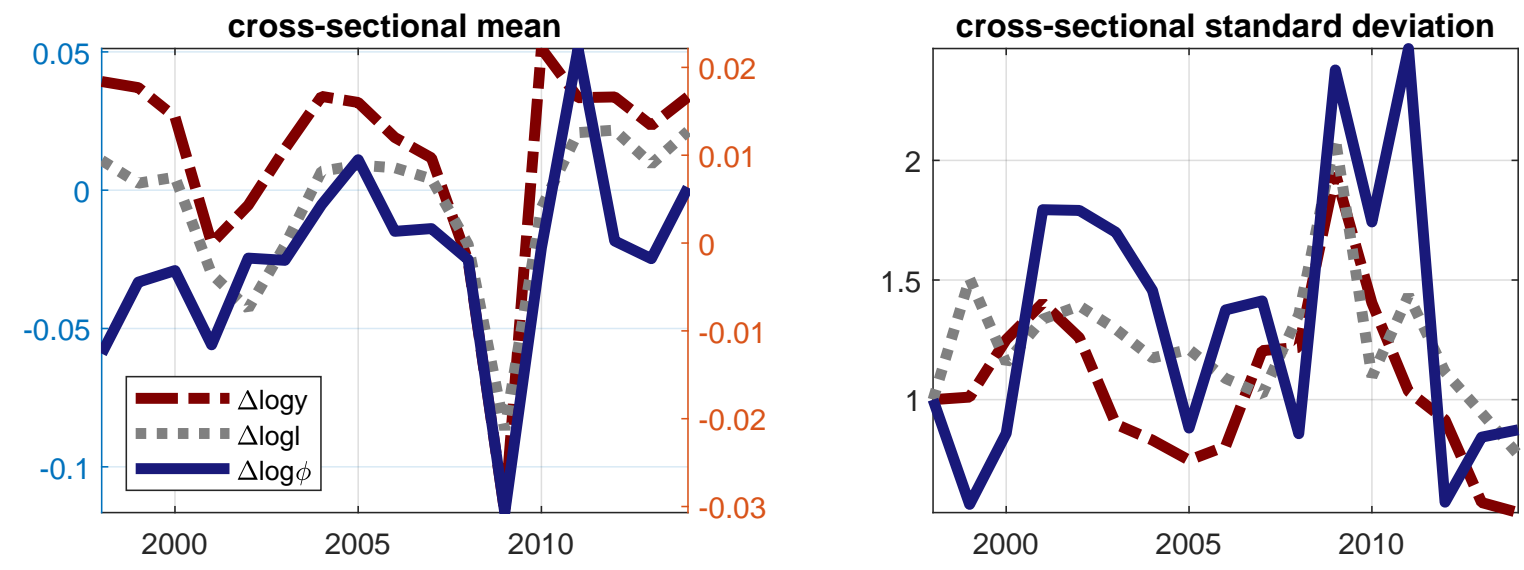

Figure 3: Left panel: Time series for cross-sectional means of $\Delta \log y_{i t}$ (left axis), $\Delta \log \ell_{i t}$ (left axis), and $\Delta \log \phi_{i t}$ (right axis). Right panel: Time series for cross-sectional standard deviations of $\Delta \log y_{i t}, \Delta \log \ell_{i t}, \Delta \log \phi_{i t}$; all series are normalized to 1 in the first year.

For each year we moreover compute the cross-sectional standard deviations in log changes in GZ distortions, output, and hours and plot their time series in the right panel of Figure 3. For this figure we normalize the standard deviations in the first year of our sample to one in order to compare movements. We find that in both the 2001 and 2008-2009 periods the cross-sectional standard deviations increase relative to previous years.

Finally, we examine these relationships at the individual industry level in Appendix C. We focus on eight specific industries: utilities, construction, durable manufacturing sectors (wood, fabricated metals, computers, electricals, and motor vehicles) and a non-durable manufacturing sector: plastics \& rubber products. For these sectors, as with most other sectors, the GZ distortion co-moves positively with sectoral output. ${ }^{29}$

Correlation of the GZ Measure \& Expenditure-to-Sales ratios. The expenditure-to-sales ratio in (33) varies over time for each sector in our sample. According to the model and provided that $\eta_{i}$ remains constant, any time-series movement in $\varepsilon_{i t}$ is a direct consequence of movement in the sectoral wedge, $\phi_{i t}$. To the extent that financial frictions are only one out of a myriad of all possible distortions, variation in the GZ distortions constructed in (31) cannot possibly capture all

\footnotetext{
${ }^{29}$ See "Time-Series Properties of Distortions for Individual Industries" in Appendix C.
} 
variation in expenditure-to-sales ratios seen in the data. However, one may ask how much of the observed variation it does capture. We thus examine the within-sector time-series correlation of these objects as well as the time-series correlation of their cross-sectional means.

Within-sector time-series. Within each sector we compute the time-series correlation between $\Delta \log \phi_{i t}$ and $\Delta \log \varepsilon_{i t}$; we report their cross-sectional means and medians in the final row of Table 1. Typically within a sector there is a positive co-movement between log changes in its GZ distortions and expenditure-to-sales ratios, with mean and median correlations of $19.5 \%$ and $22.5 \%$, respectively.

Within-year cross-section. For each year we compute the cross-sectional mean of the log changes in distortions and expenditure-to-sales ratios; we plot their time series over our sample period in the left panel of Figure 4. The cross sectional mean of $\Delta \log \phi_{i t}$ is given by the solid blue line and the cross-sectional mean of $\Delta \log \varepsilon_{i t}$ by the dashed dark red line. These two series are highly correlated at $73 \%$. Again, comparing this to the numbers reported in Table 1, movements in distortions appear to be more strongly correlated with movements in expenditure shares at the aggregate level than at the sectoral level.
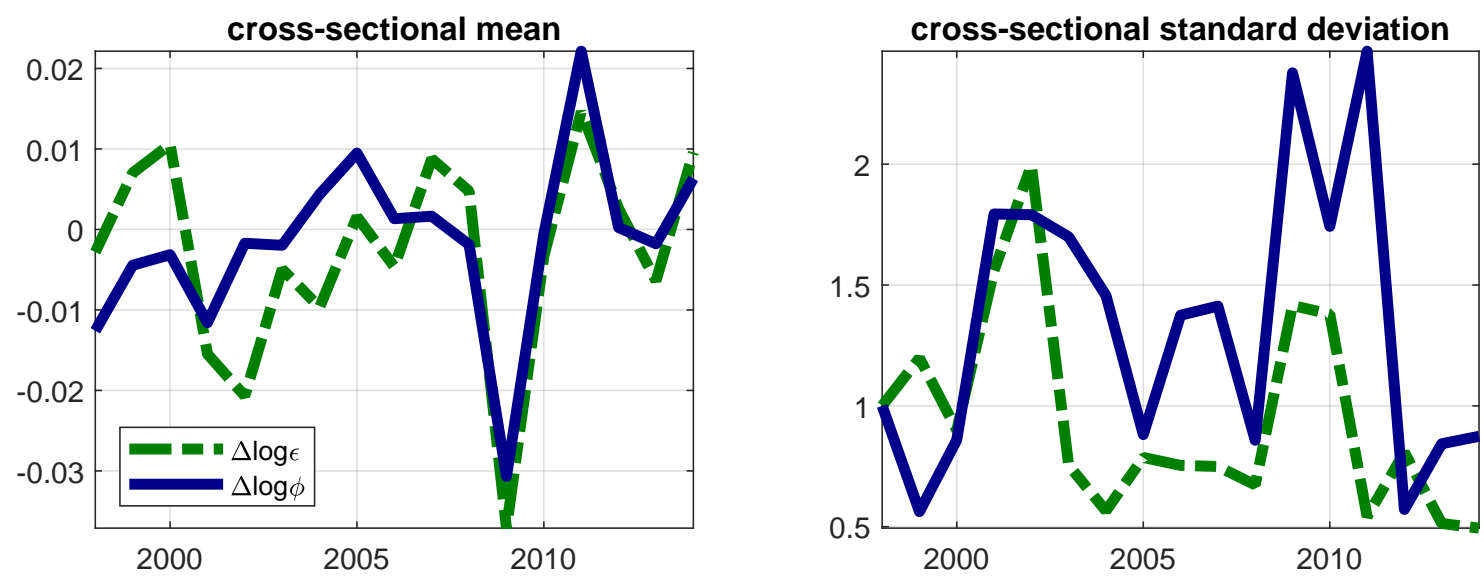

Figure 4: Left panel: Time series for cross-sectional means of $\Delta \log \phi_{i t}$ and $\Delta \log \varepsilon_{i t}$. Right panel: Time series for cross-sectional standard deviations of $\Delta \log \phi_{i t}$ and $\Delta \log \varepsilon_{i t}$; both series are normalized to 1 in the first year.

For each year we moreover compute the cross-sectional standard deviations of log changes in distortions and expenditure shares and plot their time series in the right panel of Figure 4; we again normalize these standard deviations to one for the first year of our sample. In both recessions, the cross-sectional standard deviations of these measures increase relative to previous years. We again take a closer look at these relationships at the individual industry level in Appendix C. 


\subsection{Model Implications for Aggregate Time-Series Movements}

We now evaluate the aggregate network effects of financial frictions through the lens of our calibrated model. We abstract from TFP shocks and allow only for movements in the sectoral GZ distortions. We construct the model-implied time series for TFP and the labor wedge. Figures 5 and 6 plot the model-implied movements in $\log T F P(s)$ and $\log \Lambda(s)$, respectively, over the sample period (1998-2015). In both figures our results for the lowest DRS model $\left(\eta_{i}=\eta_{i}, \forall i\right)$ are indicated by the dashed blue line and those for the the CRS model $\left(\eta_{i}=1, \forall i\right)$ are in plotted in solid red. In either figure, it is clear that the two models give similar results: the CRS model is simply an amplified version of the lowest DRS model.

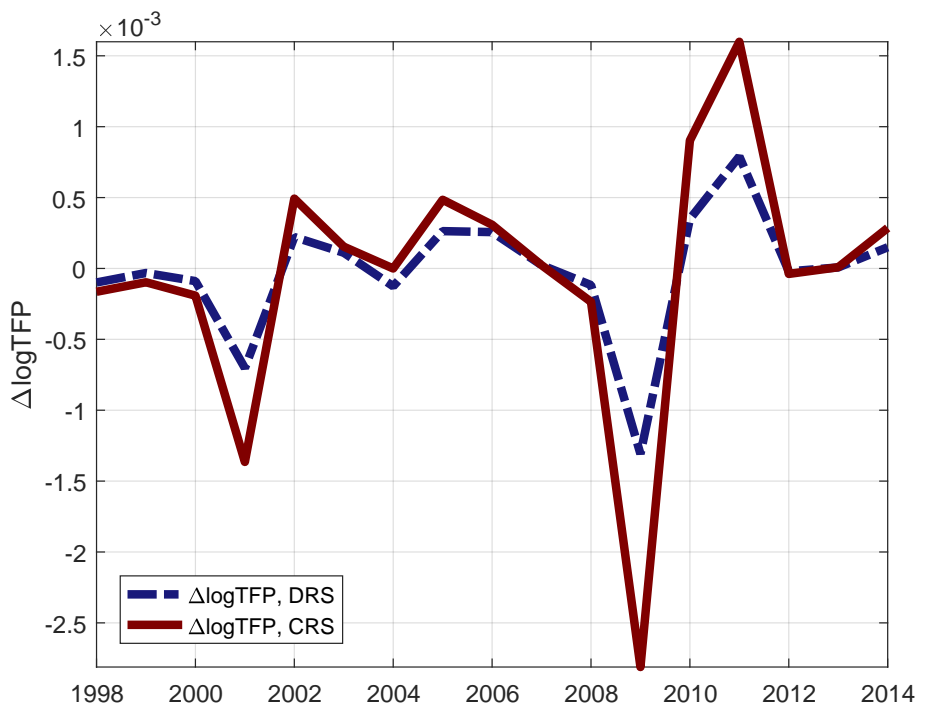

Figure 5: Time Series for model-generated $\Delta \log T F P$

In Figure 6 we moreover superimpose the percentage change in the aggregate labor wedge computed in our data: this is plotted by the dotted teal line. ${ }^{30}$ Our model implies similar movements in the labor wedge to those computed in the data; in fact, the correlation between the model-implied labor wedge and its data analogue is 0.71 in the lowest-DRS model and 0.69 in the CRS model.

From 2008 to 2009, the calibrated model with GZ shocks produces a 5.91 percentage fall in the labor wedge in the CRS model and a 4.93 percentage fall in the labor wedge in the DRS model. The labor wedge computed in our data experiences a 6.71 percentage fall during this period. Therefore, the calibrated network model with GZ shocks can account for $73-88 \%$ of the fall in the labor wedge

\footnotetext{
${ }^{30}$ We construct the data counterpart of the aggregate labor wedge according to its definition (13) with homothetic preferences specified in (35). Together these imply an aggregate labor wedge given by

$$
\log \Lambda(s)=(1+\epsilon) \log L(s)-(1-\gamma) \log C(s)
$$

where $C$ and $L$ are aggregate consumption and hours computed in our data. Finally, we set $\epsilon=0.5$ and $\gamma=0.1$ as our baseline parameterization.
} 


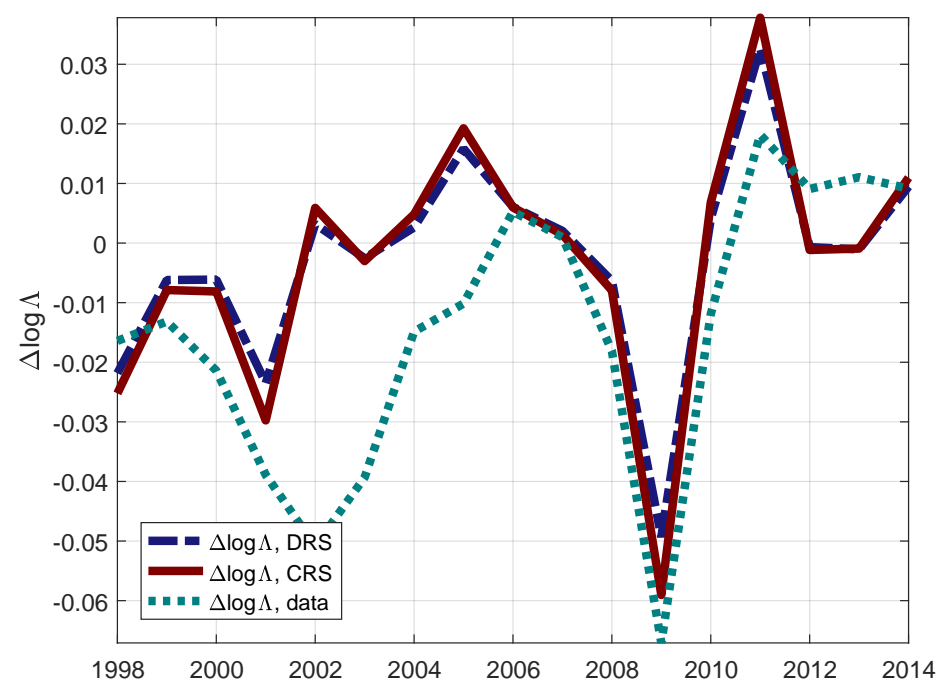

Figure 6: Time Series for model-generated $\Delta \log \Lambda$

computed in our data from 2008-2009.

The two recessions in our sample, the 2001 Dotcom bust and the 2008-09 Financial Crisis, are evident in both Figures 5 and 6. However, it's clear from comparing the scales of these figures that the calibrated model generates movement in the aggregate labor wedge that is an order of magnitude larger than the model-generated movement in TFP. For example, from 2008-2009 the calibrated model produces only a .28 percentage fall in TFP in the CRS model and a .13 percentage fall in TFP in the DRS model - these are minuscule compared to the 5.91 and 4.93 percentage drops in the model-implied labor wedges cited above.

This is consistent with our theory. As we have abstracted entirely from productivity shocks, the driving forces in the calibrated model are the GZ distortions alone. From Theorem 2 we know that near efficiency, sectoral distortions generate first-order movement in the labor wedge, but have only higher-order effects on TFP. As a result, any movement in the model-generated TFP is a result of misallocation alone.

Finally, we translate the model-implied movements in TFP and the labor wedge into modelimplied time-series for aggregate consumption and labor. Recall that Theorem 1 holds for any specification of the utility functions $U, V$ that satisfy the typical regularity conditions. However, in order to solve for aggregate consumption and labor, one must specify preferences. For this exercise we make the standard assumption that preferences are homothetic. We let:

$$
U(C) \equiv \frac{C^{1-\gamma}}{1-\gamma} \text { and } V(L) \equiv \frac{L^{1+\epsilon}}{1+\epsilon} .
$$

with $\gamma \geq 0$ and $\epsilon>0$. Given the static nature of the model, the parameter $\gamma$ governs the wealth 
effect on labor supply while $\epsilon$ controls the substitution effect: $\epsilon$ is the inverse Frisch elasticity. ${ }^{31}$ With this specification we solve explicitly for equilibrium consumption and labor; the solution is provided in Lemma 4 in Appendix A.
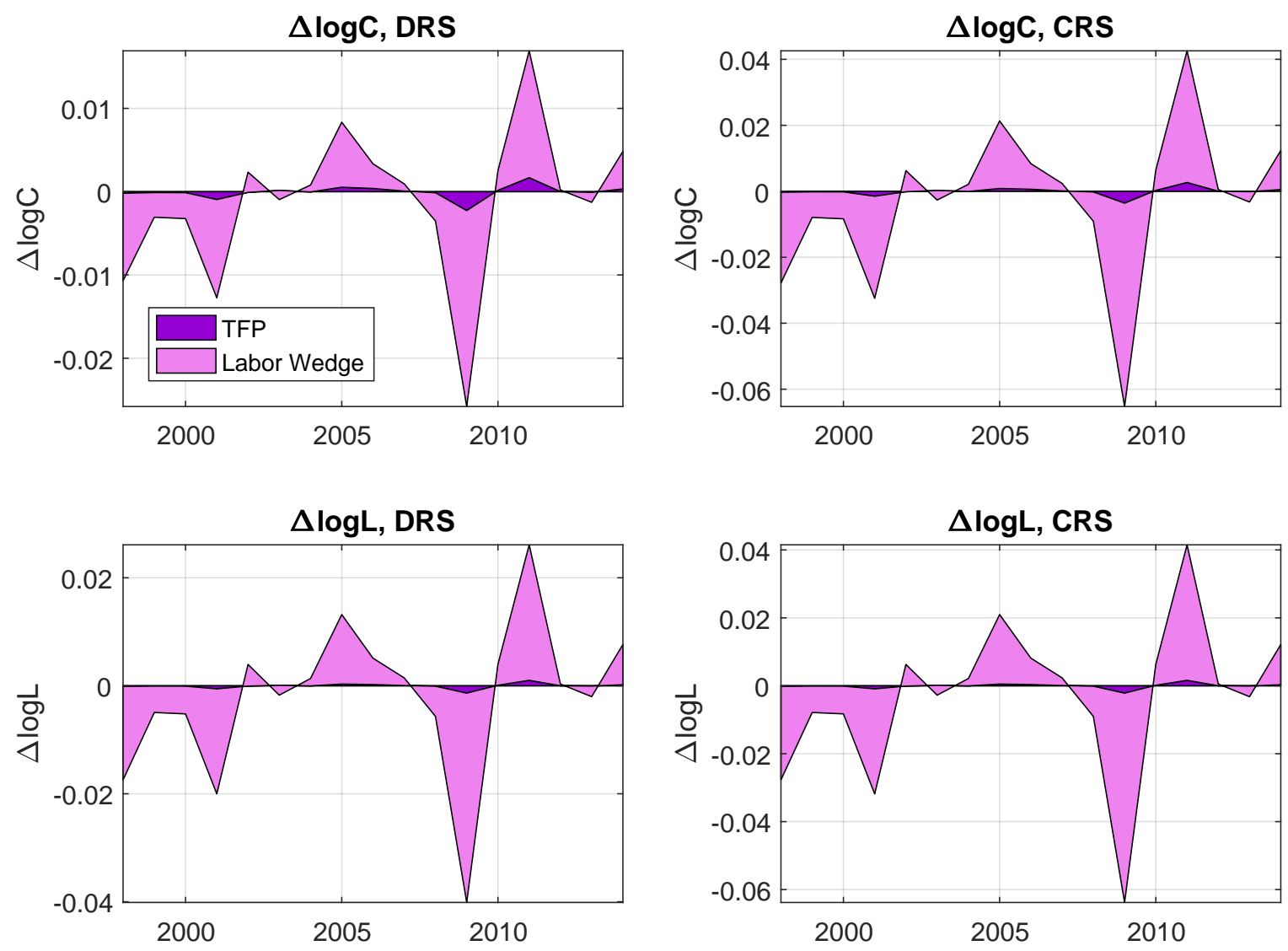

Figure 7: Decomposition of aggregate consumption and labor between TFP and the aggregate labor wedge. Consumption in the DRS model (top left panel); consumption in the CRS model (top right panel); labor in the DRS model (bottom left panel); labor in the CRS model (bottom right panel).

We plot the model-implied movements for $\log C$ and $\log L$ over our sample period in Figure 7 for $\epsilon=0.5$ and $\gamma=0.1 .^{32}$ Percentage movements in aggregate consumption are plotted in the top panel; percentage movements in aggregate labor are plotted in the bottom panel. The left panel plots these series for the DRS model, while those for the CRS model are plotted on the right.

In this figure we decompose the model-predicted consumption and labor into the portions driven by the two aggregate channels. The light shaded region represents variation in consumption or

\footnotetext{
${ }^{31}$ Risk aversion and intertemporal substitution play no role in our setting because all idiosyncratic risk is insurable and the model is static. Therefore, $\gamma$ controls only the elasticity of labor supply to income for a given wage. Note that when $\gamma=0$ there is no income effect, similar to GHH preferences, see Greenwood et al. (1988).

${ }^{32}$ This parameterization is in line Hall (2009); setting $\epsilon=0.5$ implies a Frisch elasticity of labor supply of 2 . Setting $\gamma=0.1$ essentially minimizes any wealth effect, e.g. as in Greenwood et al. (1988).
} 
labor due to the labor wedge, while the darker purple region represents variation driven by TFP. Consistent with the theory we find that the labor wedge is the quantitatively stronger channel of the two as it explains the bulk of the movement in consumption and labor. In particular, in the DRS model $93 \%$ of the drop in consumption and $97 \%$ of the fall in labor during the recession is due to the aggregate labor wedge; the corresponding numbers in the CRS model are $96 \%$ and $97 \%$.

\subsection{Network Amplification during the Financial Crisis}

We next focus only on the years surrounding the financial crisis and ask: what is the role played by the input-output network in amplifying the financial frictions documented by Gilchrist and Zakrajšek (2012) during the crisis?

\subsubsection{The Network Multiplier during the Financial Crisis}

In order to answer this question, we must disentangle the aggregate network effect from the impact of the distortions themselves. We thus compare the predictions of our calibrated model - calibrated to the US input-output network - to an otherwise equivalent model without any input-output linkages. We call the latter the "equivalent horizontal economy" and construct it as follows. First, each sectors' share in the final consumption basket is kept equal to that in the calibrated network economy. We then remove all input-output linkages by taking the limit as the intermediate input share of expenditures goes to zero in all sectors, or equivalently, as the labor share of expenditures $\alpha_{i}$ approaches one. With the equivalent horizontal economy so constructed, we define our measure of network amplification as follows.

Definition 2. Take any input-output economy and its equivalent horizontal economy; in each economy compute the aggregate effect of a symmetric distortion across all sectors. The labor wedge network multiplier is the ratio between the percentage change in the labor wedge generated by the input-output economy and that generated by its equivalent horizontal economy.

Because the same vector of symmetric shocks are fed into the two economies, the labor wedge network multiplier is a function of the network structure alone. One may likewise define the network multiplier for TFP; however, as discussed in Section 5, if distortions are symmetric then TFP is unchanged in any horizontal economy. As a result, the TFP network multiplier tends to infinity.

We simulate the two alternative economies for 2008-2009 and report the results in Table 2. For symmetric distortions, we simply use the cross-sectional means of the GZ distortions during this period. The first row in Table 2 reports the percentage change in TFP and the percentage change

in the aggregate labor wedge between 2008 and 2009 for the equivalent horizontal economy. The second row reports the percentage movement in these aggregates for the calibrated input-output 


\begin{tabular}{|l|c|c|c|c|}
\hline & \multicolumn{2}{|c|}{$\Delta \log T F P$} & \multicolumn{2}{c|}{$\Delta \log \Lambda$} \\
\hline Returns-to-Scale: & DRS & CRS & DRS & CRS \\
\hline \hline Horizontal Network, Symmetric $\phi$ & 0 & 0 & -.0307 & -.0307 \\
\hline US-IO Network, Symmetric $\phi$ & -.0012 & -.0027 & -.0523 & -.0650 \\
\hline US-IO Network, Asymmetric $\phi$ & -.0013 & -.0028 & -.0493 & -.0591 \\
\hline Network Multiplier & $\infty$ & $\infty$ & 1.70 & 2.12 \\
\hline
\end{tabular}

Table 2: The Network Multiplier during the Financial Crisis (2008-2009)

economy. To calculate the network multiplier for the labor wedge, one simply divides the number in the second row by that in the first; the result is reported in the fourth row of Table 2. The labor wedge network multiplier is 1.70 for the DRS model and 2.12 in the CRS model.

The network multiplier measures the network amplification of symmetric distortions in order to isolate the role of the network structure. However, movements in the GZ distortions feature significant heterogeneity across sectors; this asymmetry may lead to further movements in aggregates. Thus, in the third row of Table 2 we report the calibrated network model's aggregate responses to the actual asymmetric distribution of GZ shocks.
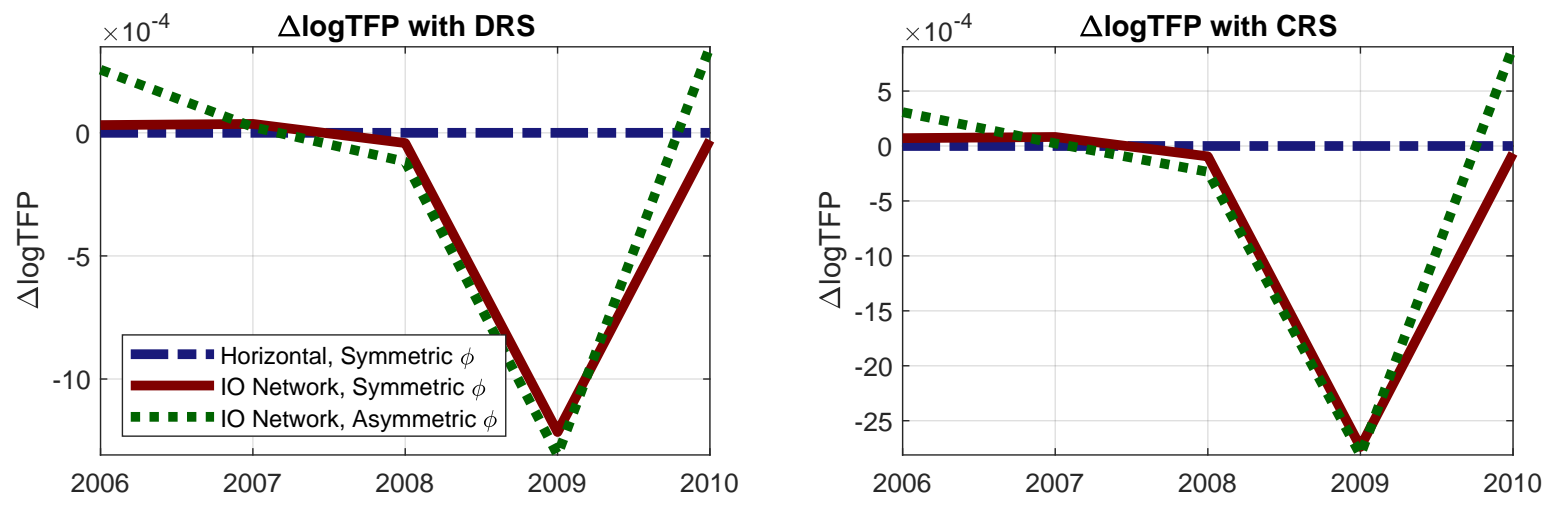

Figure 8: Network amplification of TFP during the financial crisis

A visual interpretation of the network amplification generated by the model is provided in Figures 8 and 9 for the years surrounding the crisis (2006-2010). Figure 8 plots the percentage change in TFP generated by the model for the three cases discussed above: the horizontal economy with symmetric shocks (indicated by the dashed blue line), the calibrated US input-output economy with symmetric shocks (the solid dark red line), and the calibrated network with asymmetric shocks (the dotted dark green line); Figure 9 provides the analogous plots for the model-implied labor wedge. In either figure, the left panel presents these series for the lowest DRS model, while the right panel presents these for the CRS model.

To visualize the amplification generated by the network, one may simply compare the calibrated 

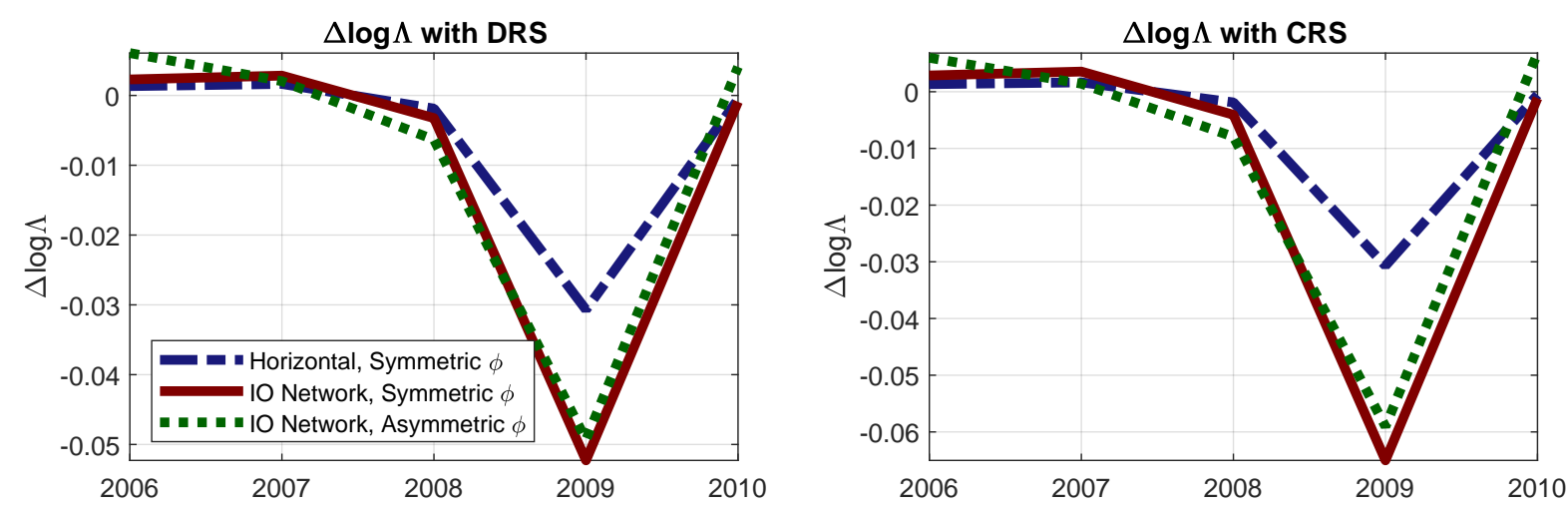

Figure 9: Network amplification of the labor wedge during the financial crisis

network economy with symmetric shocks to the horizontal economy with symmetric shock. In both figures, the input-output network appears to generate considerable amplification. ${ }^{33}$

\subsubsection{Back-of-the-Envelope Calculation for the Network Multiplier}

Our results in Table 2 indicate that the network multiplier for the labor wedge during the financial crisis is roughly 2 . We now relate this number to a certain simple back-of-the-envelope calculation based on the theory.

In particular, we use part (ii) of Theorem 2 to obtain a closed-form approximation for the labor wedge network multiplier in the CRS economy. Suppose distortions are small and symmetric: $d \log \phi_{i}=d \log \bar{\phi}$ for all $i \in I$. Then, applying equation (24), we have:

$$
\left.d \log \Lambda_{I O}\right|_{\phi=\mathbf{1}} \approx\left(\mathbf{v}^{\prime} \mathbf{B}_{\mathbf{I O}} \mathbf{1}\right) d \log \bar{\phi}
$$

where $\mathbf{B}_{\mathbf{I O}}$ is the Leontief inverse matrix of the calibrated U.S. input-output network. On the other hand in the horizontal economy, application of equation (24) yields:

$$
\left.d \log \Lambda_{h o r}\right|_{\phi=1} \approx d \log \bar{\phi}
$$

where $\mathbf{B}_{\text {hor }}=\mathbb{I}_{N}$, thus $\mathbf{v}^{\prime} \mathbf{1}=1$. Note here that we could have used any horizontal economy for this exercise: the vector of consumption shares $\mathbf{v}$ does not matter as long as shocks are symmetric.

The labor wedge network multiplier may thereby be approximated by the ratio of (36) to (37):

$$
\frac{\left.d \log \Lambda_{I O}\right|_{\phi=1}}{\left.d \log \Lambda_{h o r}\right|_{\phi=1}} \approx \mathbf{v}^{\prime} \mathbf{B}_{\mathbf{I O}} \mathbf{1} .
$$

\footnotetext{
${ }^{33}$ In Figure 8, TFP in the horizontal economy is unresponsive to movement in symmetric distortions; this coincides with the theory.
} 
In this approximation the symmetric shocks cancel out and we are left with the sum of the elements of the influence vector. Equation (38) thereby gives us a simple approximation for the labor wedge network multiplier in terms of the underlying structural parameters of the model.

We may use an identical method, this time based on Corollary 2, to obtain a sufficient statistic for the labor wedge network multiplier. For small, symmetric distortions,

$$
\left.d \log \Lambda_{I O}\right|_{\phi=\mathbf{1}} \approx\left(\sum_{i \in I} \lambda_{i}\right) d \log \bar{\phi},
$$

where $\sum_{i \in I} \lambda_{i}$ is the sum of the Domar weights. Applying this approximation to any horizontal economy yields $\left.d \log \Lambda_{h o r}\right|_{\phi=1} \approx d \log \phi$. In any economy in which there is no intermediate good trade, the sum of the Domar weights is equal to 1 regardless of the distribution of consumption shares.

Taking the ratio of these two objects, we find that the labor wedge network multiplier is approximately equal to the sum of the Domar weights:

$$
\frac{\left.d \log \Lambda_{I O}\right|_{\phi=1}}{\left.d \log \Lambda_{h o r}\right|_{\phi=1}} \approx \sum_{i \in I} \lambda_{i}=\frac{\sum_{i} p_{i} y_{i}}{C} .
$$

The sum of the Domar weights is moreover equal to the ratio of gross sales to consumption. In our data this ratio is roughly equal to 2 and thereby coincides with our full model's estimates of the network multiplier.

In Appendix $\mathrm{C}$ we show how the above analysis relates to another, distinct back-of-the-envelope calculation for the network multiplier presented in Jones (2011) and Jones (2013). ${ }^{34}$

\subsection{Implications for Cross-Sectoral Correlations}

We have thus far examined the aggregate implications of the input-output network with GZ distortions. However, any input-output model also has implications for the cross-section. We now explore whether the calibrated network model with GZ distortions can help explain the cross-sectional comovement observed in sectoral sales.

Sectoral sales are directly observable in the BEA data. We denote the sales in sector $i$ in year $t$ by $m_{i t}=p_{i t} y_{i t}$ and the variance-covariance matrix of log sales by $\Sigma_{\text {data }}^{m}$. The latter is a square matrix in which the elements of the diagonal report the time-series variation of log sales in each industry, while the off-diagonal elements report the time-series covariance of log sales across sector-pairs.

Our goal is to see whether the calibrated network model generates similar covariation across

\footnotetext{
${ }^{34}$ See "Relation to the network multiplier in Jones (2011) and Jones (2013)" in Appendix C.
} 
sector-pairs. We first solve for the equilibrium sales vector implied by the model; this is presented below for the CRS economy. ${ }^{35}$

Proposition 7. Let $m_{i}(s)$ denote the equilibrium sales in sector $i$ and $\mathbf{m}(s) \equiv \operatorname{diag}(\mathbf{p}(s)) \mathbf{y}(s)$ the equilibrium vector of sales. Equilibrium sectoral sales are linear in aggregate labor

$$
\mathbf{m}(s)=\Omega(\phi(s)) L(s)
$$

where $\Omega: \mathbb{R}_{+}^{N} \rightarrow \mathbb{R}_{+}^{N}$ is an $N \times 1$ vector function given by

$$
\Omega(\phi(s)) \equiv\left[\mathbb{I}_{N}-(\operatorname{diag}(\mathbf{1}-\alpha) \mathbf{G})^{\prime} \operatorname{diag}(\phi(s))-\mathbf{v} \mathbf{1}^{\prime}\left(\mathbb{I}_{N}-\operatorname{diag}(\phi(s))\right)\right]^{-1} \mathbf{v}
$$

Equilibrium sectoral sales are thereby linear in aggregate labor. We apply this characterization to the calibrated network economy and its equivalent horizontal economy. In each economy we feed in the sectoral GZ distortions series and use Proposition 7 to compute the model-implied equilibrium sales vector for each year. ${ }^{36}$ From the model-generated sales data we compute the variance-covariance matrix of log sales for each economy as we did in the data. Because the model generally produces much less variation in sectoral sales relative to that observed in actual sales, we normalize the model-implied variance-covariance matrices so that they have the same crosssectional mean variance as in data. We denote the normalized variance-covariance matrices of log sales for the calibrated input-output network economy and the equivalent horizontal economy by $\Sigma_{I O}^{m}$ and $\Sigma_{h o r}^{m}$, respectively. ${ }^{37}$

\subsubsection{Distance comparison.}

Does the calibrated network model with GZ distortions do a better job than the horizontal model with GZ distortions in matching the sectoral covariances in log sales found in the data? In order to answer which of the two model-generated variance-covariance matrices is "closer to" the true one measured in the data, we need some norm for measuring the distance between two matrices. While there are many norms one might consider; we use the Frobenius norm. The Frobenius norm, or distance, between two matrices $A$ and $B$ is defined as the square root of the sum of the squares of

\footnotetext{
${ }^{35}$ Appendix A contains the proof of Proposition 7 for the CRS economy. See Appendix E for the corresponding result and proof for the DRS economy.

${ }^{36}$ For this exercise we care about the covariance structure implied by the model; for this reason we use the measured asymmetric distribution of GZ distortions rather than the symmetric one.

${ }^{37}$ Formally, we normalize so that the cross-sectional mean of the diagonal elements of $\Sigma_{\text {hor }}^{m}$ and $\Sigma_{\text {net }}^{m}$ are both equal to the cross-sectional mean of the diagonal elements $\Sigma_{\text {data }}^{m}$.
} 
the difference in elements:

$$
d(A, B)=\|A-B\| \equiv \sqrt{\sum_{i} \sum_{j}\left(a_{i j}-b_{i j}\right)^{2}}
$$

This norm seems natural: it is the Euclidean norm for vectors extended to the space of matrices. ${ }^{38}$

We compute the Frobenius distance between the two model-generated variance-covariance matrices, $\Sigma_{I O}^{m}$ and $\Sigma_{h o r}^{m}$, and the true one, $\Sigma_{\text {data }}^{m}$. Because both model-generated matrices have been normalized to have the same average variance as the true one, their relative "distances" to $\Sigma_{\text {data }}^{m}$ should indicate which of the two better matches the off-diagonal covariance elements. We obtain the following:

$$
\left\|\Sigma_{\text {data }}^{m}-\Sigma_{\text {hor }}^{m}\right\|=.74, \text { and }\left\|\Sigma_{\text {data }}^{m}-\Sigma_{I O}^{m}\right\|=.69
$$

The Frobenius distance between the variance-covariance sales matrix in the horizontal economy and that in the data is .74, while the Frobenius distance between the variance-covariance sales matrix in the calibrated network economy and that in the data is .69. We conclude that the calibrated network economy does a better job than the horizontal economy in matching the offdiagonal covariance elements of log sales found in the data.

Note that the ability of the model to match the sectoral covariances observed in the data is not just a function of the input-output network, but also of the GZ shocks themselves. However, the GZ distortions are only one particular measure of one particular type of shock. To the extent that there were other aggregate and sectoral shocks-productivities, non-financial distortions, etc.- hitting the economy during this period, the calibrated model with GZ distortions alone would not be able to account for all of the covariation observed in the data. Our next and final exercise takes this limitation into consideration.

\subsubsection{The Distribution of Distances.}

We have hereby shown that $\Sigma_{\text {data }}^{m}$ is closer in Frobenius distance to $\Sigma_{I O}^{m}$ than it is to $\Sigma_{h o r}^{m}$. But how much closer? What is a reasonable distance given only the GZ shocks?

In our final exercise we aim to gain a better understanding of what is relatively "close" and what is relatively "far" conditional on the GZ distortion series. To do so, we run a Monte Carlo experiment in which we simulate the economy 6000 times, each time with a different input-output matrix. Specifically, for each simulation we generate a random input-output matrix. ${ }^{39}$ In each economy we feed in the same GZ distortion series, compute the model-generated series of log

\footnotetext{
${ }^{38}$ The Frobenius norm is also equal to the square root of the matrix trace of $(A-B)^{\prime}(A-B)$.

${ }^{39}$ For each simulation we use the same consumption share vector and the same labor share vector. It is only the input-output network that is randomized.
} 
sectoral sales, and then compute its normalized variance-covariance matrix. Finally, we measure the Frobenius distance between the simulated variance-covariance matrix and that measured in the data. We plot the histogram of these distances generated by the 6000 simulations in Figure 10.

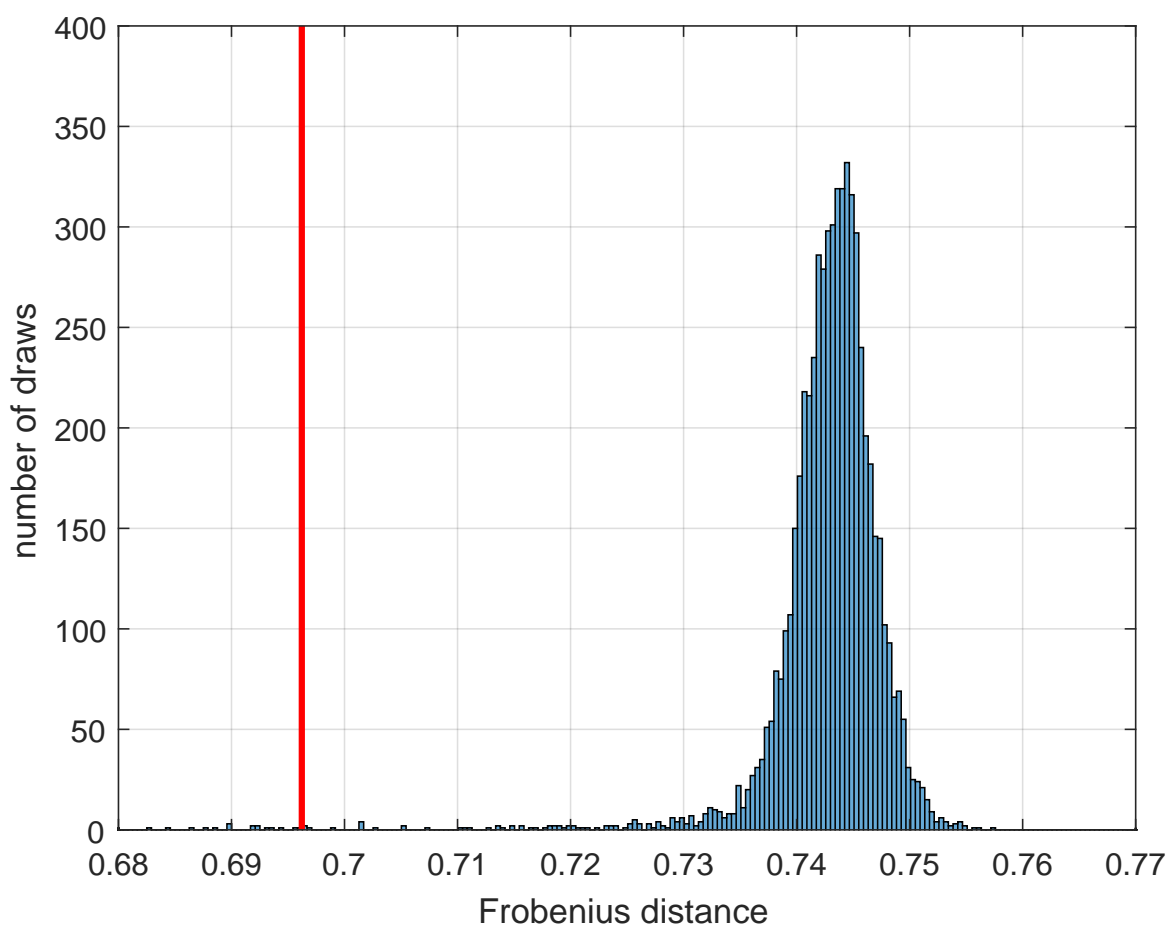

Figure 10: Histogram of Frobenius distances from Monte Carlo simulations

The histogram is a simulated probability density of the distances that could be generated by the underlying model given the series of underlying GZ shocks. We indicate where the calibrated network economy falls in this distribution by the red vertical line; it is clearly to left of the majority of the distribution's mass. To be precise, we find that in fact only $0.51 \%$ of these distances are less than .69. We conclude that the model calibrated to the U.S input-output network does relatively well in matching the sectoral covariances present in the sectoral sales data.

\subsection{Summary of findings and relation to subsequent work}

In this quantitative illustration, we calibrate the model to the US input-output tables and use the Gilchrist and Zakrajšek (2012) excess bond premia as our measure of sectoral financial frictions. This exercise produces three main results:

1. The model generates quantitatively larger movements in the aggregate labor wedge than in TFP over our sample period. Movements in the model-implied aggregate labor wedge are relatively correlated with its counterpart in the data. 
2. The model produces a labor wedge network multiplier of roughly 2 , indicating that the U.S. input-output network may have generated considerable amplification of financial shocks during the crisis.

3. The calibrated network economy better matches the cross-sectional covariance in sales than its equivalent horizontal economy; in fact it outperforms $99.5 \%$ of random input-output networks.

In this application we interpret the distortions as financial frictions generated by working capital constraints. A clear limitation of our approach is that we treat firms' financial constraints as exogenous: we do not explicitly model how firms obtain credit. Three recent papers by Altinoglu (2018), Reischer (2019), and Luo (2016) extend our framework by endogenizing this friction through trade credit. These papers study input-output economies in which firms must finance working capital partly through trade credit. Trade credit is provided by intermediate good suppliers and, as a result, input-output linkages coincide with financial linkages.

Altinoglu (2018) is the first to explicitly model trade credit in an input-output economy. In his model, working capital is funded by both bank and trade credit, the latter is received from suppliers and extended to customers. Altinoglu (2018) shows how fixed-proportion pledgeability constraints on these channels reduce to the working capital constraints we consider; the tightness of these constraints, however, are endogenous and depend on the cash-flow of downstream firms. This is a new mechanism Altinoglu (2018) calls the "credit linkage channel" and he shows how it may further amplify financial shocks.

Reischer (2019) further endogenizes trade credit in an input-output economy by allowing firms to choose both the composition of their borrowing and the trade credit they extend to customers. In her model, firms face a trade-off between the interest rates and management costs of different credit channels; there are in turn functions of trade credit extended to customers. Reischer (2019) shows how these frictions reduce to the sectoral wedges we consider. However, the wedges in her model are endogenous - they are weighted averages of the interest rates on bank and supplier credit where the weights are the firm's optimally chosen link-specific trade-credit shares.

Finally in Luo (2016), trade credit directly affects input prices. Suppliers value early payments and charge customer-specific prices: the greater the trade credit they must extend, the greater the price they charge. Firms take this into account when choosing their input-specific credit mix and choose a borrowing composition that minimizes their total costs of production.

Our model is nested in all three papers as the reduced-form version in which working capital constraints are exogenous. 


\section{Conclusion}

This paper studies the macroeconomic effects of sectoral distortions in an input-output production network. Our theoretical analysis provides three main results.

1. Rebated distortions manifest at the aggregate level through two channels: the labor wedge and a fall in TFP. Sectoral productivity shocks and wasted distortions result in TFP movement alone.

2. Small rebated distortions have zero first-order effects on TFP but non-zero first-order effects on the aggregate labor wedge. The magnitude of the latter coincide with sectors' "centrality."

3. Sufficient statistics for the first-order effects of small rebated distortions are the Domar weights.

We use three examples to illustrate how different network structures lead to different aggregate effects of distortions. Finally, we apply the model to the 2008 Financial Crisis and consider the effects of financial shocks, as measured by the Gilchrist and Zakrajšek (2012) excess bond premia. In particular, we find that the U.S. input-ouput network amplifies financial distortions by roughly a factor of two relative to an economy without intermediate good trade.

There are obvious limitations to our analysis. One is the restriction to Cobb-Douglas technologies thereby imposing an elasticity of substitution of 1 across all inputs. Another clear restriction is perfect mobility across labor markets. An elasticity of substitution across lower than one - as is estimated in Atalay (2015) - and a less than perfectly mobile labor market would make reallocation of inputs across sectors more sclerotic. As a result, we conjecture that sectoral distortions would have an even more pronounced aggregate effect. For results in input-output economies with more general production functions, see the work of Atalay (2015); Baqaee and Farhi (2019a,b).

Another limitation is the exogeneity of distortions, in particular the financial frictions highlighted in the quantitative exercise. As we have noted, recent papers by Altinoglu (2018), Reischer (2019) and Luo (2016) present innovative ways to tackle this limitation in the context of financial shocks and trade credit linkages. Moreover, recent empirical work by Costello (2018) provides evidence that suppliers exposed to large exogenous declines in bank financing pass their shock to their downstream customers through a reduction in the trade credit.

To conclude, despite the limitations of the model and the quantitative exercise, we hope that this paper offers new insight on the network effects of sectoral distortions and motivates future research in this field. 


\section{References}

Acemoglu, Daron, Vasco M. Carvalho, Asuman Ozdaglar, and Alireza Tahbaz-Salehi, "The Network Origins of Aggregate Fluctuations," Econometrica, 2012, 80 (5), 1977-2016. 1, 1, $1,4.1,12,4.1,13$

Altinoglu, Engin L., "The Origins of Aggregate Fluctuations in a Credit Network Economy," Finance and Economics Discussion Series 2018-031, Board of Governors of the Federal Reserve System (US) 2018. 1, 3, 6.5, 7

Antràs, Pol and Alonso de Gortari, "On the Geography of Global Value Chains," 2017. 5

_, Teresa C. Fort, and Felix Tintelnot, "The Margins of Global Sourcing: Theory and Evidence from U.S. Firms," American Economic Review, 2017 2017, 107 (9), 2514-64. 5

Asker, John, Allan Collard-Wexler, and Jan De Loecker, "Dynamic Inputs and Resource (Mis)Allocation," Journal of Political Economy, 2014, 122 (5), 1013-1063. 1

Atalay, Enghin, "How Important Are Sectoral Shocks," 2015. UW Madison Working Paper. 7

Baldwin, Richard and Anthony J. Venables, "Spiders and snakes: Offshoring and agglomeration in the global economy," Journal of International Economics, 2013, 90 (2), 245 - 254. 5

Baqaee, David and Emmanuel Farhi, "Productivity and Misallocation in General Equilibrium," 2019. 1, 2, 3, 9, 4.4, 19, 7

Baqaee, David Rezza and Emmanuel Farhi, "The Macroeconomic Impact of Microeconomic Shocks: Beyond Hulten's Theorem," Econometrica, 2019, 87 (4), 1155-1203. 3, 7

Basu, Susanto, "Intermediate Goods and Business Cycles: Implications for Productivity and Welfare," American Economic Review, June 1995, 85 (3), 512-31. 1, 1, 7

Bernanke, Ben and Mark Gertler, "Agency Costs, Net Worth, and Business Fluctuations," American Economic Review, March 1989, 79 (1), 14-31. 7

Bigio, Saki, "Endogenous Liquidity and the Business Cycle," American Economic Review, 2015, 105 (6), 1883-1927. 7

Burress, David, "Homeomorphism between Leontief and Cobb-Douglas input-output models," Economics Letters, 1994, 44 (1-2), 49-53. 1, 4.1, 13 
Carvalho, Vasco M., "From Micro to Macro via Production Networks," The Journal of Economic Perspectives, 2014, 28 (4), 23-47. 1, 4.1

Chari, V. V., Patrick J. Kehoe, and Ellen R. McGrattan, "Business Cycle Accounting," Econometrica, 2007, 75 (3), 781-836. 1, 4, 14

Ciccone, Antonio, "Input Chains and Industrialization," The Review of Economic Studies, 2002, $69(3), 565-587.1,1$

Costello, Anna, "Credit Market Disruptions and Liquidity Spillover Effects in the Supply Chain," SSRN Electronic Journal, 01 2018. 7

Diamond, Peter A. and James A. Mirrlees, "Optimal Taxation and Public Production I: Production Efficiency," The American Economic Review, 1971, 61 (1), 8-27. 4.4, 5.1, 5.3

Dupor, Bill, "Aggregation and irrelevance in multi-sector models," Journal of Monetary Economics, 1999, 43 (2), $391-409.1$

Fadinger, Harald, Christian Ghiglino, and Mariya Teteryatnikova, "Income Differences, Productivity and Input-Output Networks," 2018. 4

Gabaix, Xavier, "The Granular Origins of Aggregate Fluctuations," Econometrica, 05 2011, 79 (3), 733-772. 1, 1, 13

Gertler, Mark and Peter Karadi, "A model of unconventional monetary policy," Journal of Monetary Economics, 2011, 58 (1), 17-34. 7

Gilchrist, Simon and Egon Zakrajšek, "Credit Spreads and Business Cycle Fluctuations," American Economic Review, 2012, 102 (4), 1692-1720. 1, 6, 6.1, 6.3, 6.5, 7, 7

Greenwood, Jeremy, Zvi Hercowitz, and Gregory W Huffman, "Investment, Capacity Utilization, and the Real Business Cycle," American Economic Review, June 1988, 78 (3), 402417. 31,32

Hall, Robert E., "Macroeconomic Fluctuations and the Allocation of Time," Journal of Labor Economics, 1997, 15 (1), pp. S223-S250. 14

_, "Reconciling Cyclical Movements in the Marginal Value of Time and the Marginal Product of Labor," Journal of Political Economy, 04 2009, 117 (2), 281-323. 32

Horvath, Michael, "Cyclicality and Sectoral Linkages: Aggregate Fluctuations from Independent Sectoral Shocks," Review of Economic Dynamics, 1998, 1 (4), 781 - 808. 1 
_ , "Sectoral shocks and aggregate fluctuations," Journal of Monetary Economics, 2000, 45 (1), 69 - 106. 1

Hsieh, Chang-Tai and Peter J. Klenow, "Misallocation and Manufacturing TFP in China and India," The Quarterly Journal of Economics, November 2009, 124 (4), 1403-1448. 5.1

Hulten, Charles R., "Growth Accounting with Intermediate Inputs," The Review of Economic Studies, 1978, 45 (3), pp. 511-518. 1, 1, 3, 4.4, 4.4, 7

Jermann, Urban and Vincenzo Quadrini, "Macroeconomic Effects of Financial Shocks," 2011. American Economic Review, forthcoming. 7

Jones, Charles I., "Intermediate Goods and Weak Links in the Theory of Economic Development," American Economic Journal: Macroeconomics, 2011, 3 (2), pp. 1-28. 1, 1, 6.3.2, 34, 7, $7,7,7$

_ , "Misallocation, Economic Growth, and Input-Output Economies," in "Advances in Economics and Econometrics, Tenth World Congress, Volume II," Cambridge University Press, 2013. 1, 2, $4,4,10,20,26,6.3 .2,34,7,7,7,7$

Kalemli-Ozcan, Sebnem, Hyun Song Shin, Se-Jik Kim, Bent Sorensen, and Sevcan Yesiltas, "Financial Shocks in Production Chains," 2014. 22

Kiyotaki, Nobuhiro and John Moore, "Credit Cycles," Journal of Political Economy, April 1997, $105(2), 211-48.7$

Liu, Ernest, "Industrial Policies in Production Networks," 2017. Unpublished Manuscript. 1

Long, John B. Jr and Charles I Plosser, "Real Business Cycles," Journal of Political Economy, February 1983, 91 (1), 39-69. 1, 1, 1

Luo, Shaowen, "Propagation of Financial Shocks in an Input-Output Economy with Trade and Financial Linkages of Firms," 2016. Columbia University Job Market Paper. 1, 3, 6.5, 7

Merton, Robert C., "On the pricing of corporate debt: the risk structure of interest rates," The Journal of Finance, 1974, 29 (2), 449-470. 6.1

Reischer, Margit, "Finance-Thy-Neighbor: Trade Credit Origins of Aggregate Fluctuations.," 2019. University of Cambridge Job Market Paper. 1, 3, 6.5, 7

Restuccia, Diego and Richard Rogerson, "Policy Distortions and Aggregate Productivity with Heterogeneous Plants," Review of Economic Dynamics, October 2008, 11 (4), 707-720. 1, 5.1 
Rotemberg, Julio J. and Michael Woodford, "The cyclical behavior of prices and costs," in J. B. Taylor and M. Woodford, eds., Handbook of Macroeconomics, Vol. 1 of Handbook of Macroeconomics, Elsevier, 1999, chapter 16, pp. 1051-1135. 14

Yi, Kei-Mu, "Can Vertical Specialization Explain the Growth of World Trade?," Journal of Political Economy, 2003, 111 (1), 52-102. 1, 1 


\section{Appendix A: Proofs}

In this Appendix, we first state and solve the household and firms' problems. We then prove two auxiliary lemmas which do not appear in the main text but offer a complete characterization of general equilibrium allocations in our economy. We then proceed with the proofs of all results that appear in the main text. Finally we end Appendix A with a lemma that provides closed-form solutions for aggregate consumption and labor under homothetic preferences as well as its proof.

\section{Auxiliary Problems, Lemmas, and Proofs}

In this auxiliary section of the Appendix we do not yet impose the Cobb-Douglas assumption on technology and preferences. That is, we let the production function of firm $k$ in sector $i$ be given by

$$
y_{i, k}=z_{i} F_{i}\left(\ell_{i, k}, x_{i, k}\right)
$$

where $F_{i}$ is homothetic of degree 1 . The composite of intermediate goods is given by

$$
x_{i, k} \equiv G_{i}\left(\mathbf{x}_{i, k}\right)
$$

where $G_{i}$ is homothetic of degree 1. Finally, in terms of household preferences over goods, we let

$$
C=\mathcal{C}\left(c_{1}, \ldots, c_{N}\right)
$$

where $\mathcal{C}$ is homogenous of degree 1.

Consider first the household. The household solves the following problem.

The Household's Problem. In any state s, the household chooses its consumption bundle and labor supply $\left\{c_{1}(s), \ldots, c_{N}(s), L(s)\right\}$ in order to maximize its utility

$$
\max U\left(\mathcal{C}\left(c_{1}(s), \ldots, c_{N}(s)\right)\right)-V(L(s))
$$

subject to its budget constraint

$$
\sum_{i \in I} p_{i}(s) c_{i}(s) \leq L(s)+\sum_{i \in I}\left(\int \pi_{i k}(s) d k+\bar{\pi}_{i}(s)\right)+T(s)
$$

taking the price vector $\mathbf{p}(s)=\left(p_{1}(s), \ldots, p_{N}(s)\right)$ as given .

Consider now the production side. The sectoral aggregator firm solves the following problem.

The Sectoral Aggregator's Problem. In any state s, the sectoral aggregator firm in sector $i$ 
chooses output and input bundle, $\left\{y_{i}(s),\left(y_{i, k}(s)\right)_{k}\right\}$, in order to maximize profit

$$
\max p_{i}(s) y_{i}(s)-\int p_{i, k}(s) y_{i, k}(s) d k
$$

subject to its technology

$$
y_{i}(s)=\left[\int y_{i, k}(s)^{\frac{\theta_{i}-1}{\theta_{i}}} d k\right]^{\frac{\theta_{i}}{\theta_{i}-1}}
$$

taking prices $\left\{p_{i}(s),\left(p_{i, k}(s)\right)_{k}\right\}$ as given.

The sectoral aggregator firm's problem is standard; it yields the typical CES demand function

$$
y_{i, k}(s)=\left(\frac{p_{i, k}(s)}{p_{i}(s)}\right)^{-\theta_{i}} y_{i}(s)
$$

where the price index for sector $i$ is given by

$$
p_{i}(s)=\left(\int p_{i, k}(s)^{1-\theta_{i}} d k\right)^{\frac{1}{1-\theta_{i}}}
$$

Finally consider the problem of the typical monopolistically-competitive firm within a production sector.

The Monopolist's Problem. In any state $s$, the monopolistically-competitive firm $k$ within sector $i$ chooses output and inputs $\left(y_{i, k}(s), \ell_{i, k}(s), \mathbf{x}_{i, k}(s)\right)$ so as to maximize its profit

$$
\max \left(1-\tau_{i}(s)\right) p_{i, k}(s) y_{i, k}(s)-\ell_{i, k}(s)-\sum_{j \in I} p_{j}(s) x_{i j, k}(s)
$$

subject to its technology,

$$
\begin{aligned}
y_{i, k}(s) & =z_{i}(s) F_{i}\left(\ell_{i, k}(s), x_{i, k}(s)\right) \\
x_{i, k}(s) & =G_{i}\left(\mathbf{x}_{i, k}(s)\right)
\end{aligned}
$$

and subject to the demand for its product,

$$
y_{i, k}(s)=\left(\frac{p_{i, k}(s)}{p_{i}(s)}\right)^{-\theta_{i}} y_{i}(s) .
$$

taking input prices as given. 
Finally, market clearing for good $i$ in state $s$ satisfies

$$
y_{i}(s)=c_{i}(s)+h_{i}(s)+\sum_{j \in I} x_{j i}(s), \text { with } y_{i}(s)=z_{i}(s) F_{i}\left(\ell_{i}(s), x_{i}(s)\right) \quad \forall i \in I
$$

the market for labor clears if and only if

$$
\sum_{i \in I} \ell(s)=L(s) \quad \forall s \in S
$$

Equilibrium characterization Lemma and Proposition. We now state an auxiliary lemma and proposition, followed by their proofs. The following lemma provides a complete characterization of the equilibrium.

Lemma 2. An allocation $\xi$ and a price system @ compose an equilibrium if and only if the following four properties hold.

(i) The following household optimality conditions are satisfied:

$$
\begin{aligned}
U^{\prime}(C(s)) \frac{d \mathcal{C}(s)}{d c_{i}(s)} \frac{1}{p_{i}(s)} & =V^{\prime}(L(s)), \quad \forall i \in I, s \in S \\
\frac{d \mathcal{C}(s) / d c_{j}(s)}{d \mathcal{C}(s) / d c_{i}(s)} & =\frac{p_{j}(s)}{p_{i}(s)}, \quad \forall i, j \in I, s \in S
\end{aligned}
$$

(ii) The following firm optimality conditions are satisfied:

$$
\begin{aligned}
\left(\frac{\theta_{i}(s)-1}{\theta_{i}(s)}\right)\left(1-\tau_{i}(s)\right)\left(z_{i}(s) \frac{d F_{i}(s)}{d \ell_{i}(s)}\right)-\frac{1}{p_{i}(s)} & =0, \quad \forall i \in I, s \in S \\
\left(\frac{\theta_{i}(s)-1}{\theta_{i}(s)}\right)\left(1-\tau_{i}(s)\right)\left(z_{i}(s) \frac{d F_{i}(s)}{d x_{i}(s)} \frac{d G_{i}(s)}{d x_{i j}(s)}\right)-\frac{p_{j}(s)}{p_{i}(s)} & =0, \quad \forall i, j \in I, s \in S
\end{aligned}
$$

along with the intermediate-good CES demand condition:

$$
y_{i, k}(s)=\left(\frac{p_{i, k}(s)}{p_{i}(s)}\right)^{-\theta_{i}} y_{i}(s), \quad \forall s \in S
$$

(iii) The household's budget constraint is satisfied.

(iv) All markets clear, namely, conditions (47) and (48), are satisfied.

Lemma 3. An allocation $\xi$ is part of an equilibrium if and only if it satisfies the following set of conditions 


$$
\begin{aligned}
V^{\prime}(L(s)) & =\left(\frac{\theta_{i}(s)-1}{\theta_{i}(s)}\right)\left(1-\tau_{i}(s)\right) U^{\prime}(C(s)) \frac{d \mathcal{C}(s)}{d c_{i}(s)} z_{i}(s) \frac{d F_{i}(s)}{d \ell_{i}(s)}, \forall i \in I, s \in S \\
\frac{d \mathcal{C}(s) / d c_{j}(s)}{d \mathcal{C}(s) / d c_{i}(s)} & =\left(\frac{\theta_{i}(s)-1}{\theta_{i}(s)}\right)\left(1-\tau_{i}(s)\right) z_{i}(s) \frac{d F_{i}(s)}{d x_{i}(s)} \frac{d G_{i}(s)}{d x_{i j}(s)} \quad \forall i, j \in I, s \in S
\end{aligned}
$$

and resource constraints

$$
L(s)=\sum_{i \in I} \ell_{i}(s) \text { and } y_{i}(s)=c_{i}(s)+h_{i}(s)+\sum_{j \in I} x_{j i}(s), \quad \forall i \in I, s \in S
$$

where $h_{i}(s) \equiv \delta_{i}(s) \tau_{i}(s) y_{i}(s)$

Proof of Lemma 2. We first derive the household's optimality conditions. Following this we derive the firm's optimality conditions.

Household. Consider the household's problem stated above. Let $\lambda(s)$ be the Lagrange multiplier on the Household's budget constraint for state $s$. The household's first order conditions for consumption of any good and labor are given by

$$
\begin{aligned}
U^{\prime}(C(s)) \frac{d \mathcal{C}(s)}{d c_{i}(s)}-\lambda(s) p_{i}(s) & =0 \quad \forall i \in I, s \in S \\
-V^{\prime}(L(s))+\lambda(s) & =0 \quad \forall s \in S
\end{aligned}
$$

By combining (57) and (58) we derive the household's optimality condition stated in (51). By combining (57) for good $i$ and (57) for good $j$ we derive the household's optimality condition stated in $(52)$.

The Sectoral Aggregator Firm. The sectoral aggregator firm's problem is straight-forward and results in the CES demand function given in (53).

The Monopolistically-Competitive Firm. Next consider the typical monopolistically-competitive firm within sector $i$. The firm's problem can be split into a dual problem. The firm solves an outer problem of profit maximization given by

$$
\max \left(1-\tau_{i}(s)\right) p_{i, k}(s) y_{i, k}(s)-m c_{i, k}(s) y_{i, k}(s)
$$

subject to the firm's demand function (53).

$$
y_{i, k}(s)=\left(\frac{p_{i, k}(s)}{p_{i}(s)}\right)^{-\theta_{i}} y_{i}(s)
$$


where $m c_{i, k}(s)$ is the firm's marginal cost of producing goods $y_{i, k}(s)$. Substituting in the firm's demand function, this problem reduces to

$$
\max \left(1-\tau_{i}(s)\right)\left(\frac{y_{i, k}(s)}{y_{i}(s)}\right)^{-1 / \theta_{i}} p_{i}(s) y_{i, k}(s)-m c_{i, k}(s) y_{i, k}(s)
$$

which yields optimality condition

$$
\left(\frac{\theta_{i}(s)-1}{\theta_{i}(s)}\right)\left(1-\tau_{i}(s)\right)\left(\frac{y_{i, k}(s)}{y_{i}(s)}\right)^{-1 / \theta_{i}} p_{i}(s)=m c_{i, k}(s)
$$

By symmetry of all firms within the sector, we get

$$
\left(\frac{\theta_{i}(s)-1}{\theta_{i}(s)}\right)\left(1-\tau_{i}(s)\right) p_{i}(s)=m c_{i}(s)
$$

The inner problem determines the firm's marginal cost function. In particular, the firm's inner problem is a cost minimization problem given by

$$
m c_{i}(s) y_{i}(s)=\min \ell_{i}(s)+\mathbf{p}(s)^{\prime} \mathbf{x}_{i}(s)
$$

where we let $\mathbf{p}(s)=\left(p_{1}(s), \ldots, p_{N}(s)\right)^{\prime}$ denote the vector of commodity prices, subject to the firm's production function

$$
y_{i}(s)=z_{i}(s) F_{i}\left(\ell_{i}(s), G_{i}\left(\mathbf{x}_{i}(s)\right)\right)
$$

This yields optimality conditions

$$
m c_{i}(s)=\left(z_{i}(s) \frac{d F_{i}(s)}{d \ell_{i}(s)}\right)^{-1} \text { and } m c_{i}(s)=p_{j}(s)\left(z_{i}(s) \frac{d F_{i}(s)}{d x_{i}(s)} \frac{d G_{i}(s)}{d x_{i j}(s)}\right)^{-1}
$$

From now on we drop the subscript $k$ on the firm's marginal cost. Combining the marginal cost conditions in (63) with (61) we obtain optimality conditions for the firm stated in (51) and (52). The firm and household's optimality conditions, along with budget constraints and market clearing conditions, constitute an equilibrium. QED.

Proof of Lemma 3. Necessity. We first prove necessity. First, take equation (51). This may be rewritten as

$$
\left(\frac{\theta_{i}-1}{\theta_{i}}\right)\left(1-\tau_{i}\right)\left(z_{i}(s) \frac{d F_{i}(s)}{d \ell_{i}(s)}\right)=\frac{1}{p_{i}(s)} \quad \forall i \in I, s \in S
$$


Combining this with the household's intratemporal condition between good $i$ and labor, (49), we obtain

$$
\left(\frac{\theta_{i}-1}{\theta_{i}}\right)\left(1-\tau_{i}\right)\left(z_{i}(s) \frac{d F_{i}(s)}{d \ell_{i}(s)}\right) U^{\prime}(C(s)) \frac{d \mathcal{C}(s)}{d c_{i}(s)}=V^{\prime}(L(s)) \quad \forall i \in I, s \in S
$$

thereby proving necessity of (54).

Next, take equation (52). This may be rewritten as

$$
\left(\frac{\theta_{i}-1}{\theta_{i}}\right)\left(1-\tau_{i}\right)\left(z_{i}(s) \frac{d F_{i}(s)}{d x_{i}(s)} \frac{d G_{i}(s)}{d x_{i j}(s)}\right)=\frac{p_{j}(s)}{p_{i}(s)} \quad \forall i, j \in I, s \in S
$$

Combining this with the household's intratemporal condition between good $i$ and good $j$, (50), we obtain

$$
\left(\frac{\theta_{i}-1}{\theta_{i}}\right)\left(1-\tau_{i}\right)\left(z_{i}(s) \frac{d F_{i}(s)}{d x_{i}(s)} \frac{d G_{i}(s)}{d x_{i j}(s)}\right)=\frac{d \mathcal{C}(s) / d c_{j}(s)}{d \mathcal{C}(s) / d c_{i}(s)} \quad \forall i, j \in I, s \in S
$$

thereby proving necessity of (55).

Thus conditions (54) and (55) along with the resource constraints stated in (56) are necessary conditions on the equilibrium allocation.

Sufficiency. Consider now sufficiency. Take any allocation $\xi(s)$ that satisfies conditions (54)(55) along with resource constraints (56). We now prove that there exists a set of prices $\mathbf{p}(s)=$ $\left(p_{1}(s), \ldots, p_{N}(s)\right)$ that implement this allocation as an equilibrium. We construct the equilibrium prices as follows.

We start off by setting the wage equal to 1 (as in our equilibrium definition). We then set each sectoral price as follows

$$
p_{i}(s)=\frac{U^{\prime}(C(s))}{V^{\prime}(L(s))} \frac{d \mathcal{C}(s)}{d c_{i}(s)}
$$

so as to satisfy household optimality condition (49). Prices given in (64) along with the fact that the allocation satisfies (54) ensures that firm optimality (51) is satisfied.

Next, it is clear that if you take the ratio of prices $p_{j}(s) / p_{i}(s)$ given in (64), we have that household optimality condition (50) is satisfied:

$$
\frac{p_{j}(s)}{p_{i}(s)}=\frac{d \mathcal{C}(s) / d c_{j}(s)}{d \mathcal{C}(s) / d c_{i}(s)} \quad \forall i, j \in I, s \in S
$$

The price ratio in (65) along with the fact that the allocation satisfies (55) ensures that firm optimality (52) is satisfied.

Finally, for any sector $i$, let relative firm prices satisfy

$$
\frac{p_{i, k}(s)}{p_{i}(s)}=\left(\frac{y_{i, k}(s)}{y_{i}(s)}\right)^{-\frac{1}{\theta_{i}}} \quad \forall s \in S
$$


With these prices we satisfy the equilibrium conditions (53) for intermediate good demand. Given that $y_{i, k}(s)=y_{i}(s)$ for all firms $k$ in sector $i$, this immediately implies that $p_{i, k}(s)=p_{i}(s)$ for all $k$.

Market clearing for each good is ensured by the resource constraints in (56). Finally, what remains to be checked is that the household's budget constraint is satisfied. Consider the household budget constraint

$$
\sum_{i \in I} p_{i}(s) c_{i}(s) \leq L(s)+\sum_{i \in I}\left(\int \pi_{i k}(s) d k+\pi_{i}(s)\right)+T(s)
$$

Substituting in for profits from (45) we have that the household's budget constraint satisfies

$$
\sum_{i \in I} p_{i}(s) c_{i}(s) \leq L(s)+\sum_{i \in I}\left(\left(1-\tau_{i}(s)\right) p_{i}(s) y_{i}(s)-\ell_{i}(s)-\sum_{j \in I} p_{j}(s) x_{i j}(s)\right)+T(s) .
$$

Using the resource constraint for labor (56), this inequality reduces to

$$
\sum_{i \in I} p_{i}(s) c_{i}(s) \leq \sum_{i \in I}\left(1-\tau_{i}(s)\right) p_{i}(s) y_{i}(s)-\sum_{i \in I} \sum_{j \in I} p_{j}(s) x_{i j}(s)+T(s) .
$$

Next, take the resource constraint (56) for good $i$ and multiply both sides by $p_{i}(s)$; this yields

$$
p_{i}(s) y_{i}(s)=p_{i}(s) c_{i}(s)+p_{i}(s) h_{i}(s)+\sum_{j \in I} p_{i}(s) x_{j i}(s)
$$

Summing this expression across sectors and rearranging gives us

$$
\sum_{i \in I} p_{i}(s) c_{i}(s)=\sum_{i \in I} p_{i}(s) y_{i}(s)-\sum_{i \in I} p_{i}(s) h_{i}(s)-\sum_{i \in I} \sum_{j \in I} p_{i}(s) x_{j i}(s)
$$

Combining this expression with condition (66) gives us

$$
\sum_{i \in I} p_{i}(s) y_{i}(s)-\sum_{i \in I} p_{i}(s) h_{i}(s)-\sum_{i \in I} \sum_{j \in I} p_{i}(s) x_{j i}(s) \leq \sum_{i \in I}\left(1-\tau_{i}(s)\right) p_{i}(s) y_{i}(s)-\sum_{i \in I} \sum_{j \in I} p_{j} x_{i j}(s)+T(s) .
$$

Canceling terms on both sides and rearranging yields

$$
\sum_{i \in I} p_{i}(s) y_{i}(s) \leq \sum_{i \in I}\left(1-\tau_{i}(s)\right) p_{i}(s) y_{i}(s)+\sum_{i \in I} p_{i}(s) h_{i}(s)+T(s)
$$


Using the fact that tax proceeds wasted and rebated satisfy

$$
\sum_{i \in I} p_{i}(s) h_{i}(s)=\sum_{i \in I} \delta_{i}(s) \tau_{i}(s) p_{i}(s) y_{i}(s) \text { and } T(s)=\sum_{i \in I}\left(1-\delta_{i}(s)\right) \tau_{i}(s) p_{i}(s) y_{i}(s)
$$

we may re-express equation (66) as follows

$\sum_{i \in I} p_{i}(s) y_{i}(s) \leq \sum_{i \in I}\left(1-\tau_{i}(s)\right) p_{i}(s) y_{i}(s)+\sum_{i \in I} \delta_{i}(s) \tau_{i}(s) p_{i}(s) y_{i}(s)+\sum_{i \in I}\left(1-\delta_{i}(s)\right) \tau_{i}(s) p_{i}(s) y_{i}(s)$.

Collecting terms we may be rewrite this as

$$
\sum_{i \in I} p_{i}(s) y_{i}(s) \leq \sum_{i \in I}\left[1-\tau_{i}(s)+\delta_{i}(s) \tau_{i}(s)+\left(1-\delta_{i}(s)\right) \tau_{i}(s)\right] p_{i}(s) y_{i}(s)
$$

which holds with equality. Therefore, the resource constraints in (56) ensure that the household budget constraint is satisfied. QED.

\section{Proofs for the Main Text}

We now provide the proofs for all lemmas, propositions, and theorems that appear in the main text.

Proof of Proposition 1. First, consider the resource constraint for sector $i$ given in (56). Using the fact that $h_{i}(s)=\delta_{i}(s) \tau_{i}(s) y_{i}(s)$, we may rewrite this resource constraint as

$$
y_{i}(s)-\delta_{i}(s) \tau_{i}(s) y_{i}(s)=c_{i}(s)+\sum_{j \in I} x_{j i}(s)
$$

Next, we define a function $q_{i}(s)$ as follows

$$
q_{i}(s) \equiv y_{i}(s)-h_{i}(s)=\left(1-\delta_{i}(s) \tau_{i}(s)\right) y_{i}(s)
$$

Substituting this into (68) gives us

$$
q_{i}(s)=c_{i}(s)+\sum_{j \in I} x_{j i}(s)
$$

This verifies that the resource constraints in (10) preserves the resource constraints in (56) with the functions $q_{i}(s)$ and $\psi(s)$ as defined in (7) and (6), respectively.

Next, we rewrite the conditions (54) and (55) in Proposition 3 in terms of the Cobb-Douglas 
technologies and preferences. This yields the following conditions:

$$
\begin{aligned}
V^{\prime}(L(s)) & =\left(\frac{\theta_{i}(s)-1}{\theta_{i}(s)}\right)\left(1-\tau_{i}(s)\right) U^{\prime}(C(s)) v_{i} \frac{C(s)}{c_{i}(s)} \alpha_{i} \frac{y_{i}(s)}{\ell_{i}(s)} \quad \forall i, s, \\
\frac{v_{j} C(s) / c_{j}(s)}{v_{i} C(s) / c_{i}(s)} & =\left(\frac{\theta_{i}(s)-1}{\theta_{i}(s)}\right)\left(1-\tau_{i}(s)\right)\left(1-\alpha_{i}\right) g_{i j} \frac{y_{i}(s)}{x_{i j}(s)} \quad \forall i, j, s .
\end{aligned}
$$

Multiplying and dividing the right-hand side of these equations by $q_{i}(s)$ gives us the following equilibrium conditions:

$$
\begin{aligned}
V^{\prime}(L(s)) & =\left(\frac{\theta_{i}(s)-1}{\theta_{i}(s)}\right)\left(\frac{1-\tau_{i}(s)}{1-\delta_{i}(s) \tau_{i}(s)}\right) U^{\prime}(C(s)) v_{i} \frac{C(s)}{c_{i}(s)} \alpha_{i} \frac{q_{i}(s)}{\ell_{i}(s)} \quad \forall i, s, \\
\frac{v_{j} C(s) / c_{j}(s)}{v_{i} C(s) / c_{i}(s)} & =\left(\frac{\theta_{i}(s)-1}{\theta_{i}(s)}\right)\left(\frac{1-\tau_{i}(s)}{1-\delta_{i}(s) \tau_{i}(s)}\right)\left(1-\alpha_{i}\right) g_{i j} \frac{q_{i}(s)}{x_{i j}(s)} \quad \forall i, j, s .
\end{aligned}
$$

This verifies that optimality conditions (8) and (9) are equivalent to optimality conditions (54) and (55) in Proposition 3 with the function $\phi(s)$ as defined in (6). QED.

Proof of Proposition 2. Relative to the baseline economy, the only modification is that firm profits are now given by

$$
\pi_{i, k}(s)=p_{i, k}(s) y_{i, k}(s)-\left(1+r_{i}(s)\right)\left(\ell_{i}(s)+\sum_{j \in I} p_{j}(s) x_{i j, k}(s)\right)
$$

where $1+r_{i}$ is the intra-period interest rate. The firm chooses inputs and output in order maximize profits subject to the firm's demand function (53).

Following the proof of Lemma 2, the firm's inner cost-minimization problem remains the same. The firm's outer problem becomes

$$
\max _{y_{i, k}} p_{i, k}(s) y_{i, k}(s)-\left(1+r_{i}(s)\right) m c_{i}(s) y_{i, k}(s)
$$

subject to CES demand function (53), where $m c_{i}(s)$ denotes the firm's marginal cost as characterized in (63). This problem yields the following firm optimality condition

$$
\left(\frac{\theta_{i}(s)-1}{\theta_{i}(s)}\right)\left(\frac{y_{i, k}(s)}{y_{i}(s)}\right)^{-1 / \theta_{i}} p_{i}(s)=\left(1+r_{i}(s)\right) m c_{i}(s) .
$$

Imposing symmetry of all firms within a sector and taking the limit as $\theta_{i}(s) \rightarrow 1$, this condition becomes

$$
\frac{1}{1+r_{i}(s)} p_{i}(s)=m c_{i}(s)
$$


This condition is the financial friction economy's counterpart of condition (61) in the baseline economy. Finally, there are no wasted resources as the household budget constraint includes interest payments accrued to the banking sector. Therefore all parts of the proofs for Propositions 3 and 1 remain unchanged, modulo the redefinitions of $\boldsymbol{\phi}$ and $\boldsymbol{\psi}$ in (11). QED.

Proof of Theorem 1. First, from the equilibrium optimality condition between goods $j$ and $i$, equation (9), we have

$$
\frac{v_{i} / c_{i}(s)}{v_{j} / c_{j}(s)}=\phi_{j}(s)\left(1-\alpha_{j}\right) g_{j i} \frac{q_{j}(s)}{x_{j i}(s)} \quad \forall j, i, s .
$$

Rearranging condition (70) gives us

$$
x_{j i}(s)=\phi_{j}(s)\left(1-\alpha_{j}\right) g_{j i} q_{j}(s) \frac{v_{j}}{v_{i}} \frac{c_{i}(s)}{c_{j}(s)} \quad \forall j, i, s .
$$

Summing this expression across all goods $j$, gives us

$$
\sum_{j \in I} x_{j i}(s)=c_{i}(s) v_{i}^{-1} \sum_{j \in I} \phi_{j}(s)\left(1-\alpha_{j}\right) g_{j i}(s) v_{j} c_{j}(s)^{-1} q_{j}(s) .
$$

Plugging (72) into the resource constraint (10) for sector $i$, we get

$$
q_{i}(s)=c_{i}(s)+c_{i}(s) v_{i}^{-1} \sum_{j \in I} \phi_{j}(s)\left(1-\alpha_{j}\right) g_{j i}(s) v_{j} c_{j}(s)^{-1} q_{j}(s) \quad \forall i, s .
$$

Stacking this into vector form gives us

$$
\mathbf{q}(s)=\mathbf{c}(s)+\operatorname{diag}(\mathbf{c}(s))[\operatorname{diag}(\mathbf{v})]^{-1}(\operatorname{diag}(\boldsymbol{\phi}(s) \circ(\mathbf{1}-\boldsymbol{\alpha})) \mathbf{G})^{\prime} \operatorname{diag}(\mathbf{v})[\operatorname{diag}(\mathbf{c}(s))]^{-1} \mathbf{q}(s) .
$$

Thus, output $\boldsymbol{q}(s)$ satisfies

$$
\mathbf{q}(s)=\left\{\mathbb{I}_{N}-\operatorname{diag}(\mathbf{c}(s))[\operatorname{diag}(\mathbf{v})]^{-1}(\operatorname{diag}(\boldsymbol{\phi}(s) \circ(\mathbf{1}-\boldsymbol{\alpha})) \mathbf{G})^{\prime} \operatorname{diag}(\mathbf{v})[\operatorname{diag}(\mathbf{c}(s))]^{-1}\right\}^{-1} \mathbf{c}(s),
$$

and rearranging yields,

$$
\mathbf{q}(s)=\operatorname{diag}(\mathbf{c}(s))[\operatorname{diag}(\mathbf{v})]^{-1}\left\{\mathbb{I}_{N}-(\operatorname{diag}(\boldsymbol{\phi}(s) \circ(\mathbf{1}-\boldsymbol{\alpha})) \mathbf{G})^{\prime}\right\}^{-1} \operatorname{diag}(\mathbf{v})[\operatorname{diag}(\mathbf{c}(s))]^{-1} \mathbf{c}(s)
$$

Therefore

$$
\operatorname{diag}(\mathbf{v})[\operatorname{diag}(\mathbf{c}(s))]^{-1} \mathbf{q}(s)=\left\{\mathbb{I}_{N}-(\operatorname{diag}(\phi(s) \circ(\mathbf{1}-\boldsymbol{\alpha})) \mathbf{G})^{\prime}\right\}^{-1} \mathbf{v}
$$

Next we use the equilibrium optimality condition between labor and goods given in (8). Rear- 
ranging this equation, we may write labor in sector $i$ as follows

$$
\ell_{i}(s)=\frac{U^{\prime}(C(s)) C(s)}{V^{\prime}(L(s))} \phi_{i}(s) v_{i} \alpha_{i} \frac{q_{i}(s)}{c_{i}(s)} \quad \forall i, s .
$$

Stacking this into vector form gives us

$$
\ell(s)=\frac{U^{\prime}(C(s)) C(s)}{V^{\prime}(L(s))} \operatorname{diag}(\boldsymbol{\alpha} \circ \boldsymbol{\phi}(s) \circ \mathbf{v})[\operatorname{diag}(\mathbf{c}(s))]^{-1} \mathbf{q}(s) .
$$

Aggregating (74) to satisfy the labor market clearing condition gives us

$$
L(s)=\mathbf{1}^{\prime} \boldsymbol{\ell}(s)=\frac{U^{\prime}(C(s)) C(s)}{V^{\prime}(L(s))} \boldsymbol{\alpha}^{\prime} \operatorname{diag}(\boldsymbol{\phi}(s)) \operatorname{diag}(\mathbf{v})[\operatorname{diag}(\mathbf{c}(s))]^{-1} \mathbf{q}(s) .
$$

Combining (75) with (73) yields the following expression:

$$
L(s)=\frac{U^{\prime}(C(s)) C(s)}{V^{\prime}(L(s))} \boldsymbol{\alpha}^{\prime} \operatorname{diag}(\boldsymbol{\phi}(s))\left\{\mathbb{I}_{N}-(\operatorname{diag}(\boldsymbol{\phi}(s) \circ(\mathbf{1}-\boldsymbol{\alpha})) \mathbf{G})^{\prime}\right\}^{-1} \mathbf{v} .
$$

We thus obtain the following equilibrium condition for the MRS and the MRT of aggregate consumption and labor:

$$
V^{\prime}(L(s))=\Lambda(s) U^{\prime}(C(s)) \frac{C(s)}{L(s)}
$$

with a labor wedge given by

$$
\Lambda(s)=\boldsymbol{\alpha}^{\prime} \operatorname{diag}(\boldsymbol{\phi}(s))\left\{\mathbb{I}_{N}-(\operatorname{diag}(\boldsymbol{\phi}(s) \circ(\mathbf{1}-\boldsymbol{\alpha})) \mathbf{G})^{\prime}\right\}^{-1} \mathbf{v}
$$

We may simplify our expression for the labor wedge as follows. First, we may write it as

$$
\Lambda(s)=\boldsymbol{\alpha}^{\prime}\left\{\left[\mathbb{I}_{N}-(\operatorname{diag}(\boldsymbol{\phi}(s)) \operatorname{diag}(\mathbf{1}-\boldsymbol{\alpha}) \mathbf{G})^{\prime}\right] \operatorname{diag}(\boldsymbol{\phi}(s))^{-1}\right\}^{-1} \mathbf{v}
$$

Or equivalently,

$$
\Lambda(s)=\boldsymbol{\alpha}^{\prime}\left\{\left[\mathbb{I}_{N}-\mathbf{G}^{\prime} \operatorname{diag}(\mathbf{1}-\boldsymbol{\alpha}) \operatorname{diag}(\boldsymbol{\phi}(s))\right] \operatorname{diag}(\boldsymbol{\phi}(s))^{-1}\right\}^{-1} \mathbf{v}
$$

which finally reduces to,

$$
\Lambda(s)=\boldsymbol{\alpha}^{\prime}\left[\operatorname{diag}(\boldsymbol{\phi}(s))^{-1}-\mathbf{G}^{\prime} \operatorname{diag}(\mathbf{1}-\boldsymbol{\alpha})\right]^{-1} \mathbf{v}
$$

as in (14). 
What remains to be shown is $\Lambda(\mathbf{1})=1$. Plugging $\boldsymbol{\phi}=\mathbf{1}$ into (14) gives us

$$
\Lambda(\mathbf{1})=\boldsymbol{\alpha}^{\prime}\left[\mathbb{I}_{N}-(\operatorname{diag}(\mathbf{1}-\boldsymbol{\alpha}) \mathbf{G})^{\prime}\right]^{-1} \mathbf{v}
$$

One can show that

$$
\boldsymbol{\alpha}^{\prime}\left[\mathbb{I}_{N}-(\operatorname{diag}(\mathbf{1}-\boldsymbol{\alpha}) \mathbf{G})^{\prime}\right]^{-1}=\mathbf{1}^{\prime}
$$

To see this, note that

$$
\begin{aligned}
\mathbf{1}^{\prime}\left[\mathbb{I}_{N}-(\operatorname{diag}(\mathbf{1}-\boldsymbol{\alpha}) \mathbf{G})^{\prime}\right] & =\mathbf{1}^{\prime}-\mathbf{1}^{\prime} \mathbf{G}^{\prime} \operatorname{diag}(\mathbf{1}-\boldsymbol{\alpha})=\mathbf{1}^{\prime}-\mathbf{1}^{\prime} \operatorname{diag}(\mathbf{1}-\boldsymbol{\alpha}) \\
& =\mathbf{1}^{\prime}-(\mathbf{1}-\boldsymbol{\alpha})^{\prime}=\boldsymbol{\alpha}^{\prime} .
\end{aligned}
$$

Therefore

$$
\boldsymbol{\alpha}^{\prime}\left[\mathbb{I}_{N}-(\operatorname{diag}(\mathbf{1}-\boldsymbol{\alpha}) \mathbf{G})^{\prime}\right]^{-1}=\mathbf{1}^{\prime}\left[\mathbb{I}_{N}-(\operatorname{diag}(\mathbf{1}-\boldsymbol{\alpha}) \mathbf{G})^{\prime}\right]\left[\mathbb{I}_{N}-(\operatorname{diag}(\mathbf{1}-\boldsymbol{\alpha}) \mathbf{G})^{\prime}\right]^{-1}=\mathbf{1}^{\prime}
$$

Substituting this into (77), we get that $\Lambda(\mathbf{1})=\mathbf{1}^{\prime} \mathbf{v}=1$.

Next we obtain our expression for aggregate TFP. We first take the production function in (7), rewritten here:

$$
q_{i}(s)=\psi_{i}(s) z_{i}(s) \ell_{i}(s)^{\alpha_{i}}\left(\prod_{j \in I} x_{i j}(s)^{g_{i j}}\right)^{1-\alpha_{i}}
$$

Dividing through by output $q_{i}(s)$ and using the fact that production exhibits constant returns to scale, we may express the production function in intensive form as follows:

$$
1=\psi_{i}(s) z_{i}(s)\left(\frac{\ell_{i}(s)}{q_{i}(s)}\right)^{\alpha_{i}}\left(\prod_{j \in I}\left(\frac{x_{i j}(s)}{q_{i}(s)}\right)^{g_{i j}}\right)^{1-\alpha_{i}} \forall i, s .
$$

From (8) and (9), we have that

$$
\frac{\ell_{i}(s)}{q_{i}(s)}=\frac{U^{\prime}(C(s)) C(s)}{V^{\prime}(L(s))} \phi_{i}(s) v_{i} \alpha_{i} \frac{1}{c_{i}(s)} \quad \forall i, s .
$$

and

$$
\frac{x_{i j}(s)}{q_{i}(s)}=\phi_{i}(s)\left(1-\alpha_{i}\right) g_{i j} \frac{v_{i}}{v_{j}} \frac{c_{j}(s)}{c_{i}(s)} \quad \forall i, j, s .
$$

Substituting these expressions into (78) we get

$$
1=z_{i}(s) \psi_{i}(s) \phi_{i}(s)\left(\alpha_{i} \frac{U^{\prime}(C(s)) C(s)}{V^{\prime}(L(s))} v_{i} \frac{1}{c_{i}(s)}\right)^{\alpha_{i}}\left(\prod_{j \in I}\left(\left(1-\alpha_{i}\right) g_{i j} \frac{v_{i}}{v_{j}} \frac{c_{j}(s)}{c_{i}(s)}\right)^{g_{i j}}\right)^{1-\alpha_{i}} \forall i, s .
$$


Therefore,

$$
c_{i}(s)=z_{i}(s) \psi_{i}(s) \phi_{i}(s) v_{i}\left(\alpha_{i} \frac{U^{\prime}(C(s)) C(s)}{V^{\prime}(L(s))}\right)^{\alpha_{i}}\left(\prod_{j \in I}\left(\left(1-\alpha_{i}\right) g_{i j} \frac{c_{j}(s)}{v_{j}}\right)^{g_{i j}}\right)^{1-\alpha_{i}} \forall i, s .
$$

Taking the logarithm and rearranging the above expression gives us $\log c_{i}(s)-\left(1-\alpha_{i}\right) \sum_{j \in I} g_{i j} \log c_{j}(s)=\log z_{i}(s)+\log \psi_{i}(s)+\log \phi_{i}(s)+\alpha_{i} \log \left(\frac{U^{\prime}(C(s)) C(s)}{V^{\prime}(L(s))}\right)+\log K_{i}$,

where

$$
\log K_{i}=\log v_{i}+\alpha_{i} \log \alpha_{i}+\left(1-\alpha_{i}\right) \log \left(1-\alpha_{i}\right)-\left(1-\alpha_{i}\right) \sum_{j} g_{i j}\left(\log g_{i j}-\log v_{j}\right)
$$

Stacking equation (79) into matrix form gives us

$$
\left(\mathbb{I}_{N}-\operatorname{diag}(\mathbf{1}-\boldsymbol{\alpha}) \mathbf{G}\right) \log \mathbf{c}(s)=\log \mathbf{z}(s)+\log \boldsymbol{\psi}(s)+\log \boldsymbol{\phi}(s)+\boldsymbol{\alpha} \log \left(\frac{U^{\prime}(C(s)) C(s)}{V^{\prime}(L(s))}\right)+\log \mathbf{K} .
$$

where the elements of $\log \mathbf{K}$ are those given in (80). Solving the above equation for the consumption vector we get

$$
\log \mathbf{c}(s)=\mathbf{B}(\log \mathbf{z}(s)+\log \boldsymbol{\psi}(s)+\log \boldsymbol{\phi}(s))+\mathbf{B} \boldsymbol{\alpha} \log \left(\frac{U^{\prime}(C(s)) C(s)}{V^{\prime}(L(s))}\right)+\mathbf{B} \log \mathbf{K} .
$$

where $\mathbf{B} \equiv\left[\mathbb{I}_{N}-\operatorname{diag}(\mathbf{1}-\boldsymbol{\alpha}) \mathbf{G}\right]^{-1}$ is the Leontief inverse matrix.

Finally we may substitute (81) into the household's Cobb-Douglas consumption aggregator, $\log C(s)=\mathbf{v}^{\prime} \log \boldsymbol{c}(s)$. This gives us

$$
\log C(s)=\mathbf{v}^{\prime} \mathbf{B}(\log \mathbf{z}(s)+\log \psi(s)+\log \phi(s))+\mathbf{v}^{\prime} \mathbf{B} \boldsymbol{\alpha} \log \left(\frac{U^{\prime}(C(s)) C(s)}{V^{\prime}(L(s))}\right)+\mathbf{v}^{\prime} \mathbf{B} \log \mathbf{K}
$$

Note that $\mathbf{B} \boldsymbol{\alpha}=\mathbf{1}$, so that $\mathbf{v}^{\prime} \mathbf{B} \boldsymbol{\alpha}=\mathbf{v}^{\prime} \mathbf{1}=1$. Therefore, equation (82) reduces to

$$
\log C(s)=\mathbf{v}^{\prime} \mathbf{B}(\log \mathbf{z}(s)+\log \boldsymbol{\psi}(s)+\log \phi(s))+\log \left(\frac{U^{\prime}(C(s)) C(s)}{V^{\prime}(L(s))}\right)+\mathbf{v}^{\prime} \mathbf{B} \log \mathbf{K} .
$$

Finally we combine (83) with our equilibrium aggregate condition in (76). Taking logs of equation (76) we have

$$
-\log \Lambda(s)+\log L(s)=\log \left(\frac{U^{\prime}(C(s)) C(s)}{V^{\prime}(L(s))}\right)
$$


Substituting this into (83) we get

$$
\log C(s)=\mathbf{v}^{\prime} \mathbf{B}(\log \mathbf{z}(s)+\log \boldsymbol{\psi}(s)+\log \boldsymbol{\phi}(s))-\log \Lambda(s)+\log L(s)+\mathbf{v}^{\prime} \mathbf{B} \log \mathbf{K}
$$

Therefore

$$
C(s)=\kappa A(\mathbf{z}(s)) A(\boldsymbol{\psi}(s)) A(\boldsymbol{\phi}(s)) \Lambda(s)^{-1} L(s),
$$

where $A(\cdot)$ is a function defined in (16) and $\kappa \equiv \exp \left(\mathbf{v}^{\prime} \mathbf{B} \log \mathbf{K}\right)$ is a scalar. It is immediate that $\log A(\mathbf{1})=\mathbf{v}^{\prime} \mathbf{B} \log \mathbf{1}=0$. Therefore $A(\mathbf{1})=1$. QED.

Proof of Corollary 1. From Theorem 1, $\boldsymbol{\phi}=\mathbf{1}$ implies $\Lambda(\mathbf{1})=1$ and $A(\mathbf{1})=1$. QED.

Proof of Theorem 2. Part (i). To a first-order approximation around the efficiency,

$$
d \log \Phi(s)=\left[\left.\frac{d \log A(\phi(s))}{d \log \phi_{i}}\right|_{\phi=1}-\left.\frac{d \log \Lambda(s)}{d \log \phi_{i}}\right|_{\phi=1}\right] d \log \phi_{i} .
$$

The first term on the right hand side of equation (84) is straightforward, given by

$$
\frac{d \log A(\boldsymbol{\phi}(s))}{d \log \phi_{i}}=\boldsymbol{v}^{\prime} \mathbf{B} e_{i}=\boldsymbol{v}^{\prime}\left[\mathbb{I}_{N}-\operatorname{diag}(\mathbf{1}-\boldsymbol{\alpha}) \mathbf{G}\right]^{-1} e_{i}
$$

This term is log-linear and hence holds globally rather than just locally at efficiency $(\boldsymbol{\phi}=\mathbf{1})$.

The second term on the right hand side of equation (84) is not log-linear. We may write it as:

$$
\frac{d \log \Lambda(s)}{d \log \phi_{i}}=-\Lambda(s)^{-1} \frac{d \Lambda(s)}{d \log \phi_{i}}
$$

where

$$
\Lambda(s)=\boldsymbol{\alpha}^{\prime}\left\{\operatorname{diag}(\boldsymbol{\phi}(s))^{-1}-(\operatorname{diag}(\mathbf{1}-\boldsymbol{\alpha}) \mathbf{G})^{\prime}\right\}^{-1} \boldsymbol{v}
$$

Thus we have that the second term is given by:

$$
\frac{d \log \Lambda(s)}{d \log \phi_{i}}=-\Lambda(s)^{-1} \boldsymbol{\alpha}^{\prime} \frac{d\left\{\operatorname{diag}(\boldsymbol{\phi}(s))^{-1}-(\operatorname{diag}(\mathbf{1}-\boldsymbol{\alpha}) \mathbf{G})^{\prime}\right\}^{-1}}{d \log \phi_{i}} \boldsymbol{v}
$$

Next, we use the fact that for any invertible matrix $A$, the following is true: $\frac{d A^{-1}}{d x}=-A^{-1} \frac{d A}{d x} A^{-1}$. 
Applying this, we have that

$$
\begin{aligned}
\frac{d\left\{\operatorname{diag}(\boldsymbol{\phi}(s))^{-1}-(\operatorname{diag}(\mathbf{1}-\boldsymbol{\alpha}) \mathbf{G})^{\prime}\right\}^{-1}}{d \log \phi_{i}}= & -\left\{\operatorname{diag}(\boldsymbol{\phi}(s))^{-1}-(\operatorname{diag}(\mathbf{1}-\boldsymbol{\alpha}) \mathbf{G})^{\prime}\right\}^{-1} \\
& \times \frac{d\left\{\operatorname{diag}(\boldsymbol{\phi}(s))^{-1}-(\operatorname{diag}(\mathbf{1}-\boldsymbol{\alpha}) \mathbf{G})^{\prime}\right\}}{d \log \phi_{i}} \\
& \times\left\{\operatorname{diag}(\boldsymbol{\phi}(s))^{-1}-(\operatorname{diag}(\mathbf{1}-\boldsymbol{\alpha}) \mathbf{G})^{\prime}\right\}^{-1}
\end{aligned}
$$

Further note that:

$$
\begin{aligned}
\frac{d\left\{\operatorname{diag}(\boldsymbol{\phi}(s))^{-1}-(\operatorname{diag}(\mathbf{1}-\boldsymbol{\alpha}) \mathbf{G})^{\prime}\right\}}{d \log \phi_{i}} & =\frac{d\left\{\operatorname{diag}(\exp (\log \boldsymbol{\phi}(s)))^{-1}\right\}}{d \log \phi_{i}}=\operatorname{diag}\left(e_{i}\right) \operatorname{diag}(\exp (\log \boldsymbol{\phi}(s)))^{-1} \\
& =\operatorname{diag}\left(e_{i}\right) \operatorname{diag}(\boldsymbol{\phi}(s))^{-1}
\end{aligned}
$$

Plugging this into (86) gives us,

$$
\begin{aligned}
\frac{d \log \Lambda(s)}{d \log \phi_{i}}= & \Lambda(s)^{-1} \boldsymbol{\alpha}^{\prime}\left\{\operatorname{diag}(\boldsymbol{\phi}(s))^{-1}-(\operatorname{diag}(\mathbf{1}-\boldsymbol{\alpha}) \mathbf{G})^{\prime}\right\}^{-1} \\
& \times \operatorname{diag}\left(e_{i}\right) \operatorname{diag}(\boldsymbol{\phi}(s))^{-1}\left\{\operatorname{diag}(\boldsymbol{\phi}(s))^{-1}-(\operatorname{diag}(\mathbf{1}-\boldsymbol{\alpha}) \mathbf{G})^{\prime}\right\}^{-1} \boldsymbol{v}
\end{aligned}
$$

Evaluating this expression at the efficient benchmark, $\phi=\mathbf{1}$,

$$
\left.\frac{d \log \Lambda(s)}{d \log \phi_{i}}\right|_{\phi=\mathbf{1}}=\boldsymbol{\alpha}^{\prime}\left\{\mathbb{I}_{N}-(\operatorname{diag}(\mathbf{1}-\boldsymbol{\alpha}) \mathbf{G})^{\prime}\right\}^{-1} \operatorname{diag}\left(e_{i}\right) \mathbb{I}_{N}\left\{\mathbb{I}_{N}-(\operatorname{diag}(\mathbf{1}-\boldsymbol{\alpha}) \mathbf{G})^{\prime}\right\}^{-1} \boldsymbol{v}
$$

where $\Lambda(\mathbf{1})^{-1}=1$. Simplifying,

$$
\left.\frac{d \log \Lambda(s)}{d \log \phi_{i}}\right|_{\boldsymbol{\phi}=\mathbf{1}}=\boldsymbol{\alpha}^{\prime}\left\{\mathbb{I}_{N}-(\operatorname{diag}(\mathbf{1}-\boldsymbol{\alpha}) \mathbf{G})^{\prime}\right\}^{-1} \operatorname{diag}\left(e_{i}\right)\left\{\mathbb{I}_{N}-(\operatorname{diag}(\mathbf{1}-\boldsymbol{\alpha}) \mathbf{G})^{\prime}\right\}^{-1} \boldsymbol{v}
$$

Next we use the fact that

$$
\boldsymbol{\alpha}^{\prime}\left[\mathbb{I}_{N}-(\operatorname{diag}(\mathbf{1}-\boldsymbol{\alpha}) \mathbf{G})^{\prime}\right]^{-1}=\mathbf{1}^{\prime}
$$

This gives us the following simplified expression:

$$
\left.\frac{d \log \Lambda(s)}{d \log \phi_{i}}\right|_{\phi=\mathbf{1}}=\boldsymbol{e}_{i}^{\prime}\left\{\mathbb{I}_{N}-(\operatorname{diag}(\mathbf{1}-\boldsymbol{\alpha}) \mathbf{G})^{\prime}\right\}^{-1} \boldsymbol{v}
$$

Note that the expression in (87) is a scalar. Because it is a scalar, it must be equal to its transpose:

$$
\boldsymbol{e}_{i}^{\prime}\left\{\mathbb{I}_{N}-(\operatorname{diag}(\mathbf{1}-\boldsymbol{\alpha}) \mathbf{G})^{\prime}\right\}^{-1} \boldsymbol{v}=\boldsymbol{v}^{\prime}\left[\mathbb{I}_{N}-\operatorname{diag}(\mathbf{1}-\boldsymbol{\alpha}) \mathbf{G}\right]^{-1} \boldsymbol{e}_{i}
$$


Therefore

$$
\left.\frac{d \log \Lambda(s)}{d \log \phi_{i}}\right|_{\phi=\mathbf{1}}=\boldsymbol{v}^{\prime}\left[\mathbb{I}_{N}-\operatorname{diag}(\mathbf{1}-\boldsymbol{\alpha}) \mathbf{G}\right]^{-1} \boldsymbol{e}_{i}=\boldsymbol{v}^{\prime} \mathbf{B} e_{i}
$$

Finally, substituting expressions (85) and (88) into (84) gives us

$$
\left.\frac{d \log \Phi(s)}{d \log \phi_{i}}\right|_{\phi=\mathbf{1}}=\boldsymbol{v}^{\prime} \mathbf{B} e_{i}-\boldsymbol{v}^{\prime} \mathbf{B} e_{i}
$$

Therefore, to a first-order approximation around the efficient benchmark

$$
\left.\frac{d \log \Phi(s)}{d \log \phi_{i}}\right|_{\phi=1}=0
$$

for all $i$.

Part (ii). This follows directly from part (i) and (84). QED.

Proof of Corollary 2. From Hulten (1978), in an efficient economy

$$
\left.\frac{d \log A(\mathbf{z}(s))}{d \log z_{i}}\right|_{\phi=1}=\lambda_{i}
$$

where the Domar weight is defined as equilibrium sales over GDP, $\lambda_{i} \equiv p_{i} y_{i} / C$. From Part (ii) of Theorem 2,

$$
\left.\frac{d \log \Lambda(s)}{d \log \phi_{i}}\right|_{\phi=\mathbf{1}}=\left.\frac{d \log A(\phi(s))}{d \log \phi_{i}}\right|_{\phi=\mathbf{1}}=\left.\frac{d \log A(\mathbf{z}(s))}{d \log z_{i}}\right|_{\phi=\mathbf{1}} .
$$

Therefore,

$$
\left.\frac{d \log \Lambda(s)}{d \log \phi_{i}}\right|_{\phi=1}=\lambda_{i}
$$

QED.

Proof of Proposition 7. From market clearing, total sales for each sector must satisfy

$$
p_{i}(s) q_{i}(s)=p_{i}(s) c_{i}(s)+p_{i}(s) \sum_{j \in I} x_{j i}(s), \forall i \in I
$$

Let $b_{i}(s)$ denote total expenditures in sector $i$ :

$$
b_{i}(s)=w_{i}(s) \ell_{i}(s)+\sum_{j} p_{j}(s) x_{i j}(s)
$$


Then due to the Cobb-Douglas structure

$$
p_{i}(s) x_{j i}(s)=\left(1-\alpha_{j}\right) g_{j i} b_{j}(s)
$$

and due to the Cobb-Douglas preferences:

$$
p_{i}(s) c_{i}(s)=v_{i} u_{0}(s)
$$

where $u_{0}$ denotes total household expenditure (equivalently, household wealth). Thus Cobb-Douglas structure of preferences and technologies implies constant expenditure shares of firms and households over the commodities.

Combining the constant expenditure shares from conditions (90) and (91) with the market clearing condition in (89) yields the following equation

$$
m_{i}(s)=v_{i} u_{0}(s)+\sum_{j \in I}\left(1-\alpha_{j}\right) g_{j i} b_{j}(s), \text { for all } i,
$$

where $m_{i}(s)=p_{i}(s) q_{i}(s)$. Stacking equation (92) for each sector $i$ atop one another, we have

$$
\left[\begin{array}{c}
m_{1}(s) \\
m_{2}(s) \\
\vdots \\
m_{N}(s)
\end{array}\right]=\left[\begin{array}{c}
v_{1} \\
v_{2} \\
\vdots \\
v_{N}
\end{array}\right] u_{0}(s)+\left(\left[\begin{array}{cccc}
1-\alpha_{1} & 1-\alpha_{2} & \cdots & 1-\alpha_{N} \\
1-\alpha_{1} & 1-\alpha_{2} & & \\
\vdots & & \ddots & \\
1-\alpha_{1} & 1-\alpha_{2} & & 1-\alpha_{N}
\end{array}\right] \circ\left[\begin{array}{cccc}
g_{11} & g_{21} & \cdots & g_{N 1} \\
g_{12} & g_{22} & \\
\vdots & & \ddots \\
g_{1 N} & & g_{N N}
\end{array}\right]\right)\left[\begin{array}{c}
b_{1}(s) \\
b_{2} \\
\vdots \\
b_{N}
\end{array}\right]
$$

where $\circ$ denotes the Hadamard (entrywise) product. We thus write the system of equations in (93) as follows

$$
\mathbf{m}(s)=\mathbf{v} u_{0}(s)+(\operatorname{diag}(\mathbf{1}-\alpha) \mathbf{G})^{\prime} \mathbf{b}(s),
$$

where $\mathbf{m}(s) \equiv \operatorname{diag}(\mathbf{p}(s)) \mathbf{q}(s)$ denotes the equilibrium vector of sales. Thus, using market clearing and the optimality of expenditure on each good, we can relate total revenue of each firm to the total expenditures of each sector and to the expenditure of the household.

Next, noting that $\pi_{i}(s)=\left(1-\tau_{i}(s)\right) m_{i}(s)-b_{i}(s)$, total household expenditure (equivalently, wealth) is given by

$$
u_{0}(s)=L(s)+\sum_{i=1}^{N}\left(\left(1-\tau_{i}(s)\right) m_{i}(s)-b_{i}(s)\right)+\sum_{i=1}^{N} \tau_{i} g_{i}(s) .
$$


Therefore household expenditure may be written as

$$
u_{0}(s)=L(s)+\sum_{i=1}^{N}\left(m_{i}(s)-b_{i}(s)\right) .
$$

That is, the household's non-labor income is equal to the difference between total expenditures and total sales. We rewrite this in vector form as follows

$$
u_{0}(s)=L(s)+\mathbf{1}^{\prime}(\mathbf{m}(s)-\mathbf{b}(s)) .
$$

Using (96) to substitute for $u_{0}(s)$ into our market clearing condition (94), we obtain the following fixed point in sectoral sales and sectoral expenditure:

$$
\mathbf{m}(s)=\mathbf{v} L(s)+\mathbf{v} \mathbf{1}^{\prime}(\mathbf{m}(s)-\mathbf{b}(s))+(\operatorname{diag}(\mathbf{1}-\alpha) \mathbf{G})^{\prime} \mathbf{b}(s) .
$$

From firm optimality, we have that firm expenditures satisfy $b_{i}(s)=\phi_{i}(s) m_{i}(s)$. Stacking this equation atop one another sector-by-sector, we have

$$
\mathbf{b}(s)=\operatorname{diag}(\boldsymbol{\phi}(s)) \mathbf{m}(s) .
$$

Substituting (98) for $\mathbf{b}(s)$ into (97), yields

$$
\mathbf{m}(s)=\mathbf{v} L(s)+\mathbf{v} \mathbf{1}^{\prime}(\mathbf{m}(s)-\operatorname{diag}(\boldsymbol{\phi}(s)) \mathbf{m}(s))+(\operatorname{diag}(\mathbf{1}-\alpha) \mathbf{G})^{\prime} \operatorname{diag}(\boldsymbol{\phi}(s)) \mathbf{m}(s)
$$

With some algebraic manipulation we may re-write this as

$$
\mathbf{m}(s)=\left[\mathbf{v} \mathbf{1}^{\prime}\left(\mathbb{I}_{N}-\operatorname{diag}(\boldsymbol{\phi}(s))\right)+(\operatorname{diag}(\mathbf{1}-\alpha) \mathbf{G})^{\prime} \operatorname{diag}(\phi(s))\right] \mathbf{m}(s)+\mathbf{v} L(s)
$$

Total revenue thereby satisfies

$$
\mathbf{m}(s)=\left[\mathbb{I}_{N}-(\operatorname{diag}(\mathbf{1}-\alpha) \mathbf{G})^{\prime} \operatorname{diag}(\boldsymbol{\phi}(s))-\mathbf{v} \mathbf{1}^{\prime}\left(\mathbb{I}_{N}-\operatorname{diag}(\boldsymbol{\phi}(s))\right)\right]^{-1} \mathbf{v} L(s)
$$

This equation gives us (40), with the vector function $\Omega$ defined in (41). QED. 


\section{Equilibrium Consumption and Labor}

Lemma 4. Suppose utility of consumption and labor is homothetic and given by (35). Then, equilibrium aggregate labor and consumption (GDP) are given by the following functions, respectively:

$$
\begin{aligned}
\log L(s) & =\frac{1-\gamma}{\epsilon+\gamma} \log (A(\mathbf{z}(s)) A(\boldsymbol{\psi}(s)) A(\boldsymbol{\phi}(s)))+\frac{\gamma}{\epsilon+\gamma} \log \Lambda(s) \\
\log C(s) & =\frac{1+\epsilon}{\epsilon+\gamma} \log (A(\mathbf{z}(s)) A(\boldsymbol{\psi}(s)) A(\boldsymbol{\phi}(s)))-\frac{\epsilon}{\epsilon+\gamma} \log \Lambda(s) .
\end{aligned}
$$

Lemma 4 provides explicit formulas for equilibrium consumption (GDP) and labor in this economy. As we have emphasized throughout, sectoral distortions affect these aggregates through two channels: via movements in TFP and movements in the aggregate labor wedge. While TFP and the aggregate labor wedge do not depend on $\epsilon$ and $\gamma$, clearly equilibrium labor and consumption are sensitive to these parameters.

Proof of Lemma 4. With the homothetic preferences stated in (35), we have the following system of two equations and two unknowns:

$$
\begin{aligned}
& L(s)^{\epsilon}=\Lambda(s) C(s)^{-\gamma} \frac{C(s)}{L(s)} \\
& C(s)=A(\mathbf{z}(s)) A(\boldsymbol{\psi}(s)) A(\phi(s)) \Lambda(s)^{-1} L(s)
\end{aligned}
$$

Substituting expression (102) for consumption into (101), we get

$$
L(s)^{1+\epsilon}=\Lambda(s)\left(A(\mathbf{z}(s)) A(\boldsymbol{\psi}(s)) A(\phi(s)) \Lambda(s)^{-1} L(s)\right)^{1-\gamma}
$$

Solving this expression for $L(s)$,

$$
L(s)^{\epsilon+\gamma}=\Lambda(s)^{\gamma}(A(\mathbf{z}(s)) A(\boldsymbol{\psi}(s)) A(\phi(s)))^{1-\gamma},
$$

and taking logs of both sides gives us equilibrium aggregate labor

$$
\log L(s)=\frac{1-\gamma}{\epsilon+\gamma} \log (A(\mathbf{z}(s)) A(\boldsymbol{\psi}(s)) A(\phi(s)))+\frac{\gamma}{\epsilon+\gamma} \log \Lambda(s)
$$

as in (99). Finally, substituting this back into the aggregate production function (102) yields the following expression for equilibrium aggregate consumption

$$
\log C(s)=\frac{1+\epsilon}{\epsilon+\gamma} \log (A(\mathbf{z}(s)) A(\boldsymbol{\psi}(s)) A(\phi(s)))-\frac{\epsilon}{\epsilon+\gamma} \log \Lambda(s)
$$


as in (100). QED.

\section{Appendix B: Proofs for the Simple Examples}

Proof of Proposition 3. In the vertical economy, the vector of labor shares is $\boldsymbol{\alpha}=(1,0,0)^{\prime}$ and the vector of household consumption shares is $\mathbf{v}=(0,0,1)^{\prime}$. The input-output matrix is given by

$$
\mathbf{G}=\left[\begin{array}{lll}
0 & 0 & 0 \\
1 & 0 & 0 \\
0 & 1 & 0
\end{array}\right]
$$

Note that while the entries in the first row of this matrix do not add up to one, this is a non-issue because firm 1's labor share is 1 (and hence its input share is zero).

We first compute the influence vector given by

$$
\mathbf{v}^{\prime}\left[\mathbb{I}_{3}-\operatorname{diag}(\mathbf{1}-\boldsymbol{\alpha}) \mathbf{G}\right]^{-1}
$$

where $\mathbf{1}-\boldsymbol{\alpha}=(0,1,1)^{\prime}$. Substituting in this economy's values for $\mathbf{v}, \alpha$, and $\mathbf{G}$, into (104) give us

$$
\mathbf{v}^{\prime}\left[\mathbb{I}_{3}-\operatorname{diag}(\mathbf{1}-\boldsymbol{\alpha}) \mathbf{G}\right]^{-1}=\left[\begin{array}{lll}
0 & 0 & 1
\end{array}\right]\left(\left[\begin{array}{lll}
1 & 0 & 0 \\
0 & 1 & 0 \\
0 & 0 & 1
\end{array}\right]-\left[\begin{array}{lll}
0 & 0 & 0 \\
0 & 1 & 0 \\
0 & 0 & 1
\end{array}\right]\left[\begin{array}{lll}
0 & 0 & 0 \\
1 & 0 & 0 \\
0 & 1 & 0
\end{array}\right]\right)^{-1}
$$

As a result,

$$
\mathbf{v}^{\prime}\left[\mathbb{I}_{3}-\operatorname{diag}(\mathbf{1}-\boldsymbol{\alpha}) \mathbf{G}\right]^{-1}=\left[\begin{array}{lll}
1 & 1 & 1
\end{array}\right]
$$

This implies

$$
\log A(\phi(s))=\left[\begin{array}{lll}
1 & 1 & 1
\end{array}\right] \log \boldsymbol{\phi}(s) \text { and } \log A(\boldsymbol{\psi}(s))=\left[\begin{array}{lll}
1 & 1 & 1
\end{array}\right] \log \boldsymbol{\psi}(s)
$$

and therefore

$$
A(\phi(s))=\phi_{1}(s) \phi_{2}(s) \phi_{3}(s) \text { and } A(\boldsymbol{\psi}(s))=\psi_{1}(s) \psi_{2}(s) \psi_{3}(s)
$$

As for the labor wedge, we have

$$
\Lambda(s) \equiv \boldsymbol{\alpha}^{\prime}\left\{\operatorname{diag}(\boldsymbol{\phi}(s))^{-1}-(\operatorname{diag}(\mathbf{1}-\boldsymbol{\alpha}) \mathbf{G})^{\prime}\right\}^{-1} \boldsymbol{v}
$$


Substituting in this economy's values for $\boldsymbol{\alpha}$ and $\mathbf{G}$, we obtain the following expression:

$$
\operatorname{diag}(\phi(s))^{-1}-(\operatorname{diag}(\mathbf{1}-\boldsymbol{\alpha}) \mathbf{G})^{\prime}=\left[\begin{array}{ccc}
\frac{1}{\phi_{1}(s)} & 0 & 0 \\
0 & \frac{1}{\phi_{2}(s)} & 0 \\
0 & 0 & \frac{1}{\phi_{3}(s)}
\end{array}\right]-\left[\begin{array}{ccc}
0 & 1 & 0 \\
0 & 0 & 1 \\
0 & 0 & 0
\end{array}\right]=\left[\begin{array}{ccc}
\frac{1}{\phi_{1}(s)} & -1 & 0 \\
0 & \frac{1}{\phi_{2}(s)} & -1 \\
0 & 0 & \frac{1}{\phi_{3}(s)}
\end{array}\right]
$$

Taking the inverse of this expression gives us:

$$
\left[\begin{array}{ccc}
\frac{1}{\phi_{1}(s)} & -1 & 0 \\
0 & \frac{1}{\phi_{2}(s)} & -1 \\
0 & 0 & \frac{1}{\phi_{3}(s)}
\end{array}\right]^{-1}=\left[\begin{array}{ccc}
\phi_{1}(s) & \phi_{1}(s) \phi_{2}(s) & \phi_{1}(s) \phi_{2}(s) \phi_{3}(s) \\
0 & \phi_{2}(s) & \phi_{2}(s) \phi_{3}(s) \\
0 & 0 & \phi_{3}(s)
\end{array}\right]
$$

Therefore

$$
\Lambda(s)=\left[\begin{array}{lll}
1 & 0 & 0
\end{array}\right]\left[\begin{array}{ccc}
\phi_{1}(s) & \phi_{1}(s) \phi_{2}(s) & \phi_{1}(s) \phi_{2}(s) \phi_{3}(s) \\
0 & \phi_{2}(s) & \phi_{2}(s) \phi_{3}(s) \\
0 & 0 & \phi_{3}(s)
\end{array}\right]\left[\begin{array}{l}
0 \\
0 \\
1
\end{array}\right]=\phi_{1}(s) \phi_{2}(s) \phi_{3}(s)
$$

as in (26). Finally, combining this with (105) gives us TFP as in (26). QED.

Proof of Proposition 4. In the horizontal economy, the vector of labor shares is $\boldsymbol{\alpha}=(1,1,1)^{\prime}$ and the vector of household consumption shares is $\mathbf{v}=\left(v_{1}, v_{2}, v_{3}\right)^{\prime}$. The input-output matrix is given by

$$
\mathbf{G}=\left[\begin{array}{lll}
0 & 0 & 0 \\
0 & 0 & 0 \\
0 & 0 & 0
\end{array}\right]
$$

All entries in the input-output matrix are zero as no commodity is bought by any firm as an input. ${ }^{40}$

We compute the influence vector from equation (104). Substituting in this economy's values for $\mathbf{v}, \boldsymbol{\alpha}$, and $\mathbf{G}$, we obtain the following expression for the influence vector:

$$
\mathbf{v}^{\prime}\left[\mathbb{I}_{3}-\operatorname{diag}(\mathbf{1}-\boldsymbol{\alpha}) \mathbf{G}\right]^{-1}=\left[\begin{array}{lll}
v_{1} & v_{2} & v_{3}
\end{array}\right] .
$$

This implies

$$
\log A(\phi(s))=\left[\begin{array}{lll}
v_{1} & v_{2} & v_{3}
\end{array}\right] \log \boldsymbol{\phi}(s) \text { and } \log A(\boldsymbol{\psi}(s))=\left[\begin{array}{lll}
v_{1} & v_{2} & v_{3}
\end{array}\right] \log \boldsymbol{\psi}(s)
$$

\footnotetext{
${ }^{40}$ Again, note that for all rows, the entries do not add up to one. This again does not matter because all firms have a labor share of 1 .
} 
and therefore

$$
A(\phi(s))=\phi_{1}(s)^{v_{1}} \phi_{2}(s)^{v_{2}} \phi_{3}(s)^{v_{3}} \text { and } A(\boldsymbol{\psi}(s))=\psi_{1}(s)^{v_{1}} \psi_{2}(s)^{v_{2}} \psi_{3}(s)^{v_{3}}
$$

As for $\Lambda(\phi)$, we have

$$
\Lambda(s) \equiv \boldsymbol{\alpha}^{\prime}\left\{\operatorname{diag}(\boldsymbol{\phi}(s))^{-1}-(\operatorname{diag}(\mathbf{1}-\boldsymbol{\alpha}) \mathbf{G})^{\prime}\right\}^{-1} \boldsymbol{v}
$$

Substituting in this economy's values for $\boldsymbol{\alpha}$ and $\mathbf{G}$, we obtain the following expression:

$$
\operatorname{diag}(\phi(s))^{-1}-(\operatorname{diag}(\mathbf{1}-\boldsymbol{\alpha}) \mathbf{G})^{\prime}=\left[\begin{array}{ccc}
\frac{1}{\phi_{1}(s)} & 0 & 0 \\
0 & \frac{1}{\phi_{2}(s)} & 0 \\
0 & 0 & \frac{1}{\phi_{3}(s)}
\end{array}\right]
$$

Taking the inverse of this expression gives us:

$$
\left[\begin{array}{ccc}
\frac{1}{\phi_{1}(s)} & 0 & 0 \\
0 & \frac{1}{\phi_{2}(s)} & 0 \\
0 & 0 & \frac{1}{\phi_{3}(s)}
\end{array}\right]^{-1}=\left[\begin{array}{ccc}
\phi_{1}(s) & 0 & 0 \\
0 & \phi_{2}(s) & 0 \\
0 & 0 & \phi_{3}(s)
\end{array}\right]
$$

Therefore

$$
\Lambda(s)=\left[\begin{array}{lll}
1 & 1 & 1
\end{array}\right]\left[\begin{array}{ccc}
\phi_{1}(s) & 0 & 0 \\
0 & \phi_{2}(s) & 0 \\
0 & 0 & \phi_{3}(s)
\end{array}\right]\left[\begin{array}{c}
v_{1} \\
v_{2} \\
v_{3}
\end{array}\right]=v_{1} \phi_{1}(s)+v_{2} \phi_{2}(s)+v_{3} \phi_{2}(s)
$$

as in (28). Finally, combining this with (107) gives us TFP as in (27). QED.

Proof of Proposition 5. The vectors of labor shares and of input shares in this economy are given by

$$
\boldsymbol{\alpha}=\left[\begin{array}{c}
\alpha^{0} \\
\alpha^{1} \\
\vdots \\
\alpha^{N-1}
\end{array}\right]=\left[\begin{array}{c}
1 \\
\alpha \\
\vdots \\
\alpha^{N-1}
\end{array}\right], \operatorname{and} \mathbf{1}-\boldsymbol{\alpha}=\left[\begin{array}{c}
0 \\
1-\alpha \\
\vdots \\
1-\alpha^{N-1}
\end{array}\right]
$$

respectively. The vector of household consumption shares is given by

$$
\mathbf{v}^{\prime}=\frac{1}{\xi}\left[\begin{array}{llll}
\rho^{N-1} & \cdots & \rho^{1} & \rho^{0}
\end{array}\right]=\frac{1}{\xi}\left[\begin{array}{llll}
\rho^{N-1} & \cdots & \rho^{1} & 1
\end{array}\right],
$$


and the input-output matrix is given by

$$
\mathbf{G}=\left[\begin{array}{ccccc}
0 & 0 & 0 & \cdots & 0 \\
1 & 0 & 0 & \cdots & 0 \\
0 & 1 & 0 & \cdots & 0 \\
\vdots & & \ddots & & \vdots \\
0 & \cdots & 0 & 1 & 0
\end{array}\right]
$$

We first compute the influence vector given by (104). Substituting in this economy's values for $\boldsymbol{\alpha}$ and $\mathbf{G}$, we obtain the following expression:

$$
\mathbb{I}_{N}-\operatorname{diag}(\mathbf{1}-\boldsymbol{\alpha}) \mathbf{G}=\left[\begin{array}{ccccc}
1 & 0 & 0 & \cdots & 0 \\
-(1-\alpha) & 1 & 0 & \cdots & 0 \\
0 & -\left(1-\alpha^{2}\right) & 1 & \ldots & 0 \\
\vdots & & \ddots & \ddots & \vdots \\
0 & \cdots & 0 & -\left(1-\alpha^{N-1}\right) & 1
\end{array}\right]
$$

Taking the inverse of this expression we get

$$
\begin{gathered}
\chi_{1}(s)>\chi_{2}(s)>\cdots>\chi_{N}(s) \\
{\left[\mathbb{I}_{N}-\operatorname{diag}(\mathbf{1}-\boldsymbol{\alpha}) \mathbf{G}\right]^{-1}=\left[\begin{array}{ccccc}
1 & 0 & 0 & \cdots & 0 \\
\prod_{m=1}^{1}\left(1-\alpha^{m}\right) & 1 & 0 & \cdots & 0 \\
\prod_{m=1}^{2}\left(1-\alpha^{m}\right) & \prod_{m=2}^{2}\left(1-\alpha^{m}\right) & 1 & \cdots & 0 \\
\vdots & \cdots & \cdots & \ddots & \vdots \\
\prod_{m=1}^{N-1}\left(1-\alpha^{m}\right) & \cdots & \cdots & \prod_{m=N-1}^{N-1}\left(1-\alpha^{m}\right) & 1
\end{array}\right]}
\end{gathered}
$$

Therefore

$$
A(\phi(s))=\prod_{i=1}^{N} \phi_{i}(s)^{a_{i}}
$$

where the coefficients $a_{i}$ satisfy

$$
a_{i}=\frac{1}{\xi}\left(\rho^{N-i}+\sum_{j=i+1}^{N} \rho^{N-j}\left[\prod_{m=i}^{j-1}\left(1-\alpha^{m}\right)\right]\right) .
$$


Next consider the labor wedge.

$$
\operatorname{diag}(\phi(s))^{-1}-\mathbf{G}^{\prime} \operatorname{diag}(\mathbf{1}-\boldsymbol{\alpha})=\left[\begin{array}{ccccc}
\frac{1}{\phi_{1}(s)} & -(1-\alpha) & 0 & \cdots & 0 \\
0 & \frac{1}{\phi_{2}(s)} & -\left(1-\alpha^{2}\right) & \cdots & 0 \\
0 & 0 & \ddots & \ddots & 0 \\
\vdots & & \ddots & \frac{1}{\phi_{N-1}(s)} & -\left(1-\alpha^{N-1}\right) \\
0 & \cdots & 0 & 0 & \frac{1}{\phi_{N}(s)}
\end{array}\right]
$$

Therefore

$$
\Lambda(s)=\boldsymbol{\alpha}^{\prime}\left[\begin{array}{ccccc}
\frac{1}{\phi_{1}(s)} & -(1-\alpha) & 0 & \cdots & 0 \\
0 & \frac{1}{\phi_{2}(s)} & -\left(1-\alpha^{2}\right) & \cdots & 0 \\
0 & 0 & \ddots & \ddots & 0 \\
\vdots & & \ddots & \frac{1}{\phi_{N-1}(s)} & -\left(1-\alpha^{N-1}\right) \\
0 & \cdots & 0 & 0 & \frac{1}{\phi_{N}(s)}
\end{array}\right]^{-1} \mathbf{v}
$$

and as a result

$$
\Lambda(s)=\boldsymbol{\alpha}^{\prime}\left[\begin{array}{c}
\beta_{1} \\
\beta_{2} \\
\vdots \\
\beta_{N}
\end{array}\right]=\left[\begin{array}{llll}
\alpha^{0} & \alpha^{1} & \cdots & \alpha^{N-1}
\end{array}\right]\left[\begin{array}{c}
\beta_{1} \\
\beta_{2} \\
\vdots \\
\beta_{N}
\end{array}\right]
$$

where

$$
\beta_{i}=\frac{1}{\xi}\left(\rho^{N-i} \phi_{i}(s)+\sum_{j=i+1}^{N} \rho^{N-j}\left[\prod_{m=i}^{j-1}\left(1-\alpha^{m}\right) \prod_{m=i}^{j} \phi_{m}(s)\right]\right) .
$$

Thus

$$
\Lambda(s)=\frac{1}{\xi} \sum_{i=1}^{N} \alpha^{i-1}\left(\rho^{N-i} \phi_{i}(s)+\sum_{j=i+1}^{N} \rho^{N-j}\left[\prod_{m=i}^{j-1}\left(1-\alpha^{m}\right) \prod_{m=i}^{j} \phi_{m}(s)\right]\right)
$$

and total factor productivity is $\operatorname{TFP}(s)=A(\phi(s)) \Lambda(\phi(s))^{-1}$ where $A(\phi(s))$ satisfies (108) with scalar coefficients $a_{i}$ defined in (109). QED.

Proof of Lemma 1. (i) In this limit, $a_{i} \rightarrow 1$ and the labor wedge in (110) approaches

$$
\Lambda(s)=\prod_{i=1}^{N} \phi_{i}(s) \text { and } \operatorname{TFP}(s)=\frac{\prod_{i=1}^{N} \phi_{i}(s)}{\Lambda(s)}=1 .
$$


Therefore

$$
\chi_{i}(s)=\frac{d \log \Lambda(s)}{d \log \phi_{i}}=1, \quad \forall i \in I, s \in S .
$$

(ii) Let $\alpha=1$. Substituting this value into (110), we have that the labor wedge is given by

$$
\Lambda(s)=\sum_{i=1}^{N} v_{i} \phi_{i}(s)=\frac{1}{\xi} \sum_{i=1}^{N} \phi_{i}(s) \rho^{N-i}
$$

Taking the derivative of the labor wedge with respect to the distortion in sector $i$ gives us

$$
\chi_{i}(s)=\frac{d \log \Lambda(s)}{d \log \phi_{i}}=\frac{\phi_{i}(s)}{\Lambda(s)} \frac{d \Lambda(s)}{d \phi_{i}}
$$

Therefore

$$
\chi_{i}(s)=\frac{v_{i} \phi_{i}(s)}{\Lambda(s)}, \quad \forall i \in I, s \in S
$$

\section{QED.}

Proof of Proposition 6. Part (i). We let $\alpha \rightarrow 0$ for fixed $\rho$. The labor wedge in (110) approaches

$$
\Lambda(s) \rightarrow \frac{1}{\xi}\left(\sum_{i=1}^{N} \rho^{N-i}\left[\prod_{m=1}^{i} \phi_{m}(s)\right]\right)
$$

Moreover the coefficients in (30) approach

$$
a_{i} \rightarrow \frac{1}{\xi}\left(\sum_{j=i}^{N} \rho^{N-j}\right)
$$

Note that in the case of sector $1, a_{1}=1$.

Computing the elasticity of (112) with respect to the distortion in sector $j$ we get

$$
\chi_{j}(s)=\frac{d \log \Lambda(s)}{d \log \phi_{j}}=\frac{\phi_{j}(s)}{\Lambda(s)} \frac{d \Lambda(s)}{d \phi_{j}}=\frac{1}{\Lambda(s)} \frac{1}{\xi}\left(\sum_{i=j}^{N} \rho^{N-i}\left[\prod_{m=1}^{i} \phi_{m}(s)\right]\right)
$$

Therefore

$$
\chi_{j}(s)=\frac{\sum_{i=j}^{N} \rho^{N-i}\left[\prod_{m=1}^{i} \phi_{m}(s)\right]}{\sum_{i=1}^{N} \rho^{N-i}\left[\prod_{m=1}^{i} \phi_{m}(s)\right]}
$$

and again note that in the case of sector $1, \chi_{1}(s)=1$ for all $s$. 
The difference in elasticities between sector $j$ and sector $j+1$ is given by

$\chi_{j}(s)-\chi_{j+1}(s)=\frac{\sum_{i=j}^{N} \rho^{N-i}\left[\prod_{m=1}^{i} \phi_{m}(s)\right]-\sum_{i=j+1}^{N} \rho^{N-i}\left[\prod_{m=1}^{i} \phi_{m}(s)\right]}{\sum_{i=1}^{N} \rho^{N-i}\left[\prod_{m=1}^{i} \phi_{m}(s)\right]}=\frac{\rho^{N-j}\left[\prod_{m=1}^{j} \phi_{m}(s)\right]}{\sum_{i=1}^{N} \rho^{N-i}\left[\prod_{m=1}^{i} \phi_{m}(s)\right]}$

Thus, for any state $s$,

$$
\chi_{j}(s)-\chi_{j+1}(s)=\frac{\rho^{N-j}\left[\prod_{m=1}^{j} \phi_{m}(s)\right]}{\sum_{i=1}^{N} \rho^{N-i}\left[\prod_{m=1}^{i} \phi_{m}(s)\right]} \in(0,1),
$$

and is therefore strictly positive. As a result, for any state $s$, the following strict ordering holds:

$$
\chi_{1}(s)>\chi_{2}(s)>\cdots>\chi_{N}(s)
$$

where $\chi_{1}(s)=1$.

Finally we consider TFP in this limit. Note that we may rewrite the labor wedge in (112) as

$$
\Lambda(s)=\frac{1}{\xi} \phi_{1}(s)\left(\rho^{N-1}+\sum_{i=2}^{N} \rho^{N-i}\left[\prod_{m=2}^{i} \phi_{m}(s)\right]\right)
$$

by factoring out $\phi_{1}(s)$. TFP may therefore be written as

$$
\operatorname{TFP}(s)=\frac{\prod_{i=1}^{N} \phi_{i}(s)^{a_{i}}}{\frac{1}{\xi} \phi_{1}(s)\left(\rho^{N-1}+\sum_{i=2}^{N} \rho^{N-i}\left[\prod_{m=2}^{i} \phi_{m}(s)\right]\right)}
$$

where the coefficients $a_{i}$ are given by (113). Recall that for sector $1, a_{1}=1$. Canceling terms, TFP thereby reduces to

$$
\operatorname{TFP}(s)=\frac{\prod_{i=2}^{N} \phi_{i}(s)^{a_{i}}}{\frac{1}{\xi}\left(\rho^{N-1}+\sum_{i=2}^{N} \rho^{N-i}\left[\prod_{m=2}^{i} \phi_{m}(s)\right]\right)},
$$

and is independent of $\phi_{1}(s)$ but not independent of other sectors' distortions.

Part (ii). We let $\rho \rightarrow 0$ for fixed $\alpha$. Then $\xi \rightarrow 1$ and the labor wedge in (110) approaches

$$
\Lambda(s) \rightarrow \alpha^{N-1} \phi_{N}(s)+\phi_{N}(s) \sum_{i=1}^{N-1} \alpha^{i-1}\left(\prod_{m=i}^{N-1}\left(1-\alpha^{m}\right) \phi_{m}(s)\right) .
$$

Moreover the coefficients in (30) approach

$$
a_{i} \rightarrow \prod_{m=i}^{N-1}\left(1-\alpha^{m}\right) \text { for } i=1, N-1
$$


and in the case of sector $N, a_{N}=1$.

Computing the elasticity of (114) with respect to the distortion in sector 1 we get

$$
\chi_{1}(s)=\frac{\phi_{N}(s)}{\Lambda(s)}\left(\prod_{m=1}^{N-1}\left(1-\alpha^{m}\right) \phi_{m}(s)\right)
$$

Computing the elasticity of (114) with respect to the distortion in sector 2, we get

$$
\chi_{2}(s)=\frac{\phi_{N}(s)}{\Lambda(s)}\left(\prod_{m=1}^{N-1}\left(1-\alpha^{m}\right) \phi_{m}(s)+\alpha \prod_{m=2}^{N-1}\left(1-\alpha^{m}\right) \phi_{m}(s)\right)
$$

One may verify that for any state $s$ and for all sectors $j<N$, the following holds:

$$
\chi_{j}(s)-\chi_{j-1}(s)=\frac{\phi_{N}(s)}{\Lambda(s)}\left(\alpha^{j-1} \prod_{m=j}^{N-1}\left(1-\alpha^{m}\right) \phi_{m}(s)\right) \in(0,1)
$$

and in the case of $j=N$,

$$
\chi_{N}(s)-\chi_{N-1}(s)=\frac{\phi_{N}(s)}{\Lambda(s)} \alpha^{N-1} \in(0,1)
$$

and is therefore strictly positive.

Finally, computing the elasticity of (114) with respect to the distortion in sector $N$ we get

$$
\chi_{N}(s)=\frac{d \log \Lambda(s)}{d \log \phi_{N}}=\frac{\phi_{N}(s)}{\Lambda(s)}\left(\alpha^{N-1}+\sum_{i=1}^{N-1} \alpha^{i-1}\left(\prod_{m=i}^{N-1}\left(1-\alpha^{m}\right) \phi_{m}(s)\right)\right)=1
$$

As a result, for any state $s$, the following strict ordering holds:

$$
\chi_{1}(s)<\chi_{2}(s)<\cdots<\chi_{N}(s)
$$

where $\chi_{N}(s)=1$.

Finally we consider TFP in this limit. Note that we may rewrite the labor wedge in (114) as

$$
\Lambda(s)=\phi_{N}(s)\left[\alpha^{N-1}+\sum_{i=1}^{N-1} \alpha^{i-1}\left(\prod_{m=i}^{N-1}\left(1-\alpha^{m}\right) \phi_{m}(s)\right)\right]
$$

by factoring out $\phi_{N}(s)$. TFP may therefore be written as

$$
\operatorname{TFP}(s)=\frac{\prod_{i=1}^{N} \phi_{i}(s)^{a_{i}}}{\phi_{N}(s)\left[\alpha^{N-1}+\sum_{i=1}^{N-1} \alpha^{i-1}\left(\prod_{m=i}^{N-1}\left(1-\alpha^{m}\right) \phi_{m}(s)\right)\right]}
$$




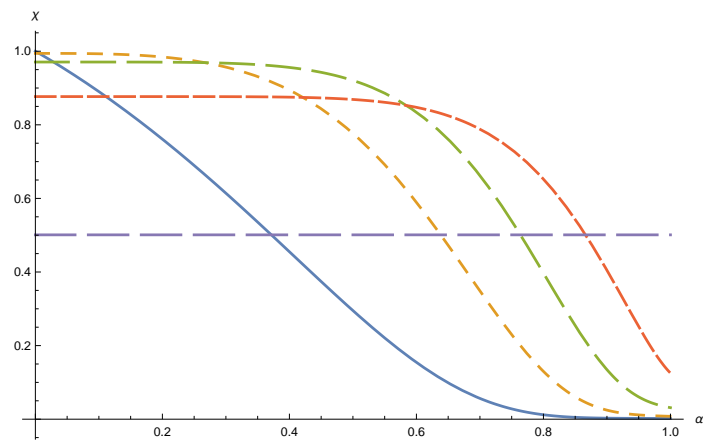

Figure 11: $\chi_{i}^{*}$ as a function of $\alpha$, with $\rho=.5$

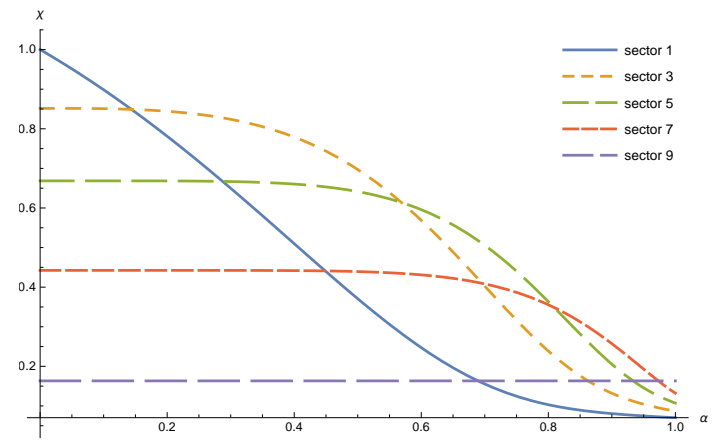

Figure 12: $\chi_{i}^{*}$ as a function of $\alpha$, with $\rho=.9$

where the coefficients $a_{i}$ are given by (115). Recall that for sector $N, a_{N}=1$. Canceling terms, TFP thereby reduces to

$$
\operatorname{TFP}(s)=\frac{\prod_{i=1}^{N-1} \phi_{i}(s)^{a_{i}}}{\alpha^{N-1}+\sum_{i=1}^{N-1} \alpha^{i-1}\left(\prod_{m=i}^{N-1}\left(1-\alpha^{m}\right) \phi_{m}(s)\right)},
$$

and is independent of $\phi_{N}(s)$ but not independent of other sectors' distortions. QED.

\section{Comparative statics for intermediate values of $\alpha$ and $\rho$.}

We now consider intermediate values of $(\alpha, \rho) \in(0,1]^{2}$ and examine the comparative statics of labor wedge influence as we move continuously within the extremes.

In three of the four limits of the hybrid economy presented in the main text, we were able to provide a strict ordering across sectors in terms of their labor wedge influence. These orderings were both monotonic and independent of the aggregate state. Away from these limits, however, this independence property does not necessarily hold: the ordering of labor wedge influence is generically a function of the aggregate state $s$. Thus, in order to maintain tractability, we study the comparative statics of labor wedge influence at efficiency. Recall that at efficiency, $\chi_{i}^{*}=a_{i}$ where $a_{i}$ are scalar coefficients that depend on the parameters $\alpha$ and $\rho$ alone. Specifically we consider an economy with $N=9$ sectors and compute for each sector $\chi_{i}^{*}$ as a function of $(\alpha, \rho)$ according to (30). We then examine how $\chi_{i}^{*}$ changes as we vary $\alpha$ and $\rho$. We present the comparative statics of $\chi_{i}^{*}$ with respect to $\alpha$ for all odd numbered sectors in Figures 11 and 12 holding $\rho$ fixed at two different values. Similarly we present the comparative statics of $\chi_{i}^{*}$ with respect to $\rho$ for all odd numbered sectors in Figures 13 and 14 holding $\alpha$ fixed at two different values.

Consider the comparative statics presented in Figures 11 and 12. We already know that for fixed $\rho$,in the limit in which $\alpha \rightarrow 0$, sectors are monotonically decreasing in labor wedge influence: $1=\chi_{1}^{*}>\cdots>\chi_{N}^{*}$. This strict ordering holds in the far left of these figures. We furthermore 


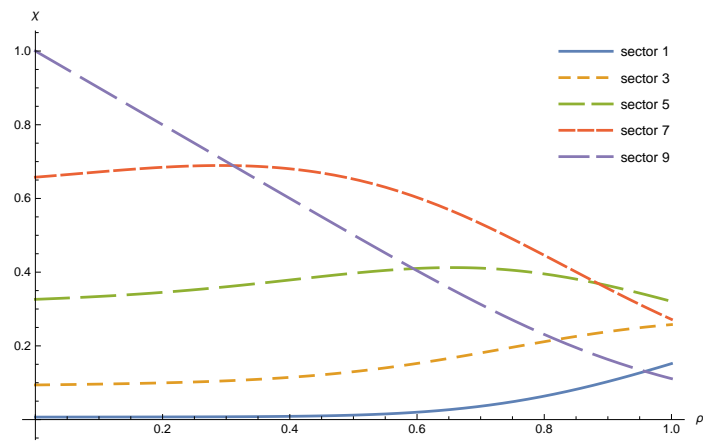

Figure 13: $\chi_{i}^{*}$ as a function of $\rho$, with $\alpha=.5$

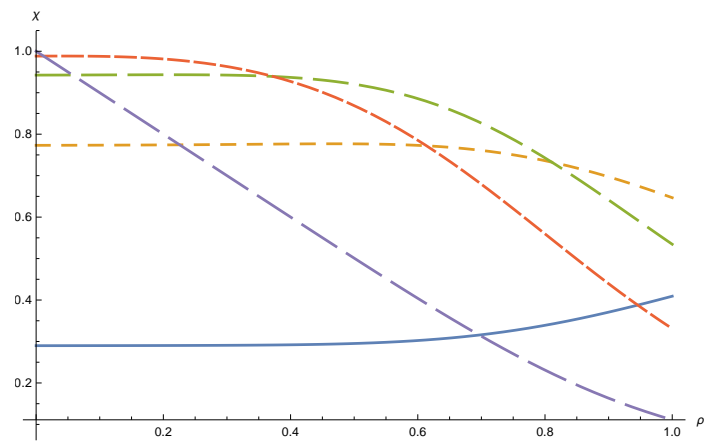

Figure 14: $\chi_{i}^{*}$ as a function of $\rho$, with $\alpha=.9$

know at the other extreme in which $\alpha \rightarrow 1$, sectors are monotonically increasing in labor wedge influence: $\chi_{1}^{*}<\cdots<\chi_{N}^{*}$. This strict ordering holds in the far right of these figures. Thus, as $\alpha$ moves continuously from zero to one, i.e. as the labor share of output grows and the intermediate good share falls, we see that sector 1 is the first to start losing labor wedge influence, then sector 2 , then sector 3 , and so on. As $\alpha$ approaches one, the ordering across sectors completely reverses.

Moreover, note that for intermediate values of $\alpha$, the ordering across sectors is non-monotonic. Fixing $\rho$, for any sector $i$ there exists a non-empty interval of $\alpha$, such that sector $i$ has the greatest labor wedge influence for $\alpha$ in that interval. However, as $\alpha$ increases and the strength of inputoutput linkages falls, upstream sectors become less important relative to downstream sectors in terms of their aggregate labor wedge influence.

Consider now the comparative statics presented in Figures 13 and 14. Again we already know that for fixed $\alpha$, in the limit in which $\rho \rightarrow 0$, sectors are monotonically increasing in labor wedge influence: $\chi_{1}^{*}<\cdots<\chi_{N}^{*}=1$. This strict ordering holds in the far left of these figures. However, we have no such monotonicity at the other extreme. Therefore, the ordering changes as one moves $\rho$ continuously from zero to one, but we do not have the property that the ordering completely reverses as $\rho$ approaches one.

\section{Appendix C: Supporting Quantitative Analysis \& Figures}

\section{Heterogeneity in Labor, Intermediate Good, and Consumption Shares.}

In Figures 15 and 16 we report the top 20 industries in our sample with the greatest labor shares of expenditure and the greatest intermediate good shares of expenditure, $\alpha_{i}$ and $1-\alpha_{i}$, respectively, in 2007. Industries with the greatest labor shares are government sectors, services (computer systems design, legal services), nursing and care facilities, education, and retail industries (e.g. motor vehicle dealers). On the other hand, the industries with the greatest intermediate good shares, equivalently the lowest labor shares, are generally primary industries such as oil and gas, farming, 


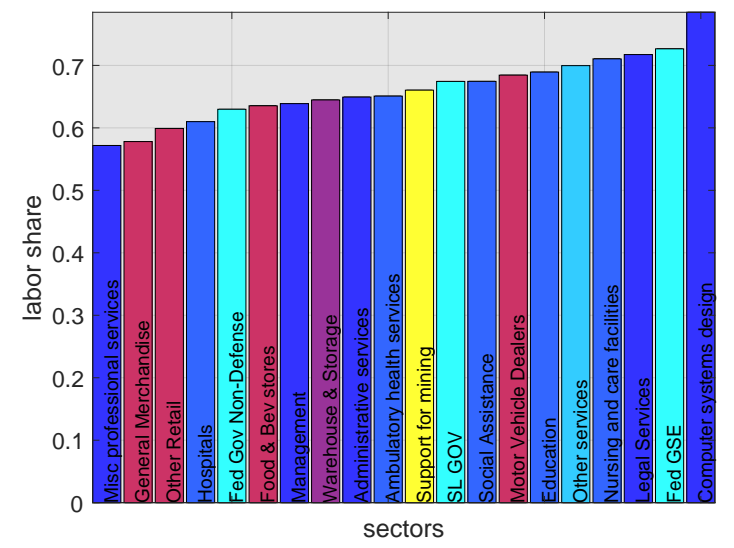

Figure 15: Top 20 Labor Shares

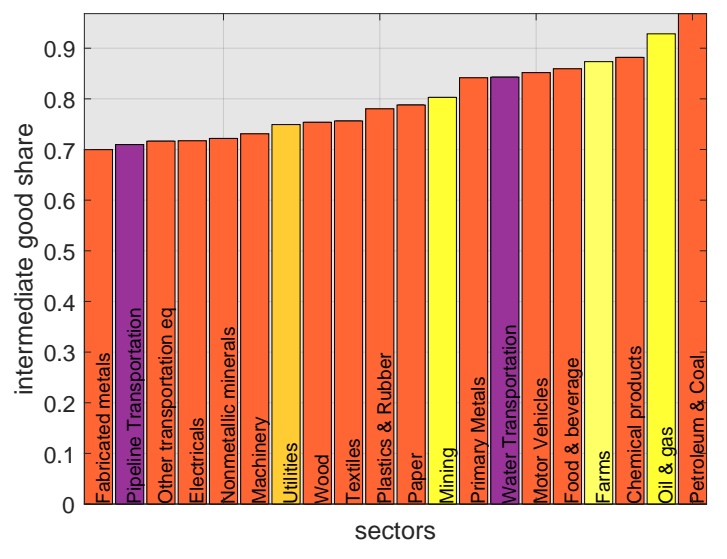

Figure 16: Top 20 Intermediate Good Shares

mining, as well as manufacturing industries such as petroleum \& coal, chemical products, motor vehicle manufacturing, metals, and textiles.

In Figure 17 we report the top 20 industries with the greatest final use shares, $v_{i}$, in 2007 . The sectors with the largest shares in final uses are government sectors, construction, wholesale, as well as an array of services, such as healthcare, ambulances, professional services, and education.

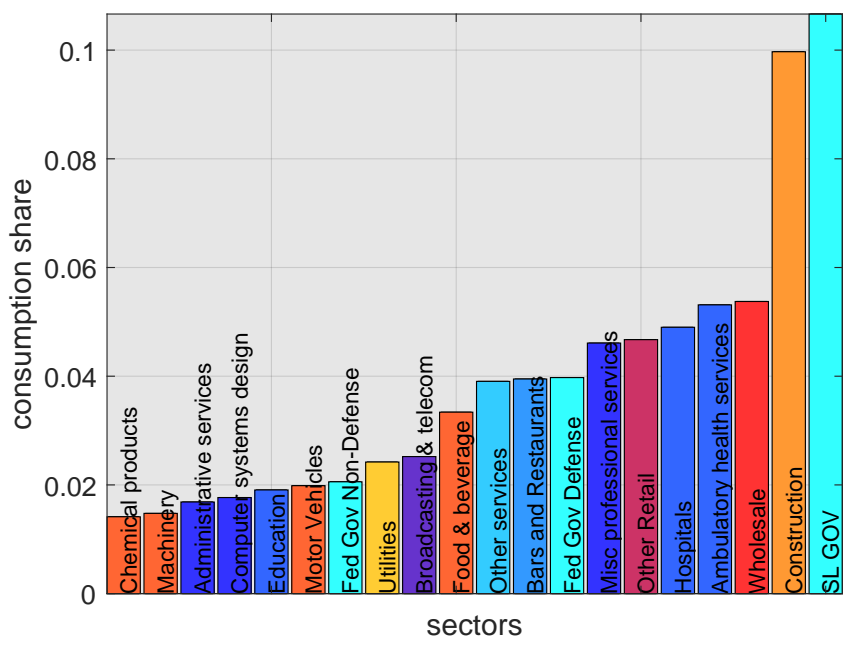

Figure 17: Top 20 Consumption Shares

\section{Time-Series Statistics for Constructed Quantities.}

As described in the main text, we construct sectoral measures of output, hours, and inputs by merging the BLS and BEA data and backing out quantities. These constructed quantities are never used as inputs to the model; we construct them simply for diagnostic purposes at the sectoral level. 


\begin{tabular}{|l|c|c|c|c|}
\hline & All Sectors & \multicolumn{3}{|c|}{ Manufacturing Sectors } \\
\hline & & All & Durable & Non-Durable \\
\hline \hline $\operatorname{std}(\Delta \log y)$ & 0.055 & 0.075 & 0.087 & 0.059 \\
\hline $\operatorname{std}(\Delta \log l)$ & 0.041 & 0.048 & 0.056 & 0.038 \\
\hline $\operatorname{std}(\Delta \log x)$ & 0.128 & 0.107 & 0.110 & 0.104 \\
\hline $\operatorname{corr}(\Delta \log y, \Delta \log l)$ & 0.529 & 0.648 & 0.749 & 0.509 \\
\hline $\operatorname{corr}(\Delta \log y, \Delta \log x)$ & 0.688 & 0.887 & 0.943 & 0.810 \\
\hline
\end{tabular}

Table 3: Sectoral Quantities Time Series Statistics

Table 3 provides within-sector time-series statistics for our constructed series of sectoral output, hours, and inputs. Within each sector we calculate the standard deviation over time of $\Delta \log y_{i t}$, $\Delta \log \ell_{i t}$ and $\Delta \log x_{i t}$; we report the cross-sectional means of these standard deviations in the first three rows of Table 3. The mean standard deviation for log changes in output is around 5.5\%. Hours are slightly less volatile than output, while inputs are more volatile. Within each sector we also calculate the time-series correlation between log changes in output with those of hours and inputs within each sector; we report the cross-sectional means of these correlations in the last two rows of Table 3. Movements in sectoral output are strongly positively correlated with movements in both hours and inputs - 0.53 and 0.69 respectively.

The first column of Table 3 reports these statistics for all sectors in our sample. The subsequent columns report the same within-sector time-series statistics for the sub-sample of manufacturing industries; within this sub-sample, we further split the sample between durable and non-durable manufacturers. Manufacturing sectors, particularly durable manufacturers, are on average slightly more volatile than all sectors and moreover display relatively higher correlations between output and inputs.

\section{Time-Series Properties of Distortions for Individual Industries.}

As described in the main text, within the typical sector there is positive co-movement between log changes in GZ distortions and output; on average this correlation is $31 \%$. We now take a closer look at this relationship at the individual industry level. In Figure 18 we plot $\Delta \log \phi_{i t}$ and $\Delta \log y_{i t}$, that is, the sector's log change in distortions and output, for eight specific industries. These industries are: utilities, construction, durable manufacturing sectors (wood, fabricated metals, computers, electricals, and motor vehicles) and a non-durable manufacturing sector: plastics \& rubber products. It is clear from this figure that for these sectors, as with most other sectors, the measured GZ distortion co-moves positively with sectoral output.

Also as described in the main text, within the typical sector there is weak positive co-movement between log changes in GZ distortions and in expenditure shares; on average this correlation is 

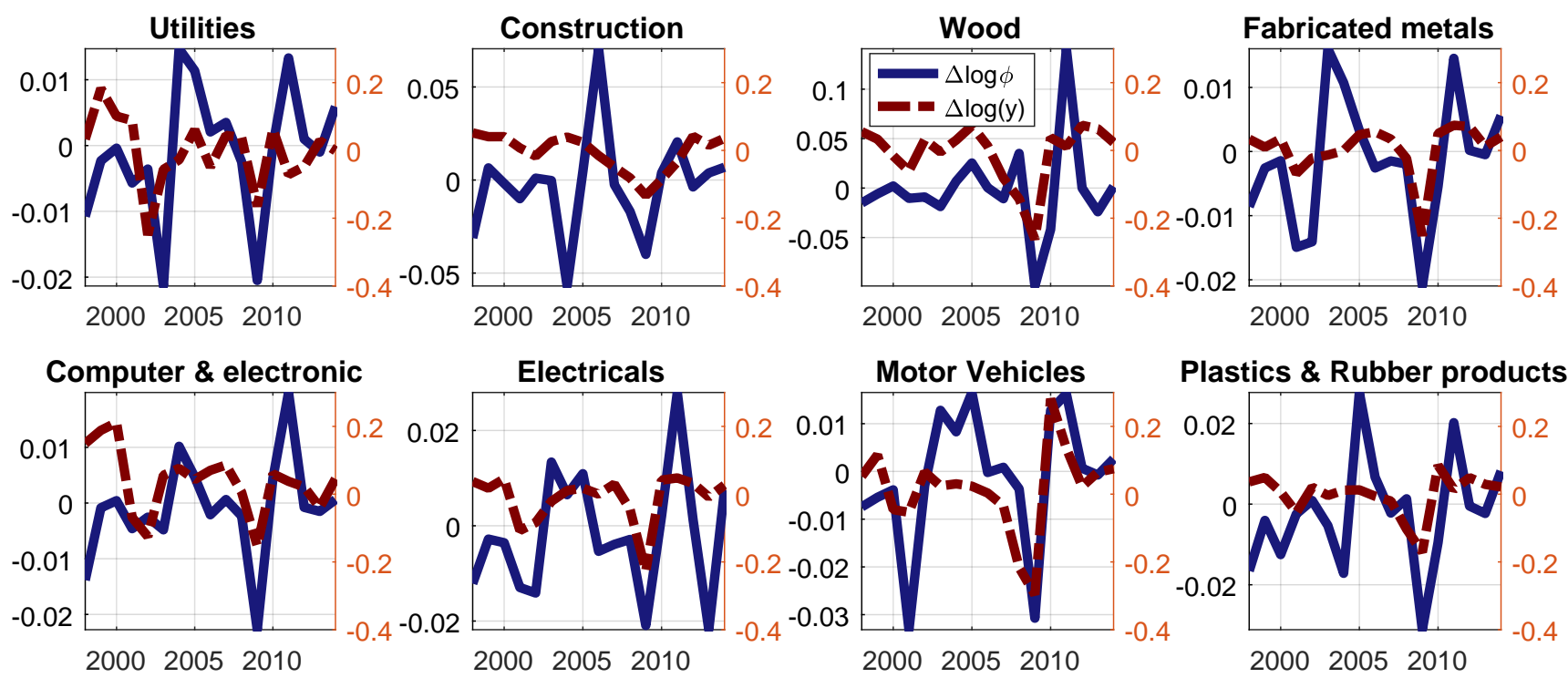

Figure 18: Time series for $\Delta \log \phi_{i t}$ (left axes) and $\Delta \log y_{i t}$ (right axes) within 8 specific industries.

19.5\%. We again take a closer look at this relationship at the individual industry level. In Figure 19 we plot $\Delta \log \phi_{i t}$ and $\Delta \log \varepsilon_{i t}$, that is, the sector's log changes in distortions and expenditure shares, for the same eight industries highlighted in Figure 18. While these two measures are weakly correlated at the industry level, we do generally observe a fall in both measures across industries from 2007 to 2009.

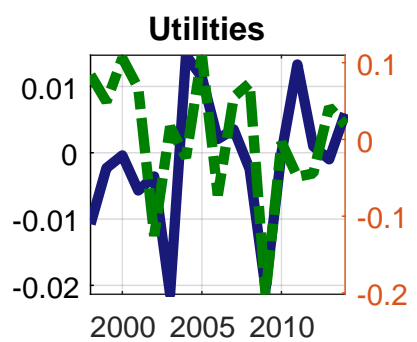

Computer \& electronic

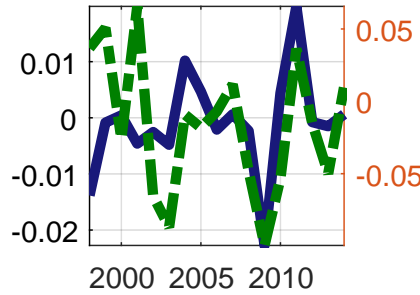

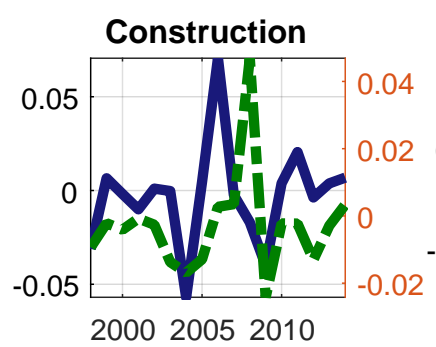

Electricals

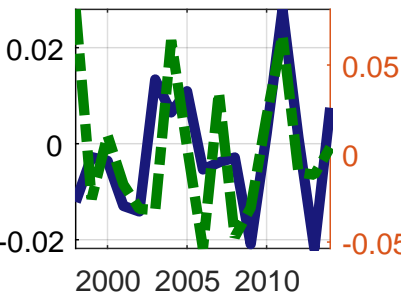

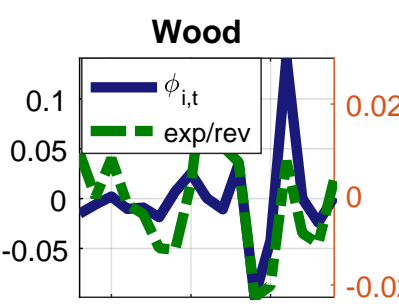

200020052010
Fabricated metals

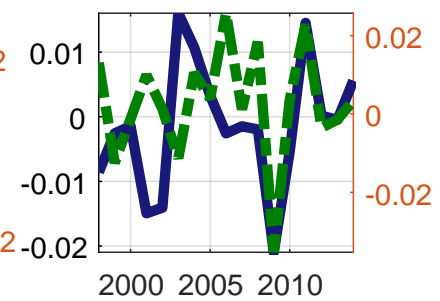

Plastics \& Rubber products

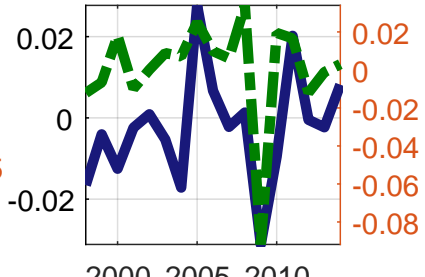

Figure 19: Time series for $\Delta \log \phi_{i t}$ (left axes) and $\Delta \log \varepsilon_{i t}$ (right axes) within 8 specific industries. 


\section{Relation to the Network Multiplier in Jones (2011) and Jones (2013).}

Consider the following simple one-sector, round-about economy. The aggregate production function is given by $Y(s)=z(s) X(s)^{1-\alpha} L(s)^{\alpha}$ where $Y$ is gross output, $X$ denotes intermediate goods, $L$ is labor, and $\alpha$ is the labor share. We drop the $i$ subscript as there is only one sector and we let $z(s)$ denote the productivity in this single sector. The aggregate resource constraint is given by $C(s)+X(s)=Y(s)$, where $C(s)$ is aggregate consumption, i.e. GDP. This round-about economy serves as a common backbone of certain models used to analyze the intermediate good multiplier effect, see e.g. Basu (1995), Jones (2011), and Jones (2013). We use this simple economy to illustrate and compare our results to some of those found in the literature.

The Efficient Economy. Consider first the competitive equilibrium in which there are no distortions. For now we assume that labor supply is fixed. Given that the equilibrium is efficient, we may solve for it simply by solving the planner's problem. The planner maximizes consumption subject to the resource constraint. Due to Cobb-Douglas technology, $X(s)=(1-\alpha) Y(s)$. Combining this with the resource constraint implies $C(s)=\alpha Y(s)$. Substituting these into the production function and solving for GDP per worker yields

$$
\operatorname{TFP}(s) \equiv \frac{C(s)}{L(s)}=\alpha(1-\alpha)^{\frac{1-\alpha}{\alpha}} z(s)^{\frac{1}{\alpha}}
$$

Jones (2011) and Jones (2013) use this equation to make the argument that the network multiplier in this economy is $1 / \alpha$. Specifically, this is the TFP network multiplier of productivity:

$$
\frac{d \log T F P(s)}{d \log z(s)}=\frac{1}{\alpha}
$$

Jones (2011) and Jones (2013) show how distortions at the micro level create input misallocation, thereby leading to a fall in TFP.

Jones (2013) notes that, "small departures from the optimal allocation of labor have tiny effects on TFP (an application of the envelope theorem), but significant misallocation can have very large effects... In the presence of significant misallocation, a small improvement in the allocation of resources will have a large impact on TFP." Therefore, in order for micro distortions to have a large effect on TFP, the economy must be away from efficiency; we believe inefficient economies are the type of economies Jones (2013) has in mind.

The Distorted Economy. Now consider the same economy but allow for endogenous labor supply and for a wedge that distorts the single sector's representative firm's decision. This firm maximizes profits, $\phi(s) P(s) Y(s)-P(s) X(s)-L(s)$ where $\phi(s) \equiv 1-\tau(s)$ may be interpreted as a distortion resulting from a revenue tax; we assume the tax proceeds are rebated to the household, so that the 
resource constraint continues to satisfy $C(s)+X(s)=Y(s)$. One can show that in equilibrium, a labor wedge arises and is given by

$$
\Lambda(s)=\frac{\alpha \phi(s)}{1-(1-\alpha) \phi(s)}
$$

To a first-order log approximation of this labor wedge around efficiency, one obtains

$$
\left.\frac{d \log \Lambda(s)}{d \log \phi(s)}\right|_{\phi=1} \approx \frac{1}{\alpha}
$$

Therefore, the labor wedge network multiplier of distortions is $1 / \alpha$ and thereby equal to the TFP network multiplier in (117).

Connection between the two different multipliers. As already stressed in our theoretical section, despite being conceptually different, there is a close connection between the two multipliers presented in (117) and (119). Theorem 2 informs us that up to a first order approximation around efficiency, the two should coincide:

$$
\left.\frac{d \log \Lambda(s)}{d \log \phi(s)}\right|_{\phi=1} \approx \frac{d \log T F P(s)}{d \log z(s)} .
$$

Next, from Hulten's theorem, or more generally from the envelope condition of the planner's problem, the multiplier presented Jones $(2011,2013)$ should be equal to the the ratio of gross output over GDP. As a result:

$$
\left.\frac{d \log \Lambda(s)}{d \log \phi(s)}\right|_{\phi=1} \approx \frac{d \log T F P(s)}{d \log z(s)}=\frac{Y(s)}{C(s)}
$$

thereby illustrating our result in Corollary 2.

Finally, equation (120) holds in any general input-output economy of the class studied in this paper. However, the final step is the structural interpretation of this equation. In this simple roundabout economy, the efficient allocation has that $C(s)=\alpha Y(s)$. Therefore, gross sales over GDP is given by $Y(s) / C(s)=1 / \alpha$; combining this with equation (120) gives us the desired network multiplier results presented in (117) and (119)

However, note that this last step - the multiplier's structural interpretation - is specific to this particular economy. More generally, as we have shown above in equation (36), the following must hold:

$$
\left.\frac{d \log \Lambda(s)}{d \log \phi(s)}\right|_{\phi=1} \approx \frac{d \log T F P(s)}{d \log z(s)}=\mathbf{v}^{\prime} \mathbf{B} \mathbf{1}=\mathbf{v}^{\prime}\left[\mathbb{I}_{N}-\operatorname{diag}(\mathbf{1}-\boldsymbol{\alpha}) \mathbf{G}\right]^{-1} \mathbf{1}
$$

where $\mathbf{B}$ is the Leontief inverse matrix. The round-about economy is a special case of the general economy in which there is only one sector, so that $\mathbf{v}$ and $\mathbf{G}$ are both scalars equal to 1 . Thus, applying equation $(121)$ we have that $\mathbf{v}^{\prime} \mathbf{B} \mathbf{1}=(1-(1-\alpha))^{-1}=1 / \alpha$. 
To conclude, the TFP network multiplier of productivity presented in (117) and the labor wedge network multiplier of distortions in (119) are conceptually different objects. However, our theoretical results prove that there is a tight connection between the two. Up to a first-order approximation around efficiency these objects are equal. Moreover, a sufficient statistic for these multipliers is the ratio of gross sales to GDP. Away from efficiency, however, this equality need not hold. Finally, while the network multiplier in the round-about simple economy is structurally equal to $1 / \alpha$, more generally the network multiplier is equal to $\mathbf{v}^{\prime} \mathbf{B} \mathbf{1}$ and therefore need not coincide with $1 / \alpha$.

Proofs for the Network Multipliers presented above. The Efficient Economy. We solve the planner's problem. The planner maximizes consumption subject to the resource constraint. Due to Cobb-Douglas technology, $X(s)=(1-\alpha) Y(s)$. Substituting this into the production function and solving for gross output per worker gives us

$$
\frac{Y(s)}{L(s)}=(1-\alpha)^{\frac{1-\alpha}{\alpha}} z(s)^{\frac{1}{\alpha}}
$$

Moreover, from the resource constraint we have $C(s)=\alpha Y(s)$. Therefore, TFP, or equivalently GDP per worker, may be expressed as in (116).

The Distorted Economy. We allow for endogenous labor supply and for a wedge that distorts the single sector's representative firm's decision. This firm maximizes profits

$$
\Pi(s)=\phi(s) P(s) Y(s)-P(s) X(s)-L(s)
$$

subject to the production function $Y(s)=z(s) X(s)^{1-\alpha} L(s)^{\alpha}$. This yields FOCs

$$
\begin{gathered}
\phi(s)(1-\alpha) Y(s)=X(s) \\
\phi(s) \alpha P(s) Y(s)=L(s)
\end{gathered}
$$

Substituting the firm's optimality condition for intermediate goods (122) into the resource constraint and solving for gross output yields:

$$
Y(s)=\frac{1}{1-\phi(s)(1-\alpha)} C(s) .
$$

On the household's side, we have the following optimality condition:

$$
\frac{V^{\prime}(L(s))}{U^{\prime}(C(s))}=\frac{1}{P(s)}
$$


Combining this with the firm's optimality condition for labor (123) gives us

$$
\frac{V^{\prime}(L(s))}{U^{\prime}(C(s))}=\phi(s) \alpha \frac{Y(s)}{L(s)}
$$

Finally, replacing gross output in the above expression with (124), yields the following equilibrium condition.

$$
\frac{V^{\prime}(L(s))}{U^{\prime}(C(s))}=\frac{\phi(s) \alpha}{1-\phi(s)(1-\alpha)} \frac{C(s)}{L(s)}
$$

Therefore the aggregate labor wedge is given by

$$
\Lambda(s)=\frac{\alpha \phi(s)}{1-(1-\alpha) \phi(s)}
$$

as in (118). Taking the derivative of this expression with respect to the distortion $\phi(s)$ yields.

$$
\frac{d \Lambda(s)}{d \phi(s)}=\frac{\alpha-\alpha(1-\alpha) \phi(s)+\alpha(1-\alpha) \phi(s)}{(1-(1-\alpha) \phi(s))^{2}}=\frac{\alpha}{(1-(1-\alpha) \phi(s))^{2}}
$$

Finally, evaluating this at the efficient benchmark gives us

$$
\left.\frac{d \Lambda(s)}{d \phi(s)}\right|_{\phi(s)=1}=\frac{\alpha}{(1-(1-\alpha))^{2}}=\frac{1}{\alpha}
$$

as in (119). QED.

\section{Appendix D: Description of the Data}

Below we describe each data source used in our quantitative illustration.

\section{BEA Commodity Use Data.}

We work with the Use of Commodities by Industries After Redefinitions (Uses AR) provided by the Bureau of Economic Analyis (BEA). The USES AR tables are organized in the following way: each row corresponds to a sectoral commodity and each column to an industry or component of aggregate demand (consumption, investment, etc). An entry in the table reports the expenditures on the commodity of the corresponding row by the industry of the corresponding column (in billions of US dollars). We use this table to construct the input-output mattix of the US corresponding to our model.

The NIPA categories are based on attributes of the goods and services (commodities) produced by an industry. The Uses AR table is constructed from another table called the the Uses of Commodities by Industries Before Redefinitions (Uses BR). The data in the Uses BR table is 
constructed from firm level data. However, several firms produce goods that fall into different product categories. The Uses AR thereby reorganizes the data in the Uses BR by reassigning a portion of commodity uses of a given industry to another industry when the firms in the former also produce the product of the latter. ${ }^{41}$

First, we make adjustments so that there are the same number of input sectors as output sectors in order maintain an $N \times N$ IO table. Not all commodities correspond to industries at the five digit level. To construct an $N \times N$ IO table, we assign the uses of the Secondary smelting and alloying of aluminum (331314) sector to merge Alumina refining and primary aluminum production (33131a). We do the same for the Federal electric utilities (S00101) sector which is allocated to Federal general government (nondefense) (S00600) sector and for State and local government passenger transit (S00201) and State and local government electric utilities (S00202) which are both allocated to State and local general government (S00203). Finally, the input-output tables also report entries for used goods and scrap. We reassign these rows across all other rows according to their proportion.

Treatment of Finance, Insurance, and Real-Estate Financing (FIRE). Second, we treat finance, insurance, real estate, rental and leasing (FIRE) sectors as special sectors. The Uses AR tables report transfers from each sector to each of the the FIRE industries. However, we do not want to treat payments to these industries as payments for intermediate inputs, the reason being that we interpret the production function as related to the physical of production: if a given sector purchases a given volume of production inputs, production would be the same. In contrast, interest rates, insurance premia, rental rates and leases we do not consider to be payments for intermediate inputs.

Therefore, we exclude all rows and columns corresponding to the Finance, Insurance, and RealEstate (FIRE) sectors and renormalize the Input-Output matrix. At the five digit level, these industries correspond to those found in Table 4.

Treatment of Imports and Exports. Third, our model is a closed economy while the Uses AR tables include imports and exports. In the data, several industries show a negative trade balance, which in principle means that certain intermediate inputs could be partially supplied by an external sector.

However, it turns out that for all industries, except for oil, domestic absorption exceeds any negative trade balance. Even at the five digit level, most inputs used in the US are produced within the US. In fact, most commodities in the US are both imported and produced domestically, and used as inputs and final goods. There are some commodities that are imported but, for all commodities

\footnotetext{
${ }^{41}$ For robustness we also check that our results hold if we use the USES Before Redefinitions table (USES BR).
} 


\begin{tabular}{|c|c|}
\hline NIPA & Sector Description \\
\hline $52 \mathrm{~A} 000$ & Monetary authorities and depository credit intermediation \\
\hline $522 \mathrm{~A} 00$ & Nondepository credit intermediation and related activities \\
\hline $523 \mathrm{~A} 00$ & Securities and commodity contracts intermediation and brokerage \\
\hline 523900 & Other financial investment activities \\
\hline 524100 & Insurance carriers \\
\hline 524200 & Funds, trusts, and other financial vehicles \\
\hline 525000 & Real estate \\
\hline 531000 & Automotive equipment rental and leasing \\
\hline 532100 & Consumer goods and general rental centers \\
\hline $532 \mathrm{~A} 00$ & Lessors of nonfinancial intangible assets \\
\hline 532400 & Commercial and industrial machinery and equipment rental and leasing \\
\hline 533000 &
\end{tabular}

Table 4: FIRE Sectors

that are produced in the US (except oil), final uses exceed imports. Thus, we treat all imports as if they are consumed by households - except for the case of oil, we ignore oil imports. We will think of households as purchasing US produced goods, exporting some of these and importing the rest for final consumption only. Thus, we treat all US production as final uses. When we consider the household's expenditure shares on domestic production, we exclude imports but include exports as part of the shares.

Treatment of Inventories. Finally, we correct for inventories. Part of the final uses of a given sector is inventory that will be used in final periods. Our model is static so there is no inventory accumulation. In practice, inventories can be stored as final goods, or as materials.

To account for inventories, we subtract inventories from total investment, and redistribute the dollar value of inventories supplied by a given sector by adding a share of total inventories to the uses by other sectors. The share of inventories we add to uses is proportional to the uses of each sector over total uses. For example, we raise the sales of cotton mills to the textile industry by adding the stock of cotton inventories times the proportion of cotton sold to the textile industry relative to all industries.

\section{BLS Hours Data.}

Sectoral data on hours are obtained from the Bureau of Labor Statistics at an annual frequency. In particular, we use data from the survey of Industry Productivity constructed from the program on Labor Productivity and Costs (LPC) measures for sectors of the U.S. economy. The data is produced by the Division of Industry Productivity Studies (IPS) in the Office of Productivity and Technology at the Bureau of Labor Statistics. The data is available at https://www.bls.gov/ 
lpc/home.htm. Details of the data are included in Chapter 11, "Industry Productivity Measures," of the BLS Handbook of Methods.

The Industry Productivity program produces annual measures of output per hour, unit labor costs, and related series for a select group of U.S. industries. The survey includes indices by industry for labor productivity (output per hour), output per person, real output, the implicit price deflator for output, labor hours, employment, labor compensation, and unit labor costs. In addition, the level data underlying the productivity and cost measures - including nominal value of production, labor compensation, total annual hours, and total annual employment. For most industries, the indexes predate the BEA's construction of IO tables annual tables in 1997. We specifically used the survey data available at: https://www.bls.gov/lpc/tables_by_sector_and_industry.htm.

The Industry pPoductivity survey classifies sectors according to the North American Industry Classification System (NAICS) as in the BEA data. The survey contains an index of sectoral hours. The BEA describes this series as "a hybrid measure of both hours worked a hours paid. [Labor] hours is a measure that represents the amount of time individuals contribute to produce output of domestic goods and services." We use this series as our sectoral labor series.

\section{Financial Distortions Data.}

For the Gilchrist and Zakrajšek (2012) (GZ) measure of the Excess Bond Premium (EBP), we obtain the data directly from the authors. The raw data is proprietary but the authors provide us with the sectoral measure of the index constructed in Gilchrist and Zakrajšek (2012); specifically it is the sectoral level version of equation (1) in that paper. The data was provided to us using the NAICS classification. Within each sector we have the cross-sectional mean, median and spread per outstanding bond; for our measure we use the cross-sectional mean. The typical year-sector observation has 60 bond observations although we cannot identify the number of issuers. Excluding the the Government subsectors, $98 \%$ of the sector-year observation contained an observation. We assigned a value of 0 to the GZ series if a sector didn't have observations.

As explained by the authors, the Excess Bond Premium is the residual from individual bond spreads obtained from Credit-Default Swap data. The spread is built relative to a constructed measure of risk-free bond rates. To measure a firm's probability of default at each point in time, the authors employ a "distance to default" model. We leave the rest of the details to that paper. 


\section{Appendix E: Proofs for Decreasing Returns to Scale model}

In this final appendix we solve for the equilibrium of the economy with decreasing returns to scale. As stated in equation (32), each firm has production function

$$
y_{i}(s)=z_{i}(s)\left(\ell_{i}(s)^{\alpha_{i}}\left(\prod_{j \in I} x_{i j}(s)^{g_{i j}}\right)^{1-\alpha_{i}}\right)^{\eta_{i}}
$$

where $\eta_{i} \in(0,1]$ denotes the sector's returns to scale parameter . We first state all the necessary results for the DRS economy; after that, we proceed with their proofs.

\section{DRS Economy Results}

We solve the case of decreasing returns to scale in a slightly different manner than in the CRS case. Rather than start with the allocation we first solve for equilibrium sales.

Lemma 5. Let $m_{i}(s)$ denote equilibrium sales in sector $i$ and let $\mathbf{m}(s) \equiv \operatorname{diag}(\mathbf{p}(s)) \mathbf{y}(s)$ denote the equilibrium vector of sales. Equilibrium sectoral sales are linear in aggregate labor

$$
\mathbf{m}(s)=\widetilde{\Omega}(\phi(s)) L(s)
$$

where $\widetilde{\Omega}: \mathbb{R}_{+}^{N} \rightarrow \mathbb{R}_{+}^{N}$ is an $N \times 1$ vector function given by

$$
\widetilde{\Omega}(\phi(s)) \equiv\left[\mathbb{I}_{N}-(\operatorname{diag}(\mathbf{1}-\alpha) \mathbf{G})^{\prime} \operatorname{diag}(\eta) \operatorname{diag}(\boldsymbol{\phi}(s))-\mathbf{v} \mathbf{1}^{\prime}\left(\mathbb{I}_{N}-\operatorname{diag}(\eta) \operatorname{diag}(\boldsymbol{\phi}(s))\right)\right]^{-1} \mathbf{v}
$$

Lemma 5 is the DRS analogue of Lemma 7 in the main text. Clearly moving to decreasing returns preserves the linearity of equilibrium sales in labor. However, the $\widetilde{\Omega}(s)$ vector is now adjusted for the decreasing returns. In the special case of CRS, $\widetilde{\Omega}(s)$ reduces to $\Omega(s)$. We next provide a lemma characterizing the vector of equilibrium prices in this economy.

Lemma 6. Given the vector of equilibrium sales, $\mathbf{m}(s)$, the vector of equilibrium sectoral prices is given by

$$
\log \mathbf{p}(s)=-\widetilde{\mathbf{B}}[\operatorname{diag}(\eta)(\log \mathbf{z}(s)+\log \phi(s)+\log \eta+\kappa)]+\widetilde{\mathbf{B}}\left[\mathbb{I}_{N}-\operatorname{diag}(\eta)\right] \log \mathbf{m}(s)
$$

where $\kappa=\left(\kappa_{1}, \ldots, \kappa_{N}\right)^{\prime}$ is an $N \times 1$ vector of constants and $\widetilde{\mathbf{B}}$ is the $N \times N$ matrix given by

$$
\widetilde{\mathbf{B}} \equiv\left[\mathbb{I}_{N}-\operatorname{diag}(\eta) \operatorname{diag}(\mathbf{1}-\alpha) \mathbf{G}\right]^{-1}
$$


We may call $\widetilde{\mathbf{B}}$ the DRS-adjusted Leontief inverse matrix. It is the same as the Leontief inverse matrix $\mathbf{B}$ in the CRS economy, except the network effects are slightly attenuated by the $\eta$ parameters.

Using these two results, we prove the following theorem on aggregation; it is the DRS analogue of Theorem 1 in the main text.

Theorem 3. There exists functions $\widetilde{T F P}: S \rightarrow \mathbb{R}_{+}$and $\widetilde{\Lambda}: S \rightarrow \mathbb{R}_{+}$such that the equilibrium of the production network economy with Cobb-Douglas technologies and preferences over goods aggregates to a representative firm, representative household economy with total factor productivity $\widetilde{T F P}(s)$ and an aggregate labor wedge $\widetilde{\Lambda}(s)$.

The equilibrium allocation in the representative firm, representative household economy is characterized by a decreasing returns-to-scale aggregate production function given by:

$$
C(s)=\widetilde{T F P}(s) L(s)^{\widetilde{\eta}},
$$

with returns to scale parameter

$$
\widetilde{\eta} \equiv 1-\mathbf{d}^{\prime} \mathbf{1} \in(0,1]
$$

where $\mathbf{d}^{\prime} \equiv \mathbf{v}^{\prime} \widetilde{\mathbf{B}}\left[\mathbb{I}_{N}-\operatorname{diag}(\eta)\right]$, and an equilibrium intratemporal condition given by

$$
\frac{V^{\prime}(L(s))}{U^{\prime}(C(s))}=\frac{\widetilde{\Lambda}(s)}{\widetilde{\eta}} M P_{L}(s)
$$

where the marginal product of aggregate labor is equal to $M P_{L}(s)=\widetilde{\eta} C(s) / L(s)$. The functions $\widetilde{\Lambda}(s)$ and $\widetilde{T F P}(s)$ are defined as follows.

(i) The aggregate labor wedge $\widetilde{\Lambda}: S \rightarrow \mathbb{R}_{+}$is given by

$$
\widetilde{\Lambda}(s) \equiv\left[1+\mathbf{1}^{\prime}\left(\mathbb{I}_{N}-\operatorname{diag}(\eta) \operatorname{diag}(\phi(s))\right) \widetilde{\Omega}(s)\right]^{-1} .
$$

where $\widetilde{\Omega}(s)$ is the $N \times 1$ vector defined in $(127)$.

(ii) Total factor productivity $\widetilde{T F P}: S \rightarrow \mathbb{R}_{+}$is given by

$$
T F P(s) \equiv \widetilde{A}(\mathbf{z}(s)) \widetilde{A}(\boldsymbol{\psi}(s)) \widetilde{A}(\boldsymbol{\phi}(s)) \widetilde{\Lambda}(s)^{-1} \exp \left\{-\mathbf{d}^{\prime} \log \widetilde{\Omega}(s)\right\}
$$

where for any arbitrary column vector $\boldsymbol{w}=\left(w_{1}, \ldots, w_{N}\right)^{\prime}$ of length $N$, the function $\widetilde{A}: \mathbb{R}^{N} \rightarrow \mathbb{R}$ is a log-linear operator defined as

$$
\log \widetilde{A}(\mathbf{w}) \equiv \mathbf{v}^{\prime} \widetilde{\mathbf{B}} \operatorname{diag}(\eta) \log \mathbf{w}
$$

where $\widetilde{\mathbf{B}}$ is the decreasing returns-to-scale adjusted Leontief inverse matrix given in (129) 
As mentioned, this is the the DRS analogue of Theorem 1. The only new term here is the $\exp \left\{-\mathbf{d}^{\prime} \log \widetilde{\Omega}(s)\right\}$ term in TFP. This term originates in an indirect price effect from the decreasing returns-to-scale of production. As one increases the sectoral distortions, firms operate at a lower scale (given their productivity). Operating at a lower scale implies greater marginal returns to production from any additional inputs and thereby leads to a marginal decrease in prices. Moreover a lower price index leads to greater output. Thus, greater sectoral distortions (a decrease in $\phi$ ) indirectly leads to an increase in output due to this price effect.

Finally, we solve for equilibrium consumption (GDP) and labor with homothetic preferences.

Lemma 7. Suppose utility of consumption and labor is homothetic and given by (35). Then, equilibrium aggregate labor and output (GDP) are given by the following functions, respectively:

$$
\begin{aligned}
\log L(s)= & \frac{1-\gamma}{1+\epsilon-\widetilde{\eta}(1-\gamma)} \log (\widetilde{A}(\mathbf{z}(s)) \widetilde{A}(\boldsymbol{\psi}(s)) \widetilde{A}(\phi(s)))+\frac{\gamma}{1+\epsilon-\widetilde{\eta}(1-\gamma)} \log \widetilde{\Lambda}(s) \\
& -\frac{1-\gamma}{1+\epsilon-\widetilde{\eta}(1-\gamma)}\left(\mathbf{d}^{\prime} \log \widetilde{\Omega}(s)\right) \\
\log C(s)= & \frac{1+\epsilon}{1+\epsilon-\widetilde{\eta}(1-\gamma)} \log (\widetilde{A}(\mathbf{z}(s)) \widetilde{A}(\boldsymbol{\psi}(s)) \widetilde{A}(\phi(s)))-\frac{1+\epsilon-\widetilde{\eta}}{1+\epsilon-\widetilde{\eta}(1-\gamma)} \log \widetilde{\Lambda}(s) \\
& -\frac{1+\epsilon}{1+\epsilon-\widetilde{\eta}(1-\gamma)}\left(\mathbf{d}^{\prime} \log \widetilde{\Omega}(s)\right)
\end{aligned}
$$

Lemma 7 is thereby the DRS analogue of Lemma 4 in the CRS economy.

\section{DRS Economy Proofs}

Proof of Lemma 5. The proof of this Proposition follows the same structure as the proof of Proposition 7 . We let $\mathbf{m}(s) \equiv \operatorname{diag}(\mathbf{p}(s)) \mathbf{y}(s)$ denote the equilibrium vector of sales. From market clearing, total sales for each sector must satisfy

$$
p_{i}(s) q_{i}(s)=p_{i}(s) c_{i}(s)+p_{i}(s) \sum_{j \in I} x_{j i}(s), \forall i \in I
$$

We again let $b_{i}(s)$ denote total expenditures in sector $i$ :

$$
b_{i}(s)=w_{i}(s) \ell_{i}(s)+\sum_{j} p_{j}(s) x_{i j}(s)
$$

As in the proof of Proposition 7, the Cobb-Douglas structure of preferences and technologies continues to imply constant expenditure shares of firms and households over the commodities; that is, conditions (90) and (91) continue to hold. Thus combining these conditions with the market 
clearing condition in (137) yields the following equation

$$
m_{i}(s)=v_{i} u_{0}(s)+\sum_{j \in I}\left(1-\alpha_{j}\right) g_{j i} b_{j}(s), \text { for all } i,
$$

where $m_{i}(s)=p_{i}(s) y_{i}(s)$. We stack equation (138) for each sector $i$ atop one another, and write it as the following system of equations:s

$$
\mathbf{m}(s)=\mathbf{v} u_{0}(s)+(\operatorname{diag}(\mathbf{1}-\alpha) \mathbf{G})^{\prime} \mathbf{b}(s),
$$

We htus relate total revenue of each firm to the total expenditures of each sector and the total expenditure of the household, $u_{0}(s)$.

Again as in the proof of Proposition 7 household expenditure may be written as (95). That is, the household's non-labor income is equal to the difference between total expenditures and total sales. We rewrite this in vector form as in (96). Using (96) to substitute for $u_{0}(s)$ into our market clearing condition (139), we obtain the following fixed point in sectoral sales and sectoral expenditure:

$$
\mathbf{m}(s)=\mathbf{v} L(s)+\mathbf{v} \mathbf{1}^{\prime}(\mathbf{m}(s)-\mathbf{b}(s))+(\operatorname{diag}(\mathbf{1}-\alpha) \mathbf{G})^{\prime} \mathbf{b}(s) .
$$

From firm optimality, we have that firm expenditures satisfy $b_{i}(s)=\eta_{i} \phi_{i}(s) m_{i}(s)$. This is unlike the proof of Proposition 7 due to DRS. Stacking this equation atop one another sector-bysector, we have

$$
\mathbf{b}(s)=\operatorname{diag}(\eta) \operatorname{diag}(\phi(s)) \mathbf{m}(s) \text {. }
$$

Substituting (141) for $\mathbf{b}(s)$ into (140), yields

$$
\mathbf{m}(s)=\mathbf{v} L(s)+\mathbf{v} \mathbf{1}^{\prime}(\mathbf{m}(s)-\operatorname{diag}(\eta) \operatorname{diag}(\phi(s)) \mathbf{m}(s))+(\operatorname{diag}(\mathbf{1}-\alpha) \mathbf{G})^{\prime} \operatorname{diag}(\eta) \operatorname{diag}(\phi(s)) \mathbf{m}(s)
$$

With some algebraic manipulation we may re-write this as

$$
\mathbf{m}(s)=\left[\mathbf{v} \mathbf{1}^{\prime}\left(\mathbb{I}_{N}-\operatorname{diag}(\eta) \operatorname{diag}(\phi(s))\right)+(\operatorname{diag}(\mathbf{1}-\alpha) \mathbf{G})^{\prime} \operatorname{diag}(\eta) \operatorname{diag}(\phi(s))\right] \mathbf{m}(s)+\mathbf{v} L(s)
$$

Total revenue thereby satisfies

$$
\mathbf{m}(s)=\left[\mathbb{I}_{N}-(\operatorname{diag}(\mathbf{1}-\alpha) \mathbf{G})^{\prime} \operatorname{diag}(\eta) \operatorname{diag}(\phi(s))-\mathbf{v} \mathbf{1}^{\prime}\left(\mathbb{I}_{N}-\operatorname{diag}(\eta) \operatorname{diag}(\phi(s))\right)\right]^{-1} \mathbf{v} L(s)
$$

This equation gives us (40), but with the vector $\Omega(\phi)$ as defined in (127). QED. 
Proof of Lemma 6. We now solve for equilibrium prices. Substituting (90) into (125), we have that total sales must satisfy

$$
m_{i}(s)=p_{i}(s)\left[b_{i}(s) z_{i}(s)\left(\alpha_{i}\right)^{\alpha_{i}}\left(\left(1-\alpha_{i}\right) \prod_{j=1}^{N}\left(\frac{g_{i j}}{p_{j}(s)}\right)^{g_{i j}}\right)^{1-\alpha_{i}}\right]^{\eta_{i}} .
$$

Taking logs of both sides of this equation results in the following expression

$$
\begin{aligned}
\log m_{i}(s)= & \log p_{i}(s)+\eta_{i} \log z_{i}(s)+\eta_{i} \log b_{i}(s) \\
& +\eta_{i}\left[\alpha_{i} \log \alpha_{i}+\left(1-\alpha_{i}\right) \log \left(1-\alpha_{i}\right)+\left(1-\alpha_{i}\right) \sum_{j=1}^{N} g_{i j}\left(\log g_{i j}-\log p_{j}(s)\right)\right]
\end{aligned}
$$

Therefore

$$
\log m_{i}(s)=\log p_{i}(s)+\eta_{i} \log z_{i}(s)+\eta_{i} \log b_{i}(s)+\eta_{i}\left[\kappa_{i}-\left(1-\alpha_{i}\right) \sum_{j=1}^{N} g_{i j} \log p_{j}(s)\right]
$$

where $\kappa_{i}$ is a constant for each sector given by,

$$
\kappa_{i} \equiv \alpha_{i} \log \alpha_{i}+\left(1-\alpha_{i}\right) \log \left(1-\alpha_{i}\right)+\left(1-\alpha_{i}\right) \sum_{j=1}^{N} g_{i j} \log g_{i j}
$$

Stacking equation (142) atop one another sector-by-sector, we get

$$
\begin{aligned}
{\left[\begin{array}{c}
\log m_{1}(s) \\
\log m_{2}(s) \\
\vdots \\
\log m_{N}(s)
\end{array}\right]=} & {\left[\begin{array}{c}
\log p_{1}(s) \\
\log p_{2}(s) \\
\vdots \\
\log p_{N}(s)
\end{array}\right]+\left[\begin{array}{c}
\eta_{1} \\
\eta_{2} \\
\vdots \\
\eta_{N}
\end{array}\right] \circ\left(\left[\begin{array}{c}
\log z_{1}(s) \\
\log z_{2}(s) \\
\vdots \\
\log z_{N}(s)
\end{array}\right]+\left[\begin{array}{c}
\log b_{1}(s) \\
\log b_{2}(s) \\
\vdots \\
\log b_{N}(s)
\end{array}\right]+\left[\begin{array}{c}
\kappa_{1} \\
\kappa_{2} \\
\vdots \\
\kappa_{N}
\end{array}\right]\right) } \\
& -\left[\begin{array}{c}
\eta_{1} \\
\eta_{2} \\
\vdots \\
\eta_{N}
\end{array}\right] \circ\left[\begin{array}{c}
1-\alpha_{1} \\
1-\alpha_{2} \\
\vdots \\
1-\alpha_{N}
\end{array}\right] \circ\left(\left[\begin{array}{cccc}
g_{11} & g_{12} & \cdots & g_{1 N} \\
g_{21} & g_{22} & \\
\vdots & & \ddots & \\
g_{N 1} & & & g_{N N}
\end{array}\right]\left[\begin{array}{c}
\log p_{1} \\
\log p_{2} \\
\vdots \\
\log p_{N}
\end{array}\right]\right),
\end{aligned}
$$

which we may re-write in vector form as

$$
\log \mathbf{m}(s)=\log \mathbf{p}(s)+\operatorname{diag}(\eta)(\log \mathbf{z}(s)+\log \mathbf{b}(s)+\kappa)-\operatorname{diag}(\eta) \operatorname{diag}(\mathbf{1}-\alpha) \mathbf{G} \log \mathbf{p}(s)
$$

where $\kappa \equiv\left(\kappa_{1}, \ldots, \kappa_{N}\right)^{\prime}$. We use this expression to solve for the vector of log prices as follows: 


$$
\log \mathbf{p}(s)-\operatorname{diag}(\eta) \operatorname{diag}(\mathbf{1}-\alpha) \mathbf{G} \log \mathbf{p}(s)=\log \mathbf{m}(s)-\operatorname{diag}(\eta)(\log \mathbf{z}(s)+\log \mathbf{b}(s)+\kappa)
$$

Next, taking logs of equation (141) we have

$$
\log \mathbf{b}(s)=\log \eta+\log \phi(s)+\log \mathbf{m}(s)
$$

Substituting this in for $\log$ expenditures, $\log \mathbf{b}(s)$, in the above equation yields

$\log \mathbf{p}(s)-\operatorname{diag}(\eta) \operatorname{diag}(\mathbf{1}-\alpha) \mathbf{G} \log \mathbf{p}(s)=\log \mathbf{m}(s)-\operatorname{diag}(\eta)(\log \mathbf{z}(s)+\log \eta+\log \phi(s)+\log \mathbf{m}(s)+\kappa)$

which may be written as

$\left[\mathbb{I}_{N}-\operatorname{diag}(\eta) \operatorname{diag}(\mathbf{1}-\alpha) \mathbf{G}\right] \log \mathbf{p}(s)=\left[\mathbb{I}_{N}-\operatorname{diag}(\eta)\right] \log \mathbf{m}(s)-\operatorname{diag}(\eta)(\log \mathbf{z}(s)+\log \phi(s)+\log \eta+\kappa)$

Solving this for the vector of log prices produces the following result,

$$
\begin{aligned}
\log \mathbf{p}(s)= & -\left[\mathbb{I}_{N}-\operatorname{diag}(\eta) \operatorname{diag}(\mathbf{1}-\alpha) \mathbf{G}\right]^{-1}\{\operatorname{diag}(\eta)(\log \mathbf{z}(s)+\log \phi(s)+\log \eta+\kappa)\} \\
& +\left[\mathbb{I}_{N}-\operatorname{diag}(\eta) \operatorname{diag}(\mathbf{1}-\alpha) \mathbf{G}\right]^{-1}\left[\mathbb{I}_{N}-\operatorname{diag}(\eta)\right] \log \mathbf{m}(s)
\end{aligned}
$$

as in (128). QED.

Proof of Theorem 3. With the vector of equilibrium prices, we solve for GDP, or real consumption as follows:

$$
G D P=C(s)=\prod_{j=1}^{N}\left(\frac{c_{j}(s)}{v_{j}}\right)^{v_{j}}
$$

Writing this in logs,

$$
\log C(s)=\sum_{j=1}^{N} v_{j} \log c_{j}(s)-\sum_{j=1}^{N} v_{j} \log v_{j} .
$$

Using the fact that $p_{j}(s) c_{j}(s)=v_{j} u_{0}(s)$, we get that

$$
\log C(s)=\sum_{j=1}^{N} v_{j} \log \left(\frac{v_{j} u_{0}(s)}{p_{j}(s)}\right)-\sum_{j=1}^{N} v_{j} \log v_{j}
$$

Therefore

$$
\log C(s)=\log u_{0}(s)-\sum_{j=1}^{N} v_{j} \log p_{j}(s) .
$$


We may rewrite this in vector form as

$$
\log C(s)=\log u_{0}(s)-\mathbf{v}^{\prime} \log \mathbf{p}(s)
$$

where we have used the fact that $\mathbf{v}^{\prime} \mathbf{1}=1$.

The vector of sectoral prices is given by (128). Substituting this into equation (144) gives us

$$
\begin{aligned}
\log C(s)= & \mathbf{v}^{\prime} \widetilde{\mathbf{B}} \operatorname{diag}(\eta)(\log \mathbf{z}(s)+\log \phi(s)+\log \eta+\kappa) \\
& +\log u_{0}(s)-\mathbf{v}^{\prime} \widetilde{\mathbf{B}}\left[\mathbb{I}_{N}-\operatorname{diag}(\eta)\right] \log \mathbf{m}(s)
\end{aligned}
$$

Next consider household wealth in (96). We have that it is given by

$$
u_{0}(s)=L(s)+\mathbf{1}^{\prime}(\mathbf{m}(s)-\mathbf{b}(s))
$$

combining this with (143) gives us

$$
u_{0}(s)=L(s)+\mathbf{1}^{\prime}(\mathbf{m}(s)-\operatorname{diag}(\eta) \operatorname{diag}(\phi(s)) \mathbf{m}(s))
$$

which we may write as

$$
u_{0}(s)=L(s)+\mathbf{1}^{\prime}\left(\mathbb{I}_{N}-\operatorname{diag}(\eta) \operatorname{diag}(\phi(s))\right) \mathbf{m}(s)
$$

Finally, from equation (126) in Proposition 5, we may write this as

$$
u_{0}(s)=L(s)+\mathbf{1}^{\prime}\left(\mathbb{I}_{N}-\operatorname{diag}(\eta) \operatorname{diag}(\phi(s))\right) \widetilde{\Omega}(s) L(s)
$$

Therefore household expenditure is also linear in labor and we may write this as

$$
u_{0}(s)=\widetilde{\Lambda}(s)^{-1} L(s)
$$

where

$$
\widetilde{\Lambda}(s) \equiv\left[1+\mathbf{1}^{\prime}\left(\mathbb{I}_{N}-\operatorname{diag}(\eta) \operatorname{diag}(\phi(s))\right) \widetilde{\Omega}(s)\right]^{-1}
$$

Taking logs of (5) and (146) gives us

$$
\begin{aligned}
& \log u_{0}(s)=-\log \widetilde{\Lambda}(s)+\log L(s) \\
& \log \mathbf{m}(s)=\log \widetilde{\Omega}(s)+\mathbf{1} \log L(s)
\end{aligned}
$$


Substituting these expressions into (145) we obtain

$$
\begin{aligned}
\log C(s)= & \mathbf{v}^{\prime} \widetilde{\mathbf{B}} \operatorname{diag}(\eta)(\log \mathbf{z}(s)+\log \phi(s)+\log \eta+\kappa) \\
& -\log \widetilde{\Lambda}(s)+\log L(s)-\mathbf{d}^{\prime}(\log \widetilde{\Omega}(s)+\mathbf{1} \log L(s))
\end{aligned}
$$

where $\mathbf{d}^{\prime}$ is a vector of constants defined as $\mathbf{d}^{\prime} \equiv \mathbf{v}^{\prime} \widetilde{\mathbf{B}}\left[\mathbb{I}_{N}-\operatorname{diag}(\eta)\right]$.

Collecting terms, we obtain the following expression for log GDP:

$$
\begin{aligned}
\log C(s)= & \mathbf{v}^{\prime} \widetilde{\mathbf{B}} \operatorname{diag}(\eta)(\log \mathbf{z}(s)+\log \phi(s))-\log \widetilde{\Lambda}(s) \\
& -\mathbf{d}^{\prime} \log \widetilde{\Omega}(s)+\left(1-\mathbf{d}^{\prime} \mathbf{1}\right) \log L(s)+K
\end{aligned}
$$

where $K \equiv \mathbf{v}^{\prime} \widetilde{\mathbf{B}} \operatorname{diag}(\eta)(\log \eta+\kappa)$ is a scalar. Therefore, by exponentiating (147), we may express the aggregate production funcion as follows

$$
C(s)=\widetilde{T F P}(s) L(s)^{\widetilde{\eta}},
$$

where

$$
\widetilde{\eta} \equiv 1-\mathbf{d}^{\prime} \mathbf{1}
$$

denotes the aggregate decreasing returns to scale in labor and where total factor productivity is given by

$$
T F P(s) \equiv \widetilde{A}(\mathbf{z}(s)) \widetilde{A}(\boldsymbol{\psi}(s)) \widetilde{A}(\boldsymbol{\phi}(s)) \widetilde{\Lambda}(s)^{-1} \exp \left\{-\mathbf{d}^{\prime} \log \widetilde{\Omega}(s)\right\}
$$

where the function $\widetilde{A}$ is as defined in (134) and we abstract from the constant $e^{K}$.

Finally we solve for the equilibrium aggregate labor wedge. First, note that from the houshold's budget constraint we have that aggregate consumption satisfies

$$
P(s) C(s)=u_{0}(s)=\widetilde{\Lambda}(s)^{-1} L(s)
$$

where the aggregate price index is given by

$$
\log P(s)=\mathbf{v}^{\prime} \log \mathbf{p}(s)
$$

We may rewrite (148) as

$$
\widetilde{\Lambda}(s) \frac{C(s)}{L(s)}=\frac{1}{P(s)} .
$$

Combining (150) with the household's optimality condition between consumption and labor 
yields

$$
\widetilde{\Lambda}(s) \frac{C(s)}{L(s)}=\frac{V^{\prime}(L(s))}{U^{\prime}(C(s))} .
$$

Note that given the production function in (130) exhibits decreasing returns, the aggregate marginal product of labor is now given by

$$
M P_{L}(s)=\widetilde{\eta} \frac{C(s)}{L(s)} .
$$

Using this we may rewrite condition (151) as

$$
\frac{\widetilde{\Lambda}(s)}{\widetilde{\eta}} M P_{L}(s)=\frac{V^{\prime}(L(s))}{U^{\prime}(C(s))}
$$

as in (131). QED.

Proof of Lemma 7. With the homothetic preferences stated in (35), we have the following system of two equations and two unknowns:

$$
\begin{aligned}
L(s)^{\epsilon} & =C(s)^{-\gamma} \widetilde{\Lambda}(s) \frac{C(s)}{L(s)} \\
C(s) & =\widetilde{A}(\mathbf{z}(s)) \widetilde{A}(\boldsymbol{\psi}(s)) \widetilde{A}(\phi(s)) \exp \left\{-\mathbf{d}^{\prime} \log \widetilde{\Omega}(s)\right\} \widetilde{\Lambda}(s)^{-1} L(s)^{\widetilde{\eta}}
\end{aligned}
$$

Substituting expression (153) for consumption into (152), we get

$$
L(s)^{1+\epsilon}=\widetilde{\Lambda}(s)\left(\widetilde{A}(\mathbf{z}(s)) \widetilde{A}(\boldsymbol{\psi}(s)) \widetilde{A}(\boldsymbol{\phi}(s)) \exp \left\{-\mathbf{d}^{\prime} \log \widetilde{\Omega}(s)\right\} \widetilde{\Lambda}(s)^{-1} L(s)^{\widetilde{\eta}}\right)^{1-\gamma}
$$

Solving this expression for $L(s)$,

$$
L(s)^{1+\epsilon-\widetilde{\eta}(1-\gamma)}=\widetilde{\Lambda}(s)^{\gamma}\left(\widetilde{A}(\mathbf{z}(s)) \widetilde{A}(\boldsymbol{\psi}(s)) \widetilde{A}(\boldsymbol{\phi}(s)) \exp \left\{-\mathbf{d}^{\prime} \log \widetilde{\Omega}(s)\right\}\right)^{1-\gamma},
$$

and taking logs of both sides gives us equilibrium aggregate labor

$$
\begin{aligned}
\log L(s)= & \frac{1-\gamma}{1+\epsilon-\widetilde{\eta}(1-\gamma)} \log (\widetilde{A}(\mathbf{z}(s)) \widetilde{A}(\boldsymbol{\psi}(s)) \widetilde{A}(\phi(s)))+\frac{\gamma}{1+\epsilon-\widetilde{\eta}(1-\gamma)} \log \widetilde{\Lambda}(s) \\
& -\frac{1-\gamma}{1+\epsilon-\widetilde{\eta}(1-\gamma)}\left(\mathbf{d}^{\prime} \log \widetilde{\Omega}(s)\right)
\end{aligned}
$$


as in (135). Finally, substituting this back into the aggregate production function (153) yields the following expression for equilibrium aggregate consumption

$$
\begin{aligned}
\log C(s)= & \frac{1+\epsilon}{1+\epsilon-\widetilde{\eta}(1-\gamma)} \log (\widetilde{A}(\mathbf{z}(s)) \widetilde{A}(\boldsymbol{\psi}(s)) \widetilde{A}(\phi(s)))-\frac{1+\epsilon-\widetilde{\eta}}{1+\epsilon-\widetilde{\eta}(1-\gamma)} \log \widetilde{\Lambda}(s) \\
& -\frac{1+\epsilon}{1+\epsilon-\widetilde{\eta}(1-\gamma)}\left(\mathbf{d}^{\prime} \log \widetilde{\Omega}(s)\right)
\end{aligned}
$$

as in (136). QED. 